\title{
Systematics of the Osteocephalus buckleyi species complex (Anura, Hylidae) from Ecuador and Peru
}

\author{
Santiago R. Ron ${ }^{1, \dagger}$, Pablo J. Venegas ${ }^{2, \ddagger}$, Eduardo Toral ${ }^{1,3, \S}$, Morley Read ${ }^{1,1}$, \\ Diego A. Ortiz ${ }^{1, \pi}$, Andrea L. Manzano ${ }^{1,4, \#}$
}

I Museo de Zoología, Escuela de Biología, Pontificia Universidad Católica del Ecuador, Av. 12 de Octubre y Roca, Aptdo. 17-01-2184, Quito, Ecuador 2 División de Herpetología-Centro de Ornitología y Biodiversidad (CORBIDI), Santa Rita N¹05 Of. 202, Urb. Huertos de San Antonio, Surco, Lima, Perú 3 Current address: Facultad de Ciencias Ambientales, Universidad Internacional SEK, Quito, Ecuador 4 Current address: Biology Department, HH227, San Francisco State University, 1600 Holloway Avenue, San Francisco, CA 94132, USA

† urn:lsid:zoobank.org:author:ACF9C463-F771-459C-B22B-AF6B9902DF57

¥ urn:lsid:zoobank.org:author:15AD03E1-9ACF-4F38-AA96-09A5A56A3DC4

§ urn:lsid:zoobank.org:author:1A4F31AF-5629-4EE5-83DD-1EBB93139A5B

| urn:lsid:zoobank.org:author:10D75453-75A0-49C7-B577-A7687398CEFO

I urn:lsid:zoobank.org:author:51147772-F315-412E-BA89-B8990CA49544

\# urn:lsid:zoobank.org:author:7C29ADA6-00FB-45DC-9A5B-7B9A217B71F6

Corresponding author: Santiago R. Ron (santiago.r.ron@gmail.com)

Academic editor: F. Andreone | Received 24 June 2012 | Accepted 5 October 2012 | Published 18 October 2012

urn:lsid:zoobank.org:pub:163872F0-BE66-436C-9663-EE2226C358AB

Citation: Ron SR, Venegas PJ, Toral E, Read M, Ortiz DA, Manzano AL (2012) Systematics of the Osteocephalus buckleyi species complex (Anura, Hylidae) from Ecuador and Peru. ZooKeys 229: 1-52. doi: 10.3897/zookeys.229.3580

\begin{abstract}
We present a new phylogeny, based on DNA sequences of mitochondrial and nuclear genes, for frogs of the genus Osteocephalus with emphasis in the Osteocephalus buckleyi species complex. Genetic, morphologic, and advertisement call data are combined to define species boundaries and describe new species. The phylogeny shows strong support for: (1) a basal position of $O$. taurinus + O. oophagus, (2) a clade containing phytotelmata breeding species, and (3) a clade that corresponds to the $O$. buckleyi species complex. Our results document a large proportion of hidden diversity within a set of populations that were previously treated as a single, widely distributed species, $O$. buckleyi. Individuals assignable to $O$. buckleyi formed a paraphyletic group relative to $O$. verruciger and $O$. cabrerai and contained four species, one of which is $O$. buckleyi sensu stricto and three are new. Two of the new species are shared between Ecuador and Peru (O. vilmae sp. n. and O. cannatellai sp. n.) and one is distributed in the Amazon region of southern Peru (O. germani sp. n.). We discuss the difficulties of using morphological characters to define species boundaries and propose a hypothesis to explain them.
\end{abstract}

Copyright Santiago R. Ron et al. This is an open access article distributed under the terms of the Creative Commons Attribution License 3.0 (CC-BY), which permits unrestricted use, distribution, and reproduction in any medium, provided the original author and source are credited. 


\section{Resumen}

Presentamos una nueva filogenia, basada en secuencias de ADN de genes nucleares y mitocondriales, para ranas del género Osteocephalus con énfasis en el complejo de especies Osteocephalus buckleyi. Datos genéticos, morfológicos, y de cantos de anuncio se combinan para definir límites de especies y describir nuevas especies. La filogenia muestra un soporte fuerte para: (1) una posición basal de $O$. taurinus $+O$. oophagus, (2) un clado que contiene especies con reproducción en fitotelmatas, y (3) un clado que corresponde el complejo de especies $O$. buckleyi. Nuestros resultados documentan una gran proporción de diversidad escondida dentro de un grupo de poblaciones que previamente habían sido tratadas como una sola especie ampliamente distribuida, $O$. buckleyi. Los individuos asignables a $O$. buckleyi formaron un grupo parafilético en relación a $O$. verruciger y $O$. cabrerai y contuvieron cuatro especies, una de las cuales es $O$. buckleyi sensu scricto y tres son nuevas. Dos de las nuevas especies están compartidas entre Ecuador y Perú (O. vilmae sp. n. y O. cannatellai sp. n.) y una está en el sur de Perú (O. germani sp. n.) Discutimos las dificultades de usar caracteres morfológicos para definir límites de especies y proponemos una hipótesis para explicarla.

\section{Keywords}

Advertisement calls, Amazon, Anura, Cryptic species, Morphology, Osteocephalus buckleyi, Phylogeny

\section{Introduction}

The Upper Amazon region has the highest alpha diversity of amphibians in the World with several sites exceeding 100 species in less than $10 \mathrm{~km}^{2}$ (Bass et al. 2010). Remarkably, these figures may vastly underestimate the total diversity as shown by the discovery of large numbers of cryptic species with the use of genetic markers (e.g., Fouquet et al. 2007; Funk et al. 2011; Padial and De la Riva 2009; Ron et al. 2006). These preliminary efforts suggest that the use of genetic characters is crucial to attain a complete understanding of the diversity and evolutionary history of Amazonian amphibians. This necessity is particularly pressing in widespread taxa with pervasive taxonomic problems.

One such group is Osteocephalus, a genus of hylid frogs widely distributed in the Amazon Basin, Guianas and upper drainages of the Magdalena and Orinoco rivers (Frost 2010). Osteocephalus are arboreal and nocturnal frogs with reproduction modes varying from deposition of eggs in lentic water and exotrophic tadpoles to deposition of eggs in bromeliads and oophagus tadpoles and biparental care (Crump 1974; Jungfer and Weygoldt 1999). There are 24 described species and reports of undescribed species are frequent (e.g., Jungfer 2010; Moravec et al. 2009; Ron et al. 2010). There is only one formally defined species group within Osteocephalus, the O. buckleyi complex. It was first proposed by Cochran and Goin (1970) to allocate O. buckleyi (Boulenger 1882), O. pearsoni (Gaige 1929), and O. cabrerai (Cochran and Goin 1970). Its first large scale review was carried out by Trueb and Duellman (1971) who examined the morphology of specimens from seven countries and concluded that the $O$. buckleyi complex (excluding O. verruciger Werner 1901) consisted of a single, morphologi- 
cally variable and widely distributed species. They synonymized $O$. cabrerai, O. carri (Cochran and Goin 1970), and O. festae (Peracca 1904) under O. buckleyi. The three species have been subsequently resurrected (Duellman and Mendelson 1995; Jungfer 2010; Lynch 2006). Recent reviews (Jungfer 2010; 2011; Moravec et al. 2009; Ron et al. 2010) imply that the $O$. buckleyi species complex consists of nine species: $O$. buckleyi, O. cabrerai, O. carri, O. duellmani Jungfer 2011, O. festae, O. inframaculatus (Boulenger 1882), O. mutabor Jungfer and Hödl 2002, O. verruciger and an undescribed species sister to $O$. verruciger. A phylogeny based on mitochondrial DNA revealed strong support for the $O$. buckleyi complex as well as paraphyly in $O$. verruciger and O. buckleyi (Ron et al. 2010).

Despite recent contributions to the taxonomy of the group (e.g., Jungfer 2010; 2011) the O. buckleyi species complex still contains undescribed species as well as alpha taxonomic problems (Jungfer 2010; Ron et al. 2010) which attest the difficulties of correctly identifying species boundaries on the basis of morphological evidence alone. Herein we integrate genetic, morphological and advertisement call data to assess the phylogenetic relationships and species boundaries among populations of the $O$. buckleyi complex from Ecuador and Peru. The results demonstrate the existence of three new species, which are formally described here.

\section{Methods}

For ease of comparison, we generally follow the format of Trueb and Duellman (1971) for diagnosis and description. Morphological terminology and abbreviations follow Lynch and Duellman (1997). Notation for hand and foot webbing is based on Myers and Duellman (1982). Sex was determined by the texture of dorsal skin, the presence of nuptial pads or vocal sac folds, and by gonadal inspection. Specimens were fixed in 10\% formalin and preserved in 70\% ethanol. Snout-vent length is abbreviated as SVL. Examined specimens (listed in the type-series and Appendix I) are housed at the collection of the División de Herpetología, Centro de Ornitología y Biodiversidad (CORBIDI), Herpetology Collection at Escuela Politécnica Nacional (EPN-H), Museo de Historia Natural at Universidad San Marcos (MUSM), Museo de Zoología at Pontificia Universidad Católica del Ecuador (QCAZ), and Natural History Museum (BMNH). The pencil drawing of the holotype of O. cannatella sp. n. was made using a Wild Heerbrugg M3B 10×/21 stereo microscope equipped with a camera lucida.

Principal Components Analysis (PCA) and Discriminant Function Analysis (DFA) were used to assess the degree of morphometric differentiation between species. Only well preserved specimens (Simmons 2002) were measured for the following eight morphological variables, following Duellman (1970): (1) SVL; (2) head length; (3) head width; (4) tympanum diameter; (5) femur length; (6) tibia length; (7) foot length; and (8) eye diameter. All variables were log-transformed. To remove the effect of co- 
variation with SVL, the PCA was applied to the residuals from the linear regressions between the seven measured variables and SVL. We applied a multivariate analysis of variance (MANOVA) to tests for morphometric differences between sexes. Because we found significant differences in O. buckleyi, the PCA and DFA were applied on each sex separately. For the PCA, only components with eigenvalues $>1$ were retained. The DFA was applied to the measured variables without size correction because we wanted to assess discriminability among species based on all the variables, including SVL. Sample sizes are: $O$. buckleyi 24 males, 3 females; O. cabrerai 7 males; O. cannatellai sp. n. 33 males, 3 females; $O$. festae 7 males, 18 females; $O$. germani sp. n. 2 males, 5 females; $O$. verruciger 22 males, 5 females; and $O$. vilmae sp. $\mathrm{n}$. 4 males. Both PCA and DFA were conducted in $\mathrm{JMP}^{\circ} 8.01$ (SAS Institute 2008). Measurements were made using digital calipers (to the nearest $0.01 \mathrm{~mm}$ ).

Advertisement calls recordings were made with a Sennheiser ${ }^{\mathrm{TM}}$ ME-67 directional microphone with digital recorder Olympus ${ }^{\mathrm{TM}} \mathrm{LS10}$. Calls were analyzed using software Raven 1.2.1 (Charif et al. 2004) at a sampling frequency of $22.1 \mathrm{kHz}$ and a frequency resolution of $21.5 \mathrm{~Hz}$. Calls consist of two components, the first is a rattle note and the second is a quack note. Measured call variables are: (1) call rate: number of calls per second, (2) dominant frequency: frequency with the most energy, measured along all the call, (3) duration of first component note: time from the beginning to the end of note, (4) duration of second component: time from beginning of first quack to the end of the last, (5) first component interval: time from the end of last note of the first component to the beginning of the first note of the second component, (6) number of pulses: number of pulses in a first component note, (7) pulse rate: number of pulses/ duration of first component note, (8) duration of second component note: duration from beginning to end of a single quack, (9) quack rate: number of quacks/duration of second component. If available, several calls or notes were analyzed per individual to calculate an individual average. Original recordings are deposited in the audio archive of the QCAZ and are available through the AmphibiaWebEcuador website (http://zoologia.puce.edu.ec/vertebrados/anfibios/).

\section{DNA extraction, amplification, and sequencing}

Total DNA was extracted from muscle or liver tissue preserved in 95\% ethanol or tissue storage buffer using standard phenol-chloroform extraction protocols (Sambrook et al. 1989). Polymerase chain reaction (PCR) was used to amplify the mitochondrial genes 12S rRNA ,16S rRNA, ND1 (with flanking tRNA genes), CO1, and control region. We amplified one DNA fragment for $12 \mathrm{~S}$, CO1, and the control region and one or two overlapping fragments for the last $-320 \mathrm{bp}$ of $16 \mathrm{~S}$ and the adjacent ND1 using primers listed in Goebel et al. (1999) and Moen and Wiens (2009). We also amplified the nuclear gene POMC as a single fragment using primers listed by Wiens et al. (2005). PCR amplification was carried under standard protocols. Amplified products were sequenced by the Macrogen Sequencing Team (Macrogen Inc., Seoul, Korea). 


\section{Phylogenetic analyses}

We estimated phylogenetic relations between species of Osteocephalus based on newly generated sequence data for five mitochondrial (12S RNA, CO1, 16S, ND1, control region) and one nuclear gene (POMC) for a total of up to $4170 \mathrm{bp}$. To expand the species sampling, we also included sequences from GenBank. All samples are listed in Table 1. For the outgroup, we included one sample of Trachycephalus jordani and one of T. typhonius (based on Faivovich et al. 2005 and Wiens et al. 2010). The completeness of the sequences varied considerably among individuals (specially for samples from GenBank which typically lacked three or more loci). Nevertheless, we included samples with missing data because analyses of both empirical and simulated matrices have shown that taxa with missing sequences can be accurately placed in model-based phylogenetic analyses if the number of characters is large, as in our matrix (for a review see Wiens and Morrill 2011).

Preliminary sequence alignment was done with MAFFT $6.814 \mathrm{~b}$ software with the LINS-i algorithm (Katoh et al. 2002). The sequence matrix was imported to Mesquite (version 2.72; Maddison and Maddison 2009) and the ambiguously aligned regions were adjusted manually to produce a parsimonious alignment (i.e., informative sites minimized). In protein coding loci, DNA sequences were translated to amino acids with Mesquite to aid the manual alignment. Phylogenetic trees were obtained using Bayesian inference.

Because our dataset includes several loci, it is unlikely that it fits a single model of nucleotide substitution. Thus, we partitioned the data to analyze each partition under a separate model. The best model for each partition was chosen with JModelTest version 0.1.1 (Posada 2008) using the Akaike Information Criterion with sample size correction as optimality measure. We also evaluated three different partition strategies: (i) a single partition, (ii) six partitions (one per loci), and (iii) twelve partitions (one for each codon position in protein coding loci plus one for each non protein coding loci). The best partition strategy was chosen by estimating Bayes factors using a threshold of 10 as evidence in favor of the more complex partition (Brandley et al. 2005).

Each Bayesian analysis consisted of two parallel runs of the Metropolis coupled Monte Carlo Markov chain for $5 \times 10^{6}$ generations. Each run had four chains with a temperature of 0.05 . The prior for the rate matrix was a uniform dirichlet and all topologies were equally probable a priori. Convergence into a stationary distribution was determined by reaching average standard deviation split frequencies $<0.05$ between runs. We also used software Tracer ver. 1.5 (Rambaut and Drummond 2007) to visually inspect convergence and stationarity of the runs. The first $50 \%$ of the sampled generations were discarded as burn-in and the remaining were used to estimate the Bayesian tree, posterior probabilities and other model parameters. Phylogenetic analyses were carried out in MrBayes 3.2.1 (Ronquist et al. 2012).

Because the only nuclear gene analyzed had low variability and few informative sites, it was concatenated to the mitochondrial genes into a single matrix. We recognize the advantages of species-tree methods (e.g., Edwards et al. 2007) but could not use them given the insufficient number of nuclear genes sampled. We encourage the application of those methodologies in future phylogenetic inferences in Osteocephalus. 


\begin{tabular}{|c|c|c|c|c|c|c|c|c|c|c|c|c|c|c|c|c|c|c|c|c|}
\hline 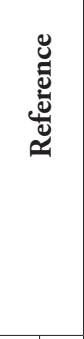 & 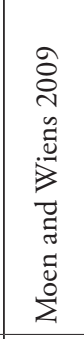 & 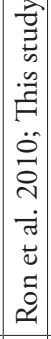 & 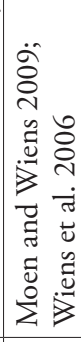 & 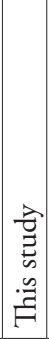 & 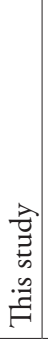 & 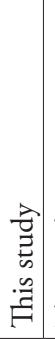 & 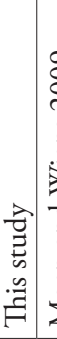 & 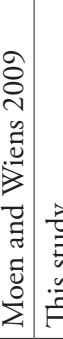 & 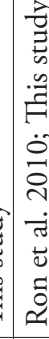 & 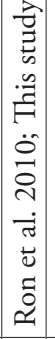 & 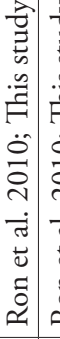 & 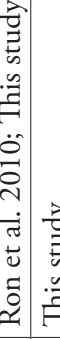 & 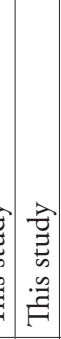 & 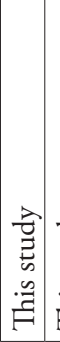 & 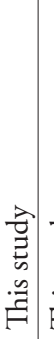 & & 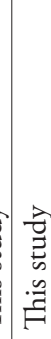 & 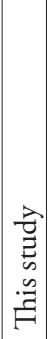 & 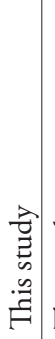 & $\vec{E}$ \\
\hline 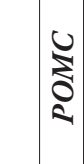 & 1 & 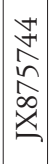 & 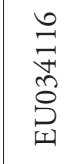 & 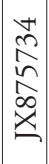 & $\begin{array}{l}\tilde{n} \\
\tilde{n} \\
\hat{\infty} \\
\underline{x}\end{array}$ & 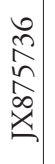 & $\begin{array}{l}\hat{n} \\
\hat{n} \\
\infty \\
\infty \\
\underline{\alpha}\end{array}$ & $\mid \begin{array}{l}\mathfrak{T} \\
\mathfrak{N} \\
\mathfrak{n} \\
\mathfrak{x} \\
\mathfrak{z}\end{array}$ & $\begin{array}{l}n \\
\tilde{n} \\
n \\
\infty \\
x \\
x\end{array}$ & 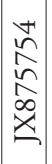 & 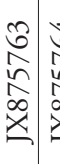 & 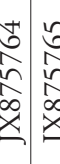 & 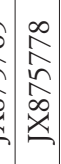 & $\begin{array}{l}\infty \\
\infty \\
n \\
n \\
\infty \\
x \\
\end{array}$ & 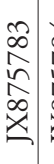 & 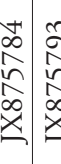 & $\begin{array}{l}\infty \\
\curvearrowright \\
\hat{n} \\
\infty \\
x \\
\underline{x}\end{array}$ & 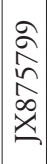 & 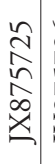 & \\
\hline 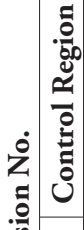 & 1 & 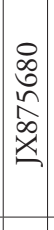 & 1 & $i$ & 1 & 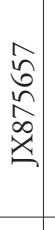 & i & $\mid \begin{array}{l}\infty \\
\hat{n} \\
\hat{n} \\
\not{x}\end{array}$ & $\begin{array}{l}\infty \\
\stackrel{2}{N} \\
\hat{n} \\
\infty \\
x \\
\end{array}$ & 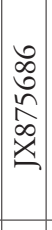 & 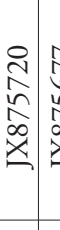 & 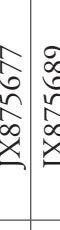 & 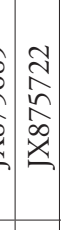 & $\begin{array}{l} \pm \\
\hat{N} \\
\hat{\infty} \\
\tilde{x} \\
\Sigma\end{array}$ & 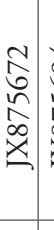 & & $\begin{array}{l}\mathcal{1} \\
\hat{n} \\
\hat{n} \\
\infty \\
x \\
\underline{x}\end{array}$ & $\mid \begin{array}{l}n \\
\hat{2} \\
\hat{n} \\
\hat{\alpha} \\
x \\
\underline{x}\end{array}$ & 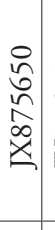 & $\begin{array}{l}\hat{0} \\
\hat{n} \\
\hat{n} \\
\infty \\
x \\
\end{array}$ \\
\hline 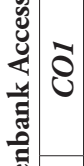 & 1 & $i$ & $i$ & 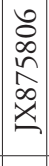 & 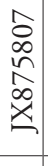 & 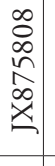 & i & $\mid \begin{array}{l}\tilde{y} \\
\infty \\
\tilde{n} \\
\infty \\
x\end{array}$ & 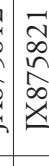 & 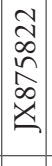 & 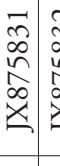 & 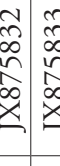 & 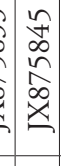 & 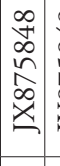 & 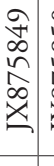 & 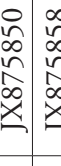 & 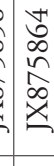 & 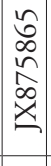 & 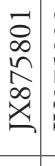 & 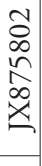 \\
\hline$\underset{N}{N}$ & i & 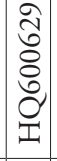 & $\begin{array}{l}\infty \\
\hat{n} \\
\stackrel{0}{ } \\
\infty \\
\stackrel{2}{0} \\
0\end{array}$ & 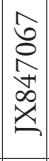 & 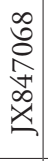 & 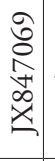 & 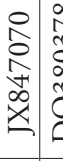 & 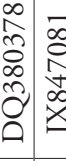 & 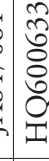 & 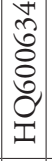 & 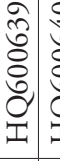 & 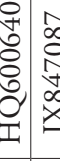 & 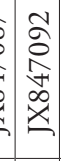 & 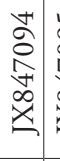 & 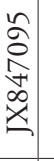 & : & 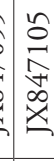 & 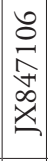 & 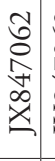 & 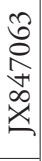 \\
\hline 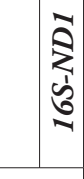 & $\begin{array}{l}\infty \\
\infty \\
\stackrel{+}{+} \\
\infty \\
\stackrel{2}{ } \\
\stackrel{1}{|c|}\end{array}$ & 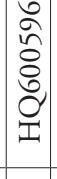 & 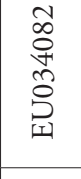 & 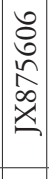 & 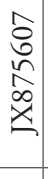 & $\begin{array}{l}\infty \\
0 \\
0 \\
\\
\infty \\
\check{c}\end{array}$ & $1 \mid$ & 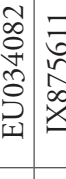 & $\begin{array}{l}8 \\
0 \\
0 \\
8 \\
0 \\
0 \\
0 \\
1\end{array}$ & 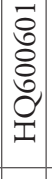 & 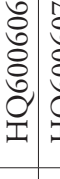 & 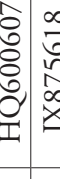 & 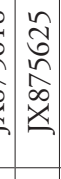 & 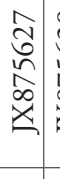 & 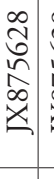 & 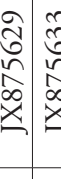 & 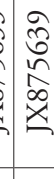 & 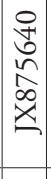 & 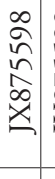 & $\begin{array}{l}\hat{n} \\
\hat{\infty} \\
x \\
\underline{x}\end{array}$ \\
\hline 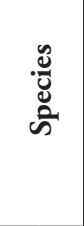 & 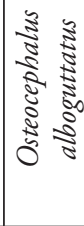 & $\left|\begin{array}{c}0 \\
3 \\
0 \\
0 \\
0 \\
0 \\
0 \\
0 \\
0 \\
0 \\
0\end{array}\right|$ & $\begin{array}{l}\frac{\pi}{3} \\
\frac{3}{3} \\
\frac{3}{0} \\
0\end{array}$ & $\mid \begin{array}{l}\tilde{z} \\
\tilde{z} \\
\tilde{z} \\
\tilde{z} \\
0 \\
0\end{array}$ & $\begin{array}{c}\frac{\pi}{3} \\
\frac{a}{3} \\
\text { בे } \\
0 \\
0\end{array}$ & 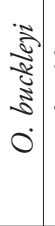 & $\begin{array}{c}0 \\
0 \\
0 \\
0 \\
0 \\
0 \\
0\end{array}$ & 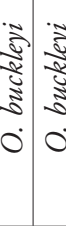 & \begin{tabular}{|c|c}
$\frac{\pi}{3}$ \\
$\frac{3}{3}$ \\
3 \\
0 \\
0
\end{tabular} & $\mid$ & $\begin{array}{c}\bar{z} \\
\frac{0}{2} \\
\frac{3}{3} \\
0 \\
0\end{array}$ & 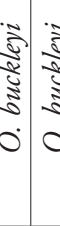 & 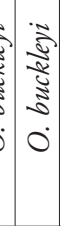 & 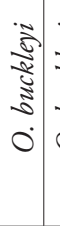 & $\begin{array}{c}0 \\
\frac{3}{2} \\
\frac{1}{3} \\
\vdots \\
0 \\
0\end{array}$ & 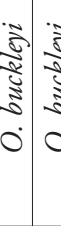 & 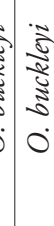 & 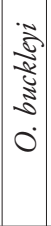 & $\begin{array}{c}0 \\
0 \\
0 \\
0 \\
0 \\
0 \\
0\end{array}$ & 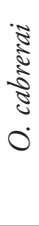 \\
\hline 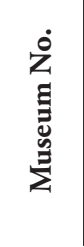 & 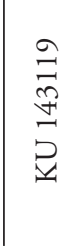 & 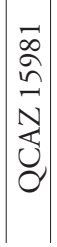 & 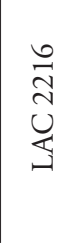 & 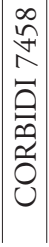 & 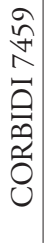 & 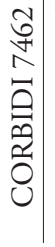 & 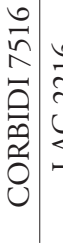 & 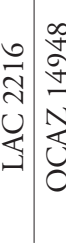 & y & 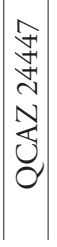 & 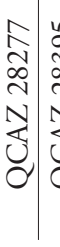 & 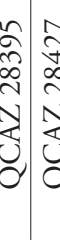 & 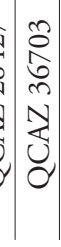 & 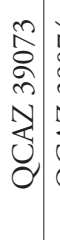 & 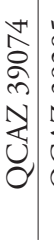 & 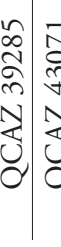 & 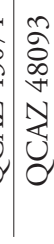 & 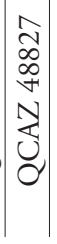 & 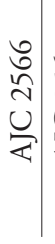 & $\begin{array}{l}\hat{\sigma} \\
\approx \\
\approx\end{array}$ \\
\hline
\end{tabular}




\begin{tabular}{|c|c|c|c|c|c|c|c|c|c|c|c|c|c|c|c|c|c|c|c|c|c|c|c|c|c|}
\hline & & 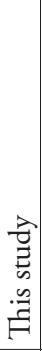 & 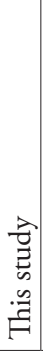 & 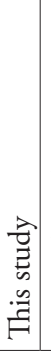 & 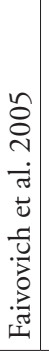 & 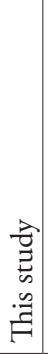 & 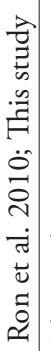 & 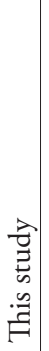 & 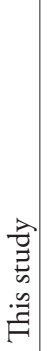 & 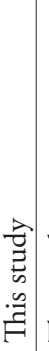 & 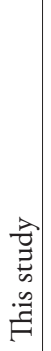 & 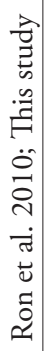 & 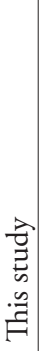 & 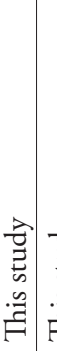 & 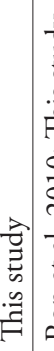 & 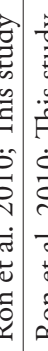 & 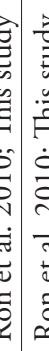 & 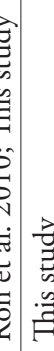 & 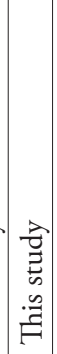 & 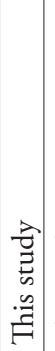 & 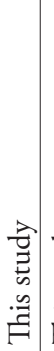 & 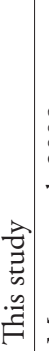 & 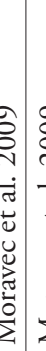 & 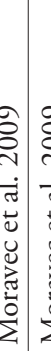 & 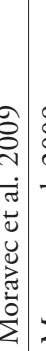 \\
\hline & 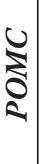 & $\begin{array}{l}\hat{N} \\
\hat{n} \\
\hat{\infty} \\
\underline{x}\end{array}$ & $\begin{array}{l}\bar{n} \\
\hat{n} \\
\hat{\infty} \\
\underline{x}\end{array}$ & $\begin{array}{l}\tilde{n} \\
\hat{n} \\
\infty \\
x \\
\varkappa\end{array}$ & $i$ & $\begin{array}{l}0 \\
\vdots \\
\hat{n} \\
\infty \\
\underline{x} \\
\underline{x}\end{array}$ & 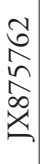 & 1 & $i$ & $i$ & 1 & $\begin{array}{l}n \\
\hat{n} \\
\hat{n} \\
\infty \\
\underline{x}\end{array}$ & $\begin{array}{l}\overrightarrow{\hat{N}} \\
\hat{n} \\
\infty \\
\underline{x}\end{array}$ & $\begin{array}{c}N \\
\hat{N} \\
\tilde{n} \\
\infty \\
x \\
z \\
z\end{array}$ & 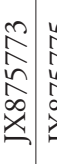 & 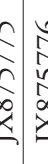 & 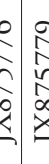 & 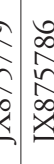 & $\begin{array}{l}\infty \\
\infty \\
\hat{n} \\
\kappa \\
\infty \\
\underline{x} \\
\underline{n}\end{array}$ & 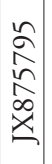 & $\begin{array}{l}\hat{\alpha} \\
\hat{\hat{n}} \\
\alpha \\
\infty \\
\underline{x}\end{array}$ & $\begin{array}{l}8 \\
8 \\
\infty \\
\\
\infty \\
x \\
\end{array}$ & i & i & i \\
\hline$\underline{\dot{z}}$ & 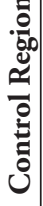 & $\begin{array}{l}\vec{n} \\
\curvearrowleft \\
\\
\infty \\
\underline{x}\end{array}$ & $\begin{array}{l}\tilde{\kappa} \\
\swarrow \\
\curvearrowleft \\
\infty \\
x\end{array}$ & 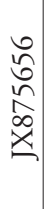 & 1 & $\begin{array}{l}\stackrel{o}{0} \\
\hat{n} \\
\infty \\
x \\
\underline{x}\end{array}$ & $\begin{array}{l}0 \\
\hat{\lambda} \\
\hat{n} \\
\infty \\
x \\
\end{array}$ & 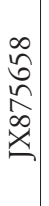 & 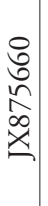 & 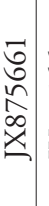 & 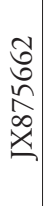 & 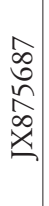 & 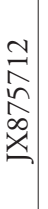 & 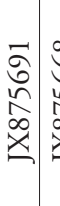 & 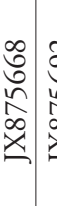 & 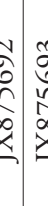 & 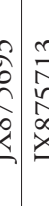 & 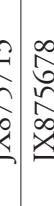 & 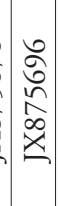 & $\begin{array}{l}\overrightarrow{2} \\
\hat{n} \\
\hat{n} \\
x \\
\underline{x}\end{array}$ & $\begin{array}{l}\hat{乏} \\
\hat{n} \\
\infty \\
x \\
\varkappa\end{array}$ & 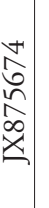 & i & i & i \\
\hline 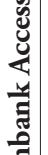 & $\widehat{ఠ}$ & 1 & $\begin{array}{l}\stackrel{+}{0} \\
\infty \\
\curvearrowleft \\
\\
\infty \\
\underline{x}\end{array}$ & 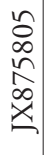 & $i$ & $\begin{array}{l}\hat{N} \\
\infty \\
\curvearrowleft \\
\infty \\
x \\
\end{array}$ & $\begin{array}{l}0 \\
\Uparrow \\
\infty \\
\curvearrowleft \\
\infty \\
\underline{x} \\
\end{array}$ & 1 & $i$ & $i$ & 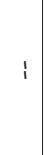 & 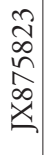 & 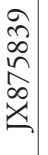 & 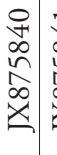 & 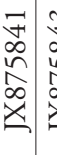 & 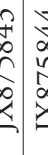 & 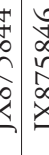 & 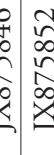 & 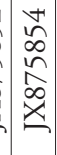 & 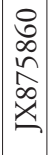 & 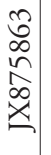 & 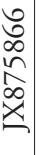 & i & i & i \\
\hline & $\underset{\mathfrak{N}}{\mathbb{N}}$ & $i$ & 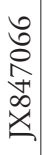 & $i$ & 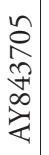 & 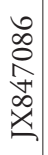 & 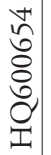 & 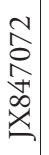 & 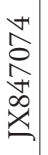 & 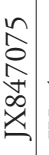 & $\begin{array}{l}\hat{N} \\
\hat{o} \\
\hat{\alpha} \\
\infty \\
\underline{x} \\
\underline{x}\end{array}$ & 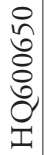 & 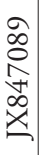 & 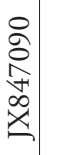 & $i$ & 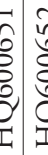 & 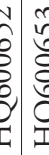 & 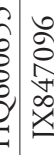 & 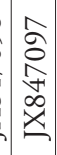 & 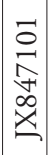 & 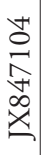 & 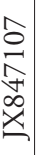 & 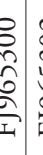 & 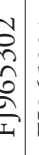 & 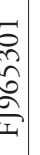 \\
\hline & 0 & $\begin{array}{l}8 \\
0 \\
\swarrow \\
\curvearrowleft \\
\infty \\
x \\
\end{array}$ & 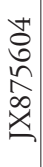 & $\begin{array}{l}n \\
b \\
\swarrow \\
n \\
\infty \\
x \\
\underline{x}\end{array}$ & 1 & $\begin{array}{l}\hat{\sigma} \\
\curvearrowleft \\
\Uparrow \\
\infty \\
\check{x}\end{array}$ & 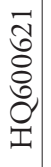 & i & 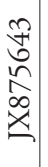 & 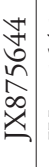 & 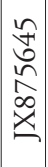 & 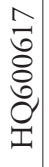 & 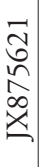 & $\begin{array}{l}\tilde{\sigma} \\
\curvearrowleft \\
\infty \\
\infty \\
\varkappa\end{array}$ & 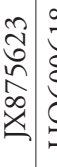 & 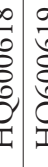 & 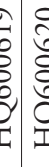 & 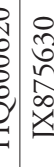 & 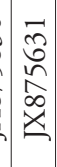 & 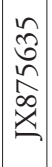 & 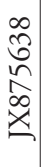 & 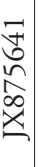 & & ; & i \\
\hline
\end{tabular}

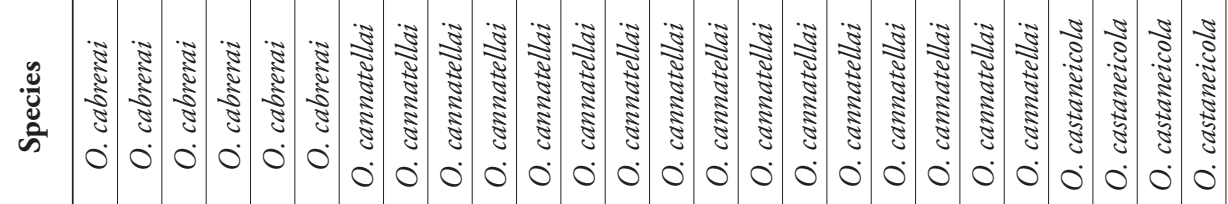

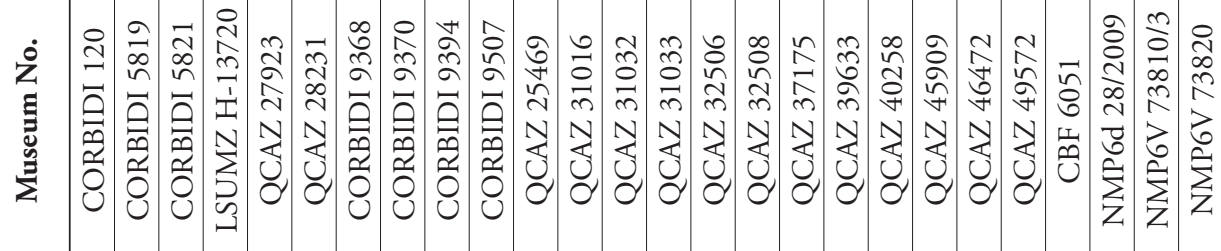




\begin{tabular}{|c|c|c|c|c|c|c|c|c|c|c|c|c|c|c|c|c|c|c|c|c|c|c|c|c|c|}
\hline & & 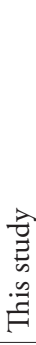 & 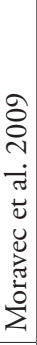 & 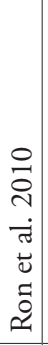 & 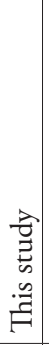 & 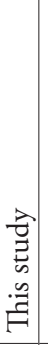 & 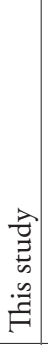 & 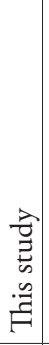 & 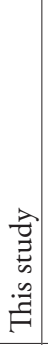 & 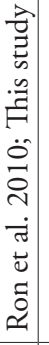 & 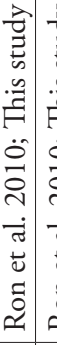 & 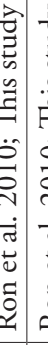 & 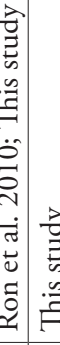 & 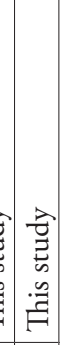 & 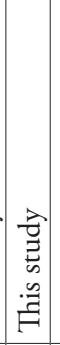 & 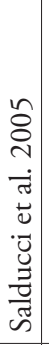 & 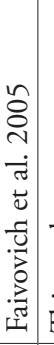 & 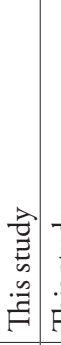 & 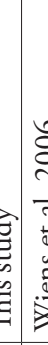 & 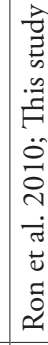 & 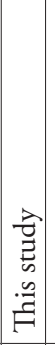 & 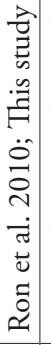 & 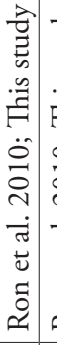 & 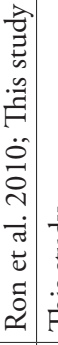 & 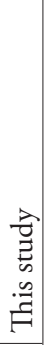 \\
\hline & వి & 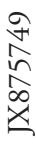 & i & $\begin{array}{l}\tilde{n} \\
\hat{n} \\
\infty \\
x \\
\end{array}$ & $\begin{array}{l}\infty \\
\tilde{n} \\
\hat{n} \\
\infty \\
\underline{x} \\
\end{array}$ & 1 & $\begin{array}{l}\stackrel{\infty}{N} \\
\hat{n} \\
\infty \\
\infty \\
\end{array}$ & $i$ & i & $\begin{array}{l}\vec{\infty} \\
\hat{n} \\
\infty \\
x \\
\underline{x}\end{array}$ & 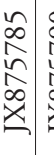 & 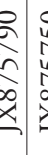 & 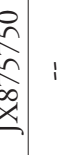 & $\begin{array}{l}\tilde{n} \\
\hat{n} \\
\infty \\
x \\
\underline{n}\end{array}$ & 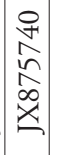 & $i$ & i & $\begin{array}{l}\stackrel{\curvearrowright}{N} \\
\hat{n} \\
\infty \\
\underline{x}\end{array}$ & i & $\begin{array}{l}\stackrel{0}{n} \\
\hat{n} \\
\infty \\
x \\
\underline{x}\end{array}$ & $\begin{array}{l}\hat{n} \\
\hat{n} \\
\hat{n} \\
\infty \\
\varkappa \\
\end{array}$ & i & 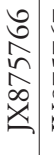 & $\begin{array}{l}\hat{\sigma} \\
\hat{n} \\
\hat{n} \\
\infty \\
x \\
\underline{x}\end{array}$ & $\begin{array}{l}\infty \\
\hat{0} \\
\hat{n} \\
\infty \\
x \\
\underline{x}\end{array}$ \\
\hline $\begin{array}{l}0 \\
z \\
z\end{array}$ & 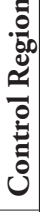 & 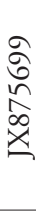 & i & $\begin{array}{l}\mathfrak{n} \\
\hat{n} \\
\hat{n} \\
x \\
\check{\varkappa}\end{array}$ & i & I & $i$ & 1 & $i$ & $i$ & 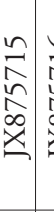 & 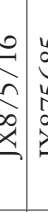 & 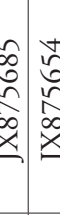 & 1 & i & i & i & 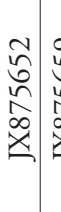 & & 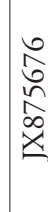 & 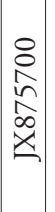 & 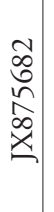 & $\begin{array}{l}\vec{N} \\
\hat{N} \\
\hat{\infty} \\
\underline{x} \\
\underline{z}\end{array}$ & $\begin{array}{l}n \\
\sigma \\
\curvearrowleft \\
\infty \\
x \\
\Sigma \\
z\end{array}$ & $\begin{array}{l}\stackrel{+}{ } \\
\hat{n} \\
\hat{n} \\
\stackrel{x}{\varkappa}\end{array}$ \\
\hline 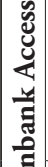 & రె & 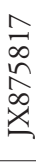 & $i$ & 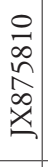 & 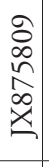 & I & 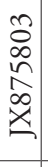 & 1 & i & 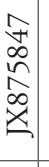 & 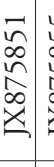 & 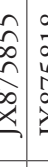 & 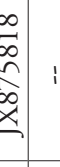 & $i$ & $i$ & $i$ & i & i & & 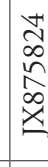 & 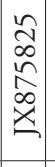 & 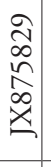 & 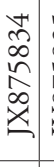 & $\begin{array}{l}n \\
\infty \\
\infty \\
\hat{n} \\
\infty \\
x\end{array}$ & 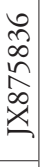 \\
\hline & $\mathfrak{N}$ & 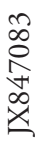 & 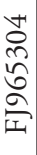 & 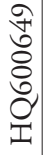 & i & 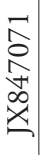 & 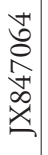 & 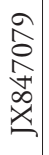 & 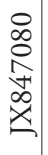 & 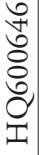 & 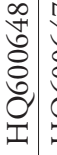 & 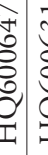 & 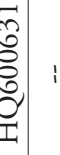 & $\mathrm{i}$ & i & \begin{tabular}{l}
$\vec{n}$ \\
0 \\
0 \\
$\hat{n}$ \\
\\
\cline { 2 - 2 }
\end{tabular} & 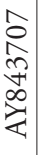 & i & 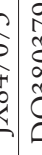 & $\mid \begin{array}{l}\vec{\sigma} \\
0 \\
0 \\
0 \\
0 \\
\text { I. }\end{array}$ & 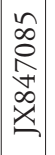 & 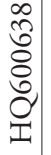 & 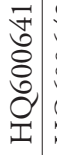 & 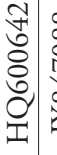 & 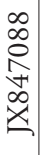 \\
\hline & 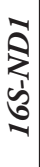 & 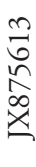 & i & 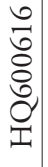 & $i$ & 胥 & $i$ & 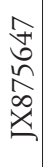 & 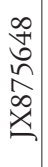 & 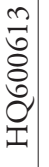 & 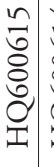 & 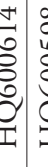 & 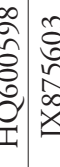 & 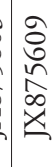 & $\left|\begin{array}{l}0 \\
\sigma \\
\curvearrowleft \\
\hat{\infty} \\
\underline{x}\end{array}\right|$ & i & i & 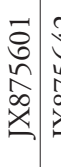 & & 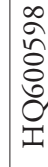 & 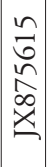 & 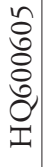 & $\begin{array}{l}0 \\
0 \\
0 \\
0 \\
0 \\
0 \\
0 \\
\end{array}$ & $\begin{array}{l}0 \\
0 \\
0 \\
0 \\
0 \\
0 \\
\end{array}$ & 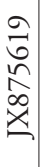 \\
\hline
\end{tabular}

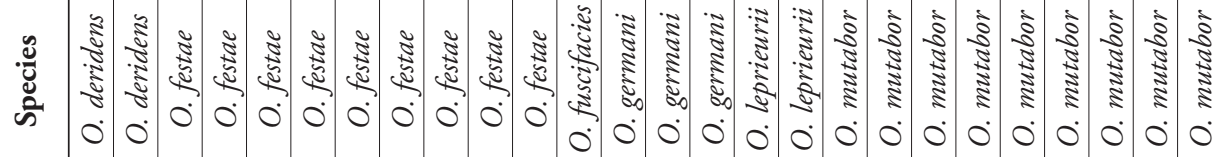

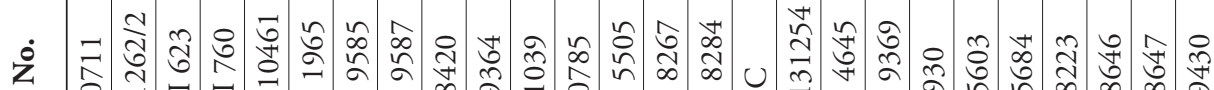

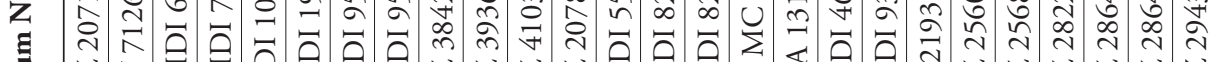

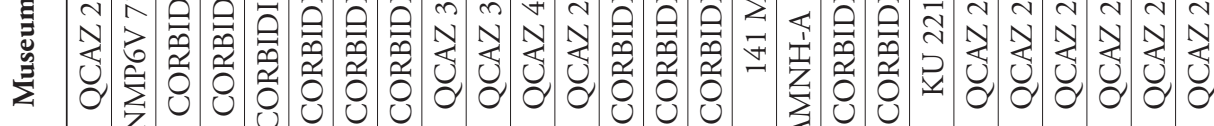




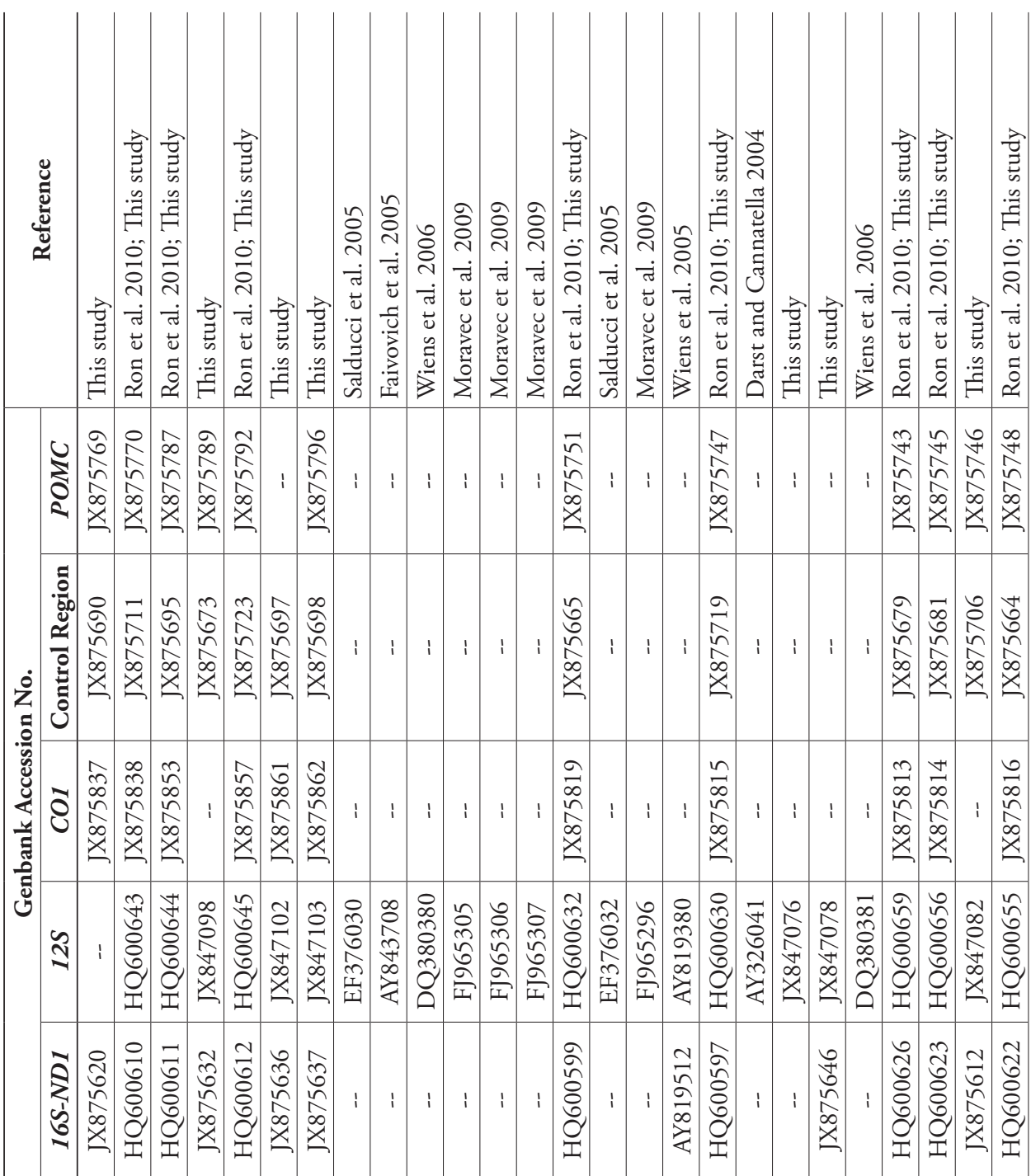

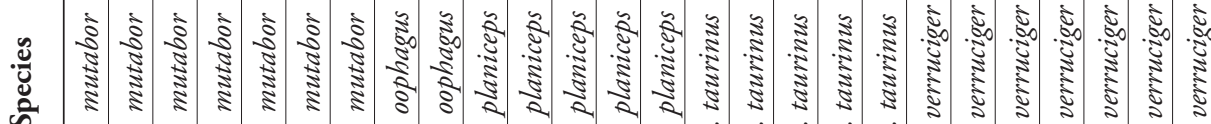

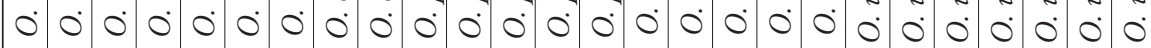

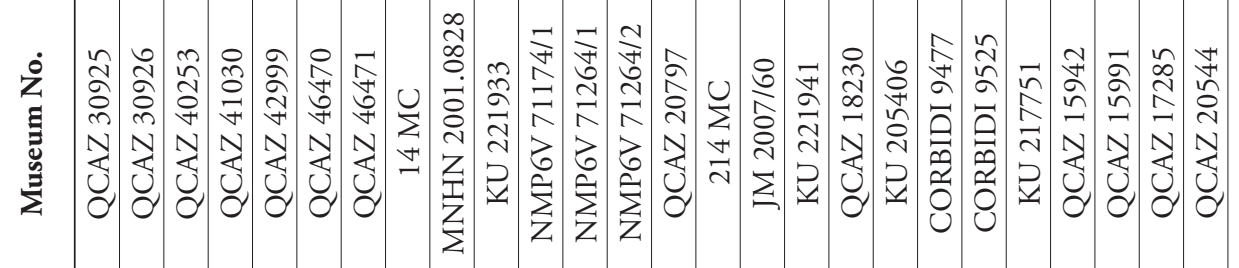




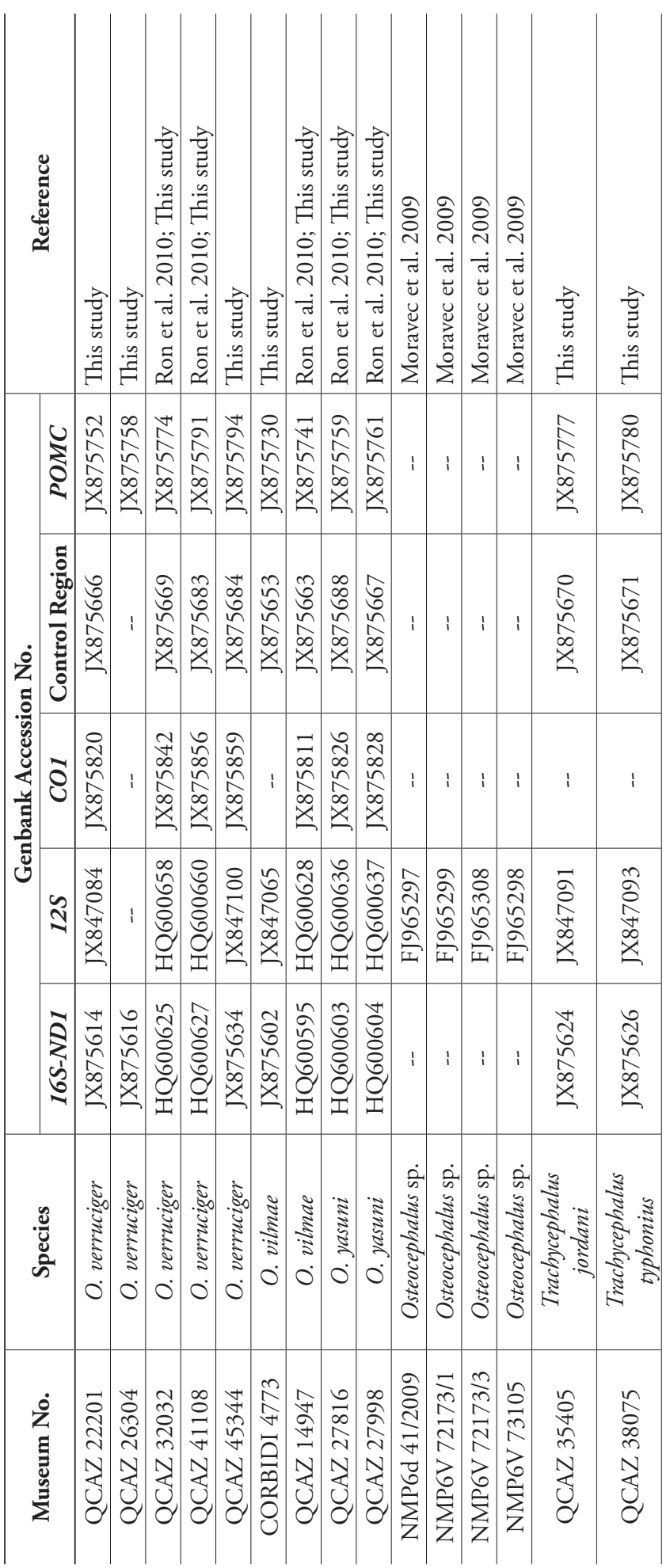




\section{Results}

\section{Phylogenetic analyses}

Throughout this section, genetic distances are uncorrected $p$-distances for gene $12 S$. The complete data set consists of up to six gene fragments (956 bp of $12 S, 325 \mathrm{bp}$ of $16 S, 693 \mathrm{pb}$ of CO1, $579 \mathrm{bp}$ of control region, $1079 \mathrm{bp}$ of $N D 1$, and $539 \mathrm{bp}$ of POMC) from 113 individuals representing 20 species. The models with the best fit and the estimated parameters for each partition for the Bayesian analyses are shown in Table 2. Comparisons of partition strategies based on Bayes factors favored the 12-partition analysis (factors values $>200$ ).

The topology (Fig. 1) is generally well supported and agrees with Salerno et al. (2012) phylogeny in showing a basal divergence between (O. taurinus + O. oophagus) and the other Osteocephalus species. Within the later, $O$. alboguttatus diverges basally while the remaining species are divided in two clades. One clade (posterior probability, PP, =1.0) corresponds to the $O$. buckleyi species group. The other clade has weaker support $(\mathrm{PP}=$ 0.91 ) and consists of the phytotelmata breeding species (O. planiceps, $O$. fuscifacies, $O$. deridens, $O$. castaneicola; $\mathrm{PP}=1.0$ ) and $O$. leprieurii, O. yasuni and $O . \mathrm{sp} . \mathrm{B}$ (sensu Moravec et al. 2009; $\mathrm{PP}=1.0)$. The "O. leprieurii" sample in the clade with phytotelmata breeding is likely misidentified as suggested by Moravec et al. (2009).

Table 2. Post burn-in averages for parameters of Bayesian analyses. Abbreviations are: $I=$ proportion of invariant sites, $G=$ shape parameter of the gamma distribution of rate variation.

\begin{tabular}{|c|c|c|c|c|c|c|c|c|c|c|c|c|c|}
\hline \multirow{2}{*}{ Partition } & \multirow{2}{*}{$\begin{array}{c}\text { Best-fit } \\
\text { model }\end{array}$} & \multirow{2}{*}{ I } & \multirow{2}{*}{ G } & \multicolumn{6}{|c|}{ Rate Matrix } & \multicolumn{4}{|c|}{ Base Frequency } \\
\hline & & & & AC & AG & AT & CG & CT & GT & A & $\mathrm{C}$ & G & $T$ \\
\hline $12 S$ & $\mathrm{GTR}+\mathrm{G}$ & - & 0.201 & 0.049 & 0.347 & 0.071 & 0.028 & 0.494 & 0.009 & 0.330 & 0.249 & 0.182 & 0.238 \\
\hline $16 S$ & $\mathrm{SYM}+\mathrm{I}+\mathrm{G}$ & 0.592 & 1.229 & 0.079 & 0.209 & 0.123 & 0.022 & 0.547 & 0.019 & - & - & - & - \\
\hline $\begin{array}{l}C O 1,1^{\text {st }} \\
\text { position }\end{array}$ & $\mathrm{K} 80+\mathrm{I}$ & & - & - & - & - & - & - & - & - & - & - & - \\
\hline $\begin{array}{l}C O 1,2^{\text {nd }} \\
\text { position }\end{array}$ & F81 & - & - & - & - & - & - & - & - & 0.168 & 0.269 & 0.155 & 0.408 \\
\hline $\begin{array}{l}C O 1,3^{\text {rd }} \\
\text { position }\end{array}$ & $\mathrm{GTR}+\mathrm{G}$ & - & 2.972 & 0.024 & 0.651 & 0.027 & 0.027 & 0.236 & 0.035 & 0.272 & 0.324 & 0.093 & 0.310 \\
\hline $\begin{array}{l}\text { Control } \\
\text { region }\end{array}$ & $\mathrm{HKY}+\mathrm{G}$ & - & 0.367 & - & - & - & - & - & - & 0.399 & 0.183 & 0.085 & 0.333 \\
\hline $\begin{array}{l}N D 1,1^{\text {st }} \\
\text { position }\end{array}$ & $\mathrm{HKY}+\mathrm{G}$ & - & 0.198 & - & - & - & - & - & - & 0.319 & 0.254 & 0.180 & 0.247 \\
\hline $\begin{array}{l}N D 1,2^{\text {nd }} \\
\text { position }\end{array}$ & $\mathrm{HKY}+\mathrm{I}+\mathrm{G}$ & 0.716 & 0.043 & - & - & - & - & - & - & 0.177 & 0.290 & 0.126 & 0.407 \\
\hline $\begin{array}{l}N D 1,3 \mathrm{rd} \\
\text { position }\end{array}$ & GTR+G & - & 1.780 & 0.032 & 0.601 & 0.036 & 0.018 & 0.295 & 0.019 & 0.353 & 0.273 & 0.098 & 0.275 \\
\hline $\begin{array}{l}\text { POMC, } 1^{\text {st }} \\
\text { position }\end{array}$ & $\mathrm{F} 81+\mathrm{G}$ & - & 0.128 & - & - & - & - & - & - & 0.412 & 0.169 & 0.259 & 0.160 \\
\hline $\begin{array}{l}\text { POMC, } 2^{\text {nd }} \\
\text { position }\end{array}$ & F81 & - & - & - & - & - & - & - & - & 0.419 & 0.183 & 0.199 & 0.199 \\
\hline $\begin{array}{l}\text { POMC, } 3^{\text {rd }} \\
\text { position }\end{array}$ & $\mathrm{HKY}+\mathrm{I}+\mathrm{G}$ & 0.485 & 0.761 & - & - & - & - & - & - & 0.318 & 0.319 & 0.164 & 0.199 \\
\hline
\end{tabular}




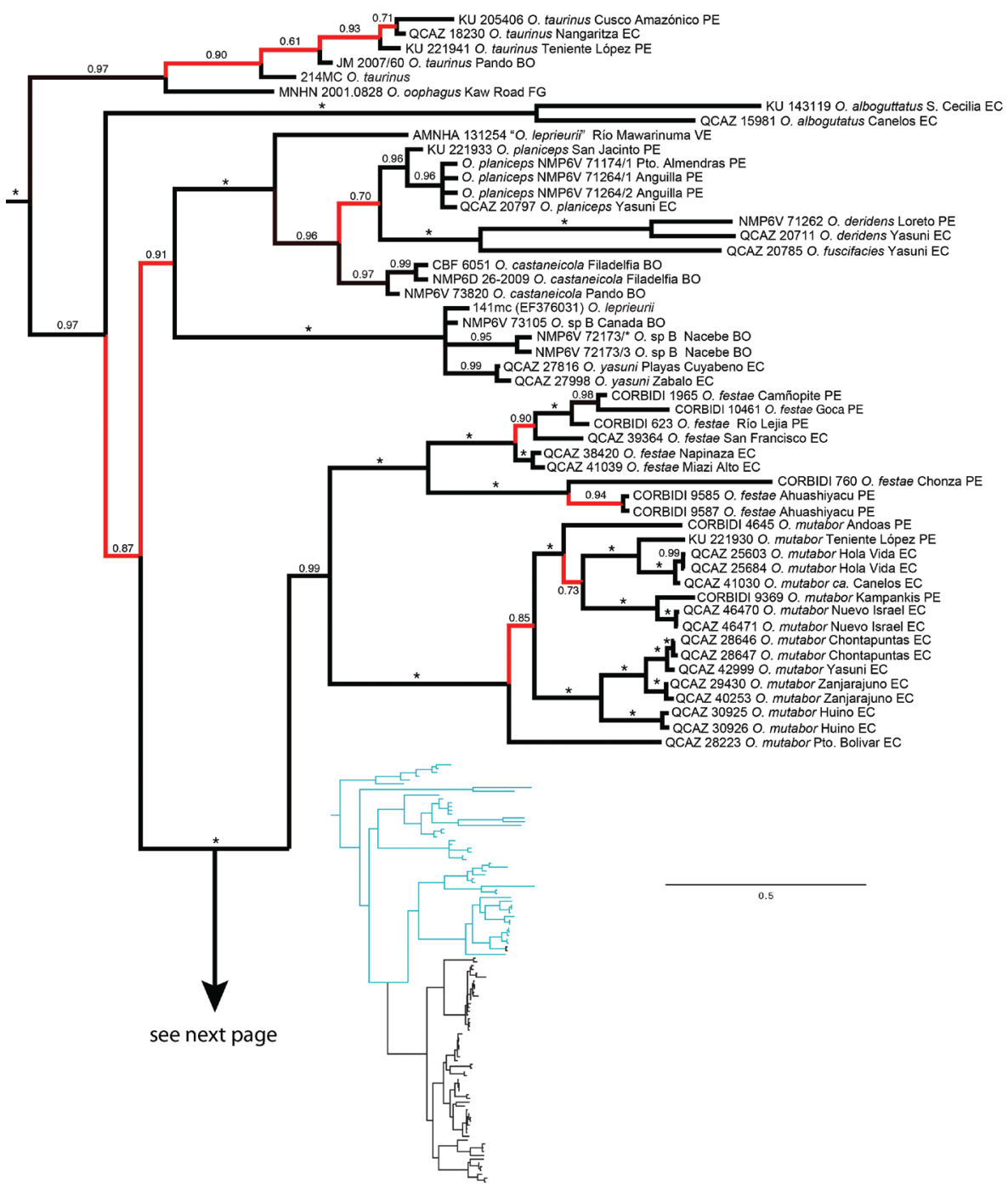

Figure I. Bayesian consensus phylogram depicting relationships within Osteocephalus. Phylogram derived from analysis of $4170 \mathrm{bp}$ of mitochondrial (gene fragments $12 \mathrm{~S}, 16 \mathrm{~S}, \mathrm{ND1}$, CO1, control region) and nuclear DNA (POM-C). Museum catalog no. (or, if unavailable, GenBank accession no.) and locality are shown for each sample. Posterior probabilities resulting from Bayesian Markov chain Monte Carlo searches appear above branches. An asterisk represents a value of 1 and red branches represent values $<0.95$. Outgroup species (Trachycephalus jordani and T. typhonius) are not shown. Abbreviations are: BO Bolivia, BR Brazil, CO Colombia, EC Ecuador, FG French Guiana, PE Peru, VE Venezuela.

All species within the $O$. buckleyi species complex, except $O$. buckleyi, are monophyletic. Individuals assignable to $O$. buckleyi are paraphyletic relative to $O$. verruciger and $O$. cabrerai and are separated in four clades (named A-D in Fig. 1).

Populations of $O$. mutabor segregate latitudinally: the most divergent population (Puerto Bolívar) is the only north of the Napo and Aguarico rivers; the remaining 


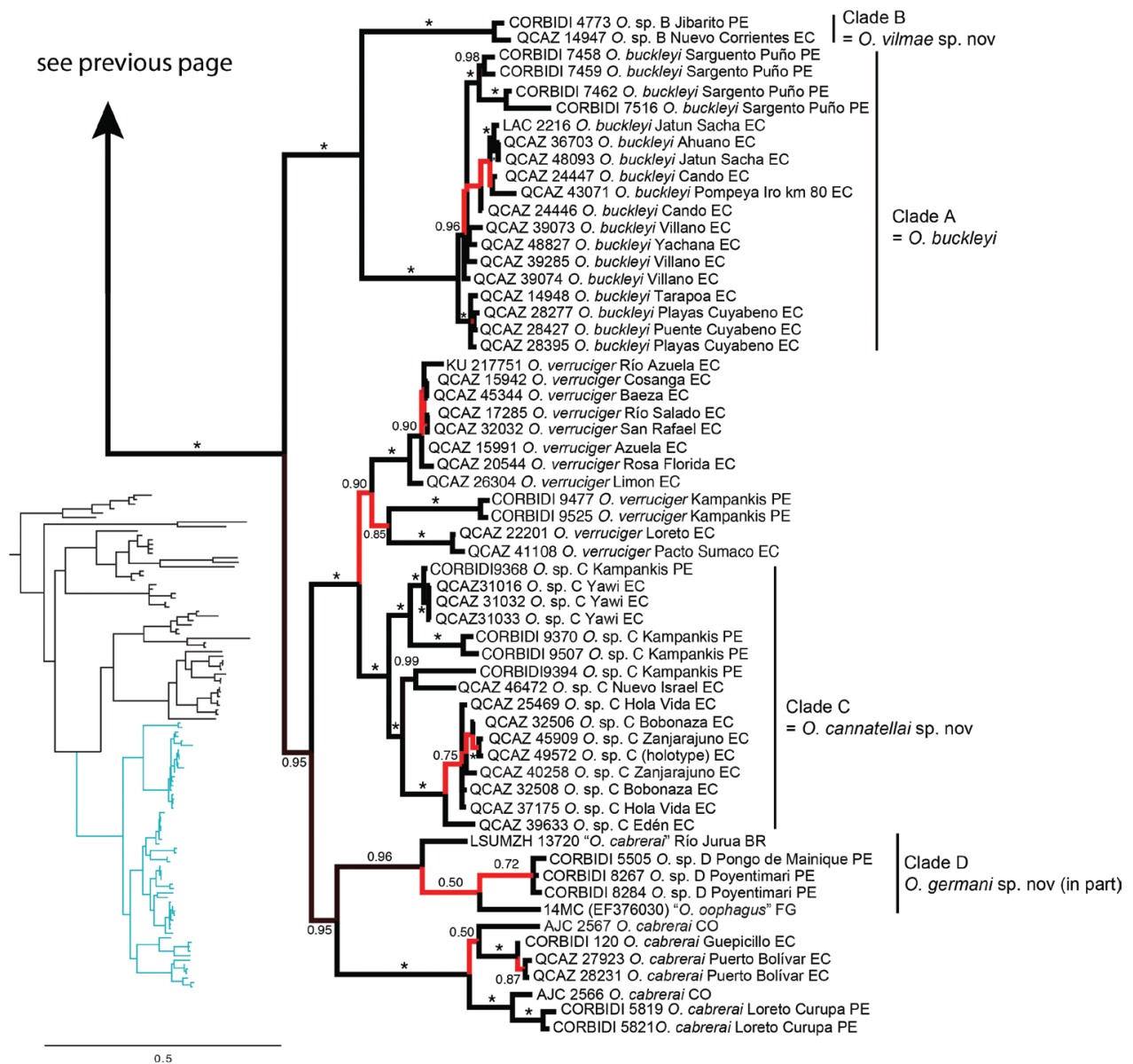

Figure I. Continued.

populations are separated in one central and one southern clade, both with strong support. Pairwise genetic distances between populations are below $2 \%$ in all comparisons.

The phylogeny recovers a monophyletic $O$. verruciger (in contrast to Ron et al. 2010) divided in two clades with an unexpected geographic pattern. Loreto and Pacto Sumaco are at a distance of 20-50 km from Cosanga, Río Salado and other nearby localities in central Ecuador (Fig. 2). Yet, in they phylogeny the two samples are sister to samples from Cordillera Kampankis in Peru, at a distance of $370 \mathrm{~km}$. Cordillera Kampankis is an isolated mountain range separated from the rest of the Andes by areas below $500 \mathrm{~m}$ above sea level. The records from Cordillera Kampankis are the first confirmed occurrences of O. verruciger in Peru. Genetic distances among O. verruciger samples range between 0 and 1.5\%.

Osteocephalus festae samples were collected on both sides of the dry valley of the Marañón River. This valley, with elevations as low as $600 \mathrm{~m}$, is part of the Huancabamba depression, a well-known biogeographic barrier in the Andes. Nevertheless, populations on both sides do not form reciprocally monophyletic groups. In some 


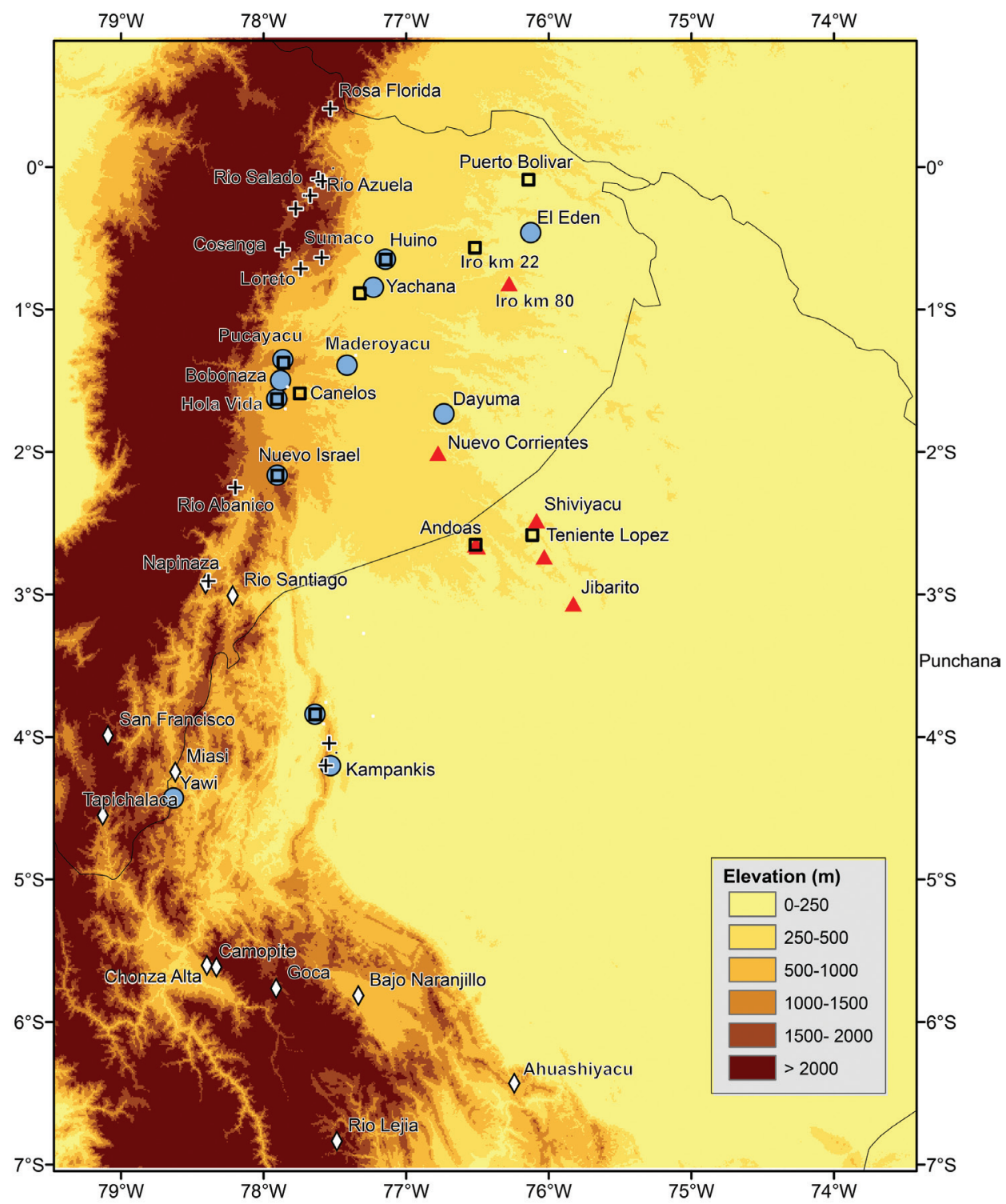

Figure 2. Records of Osteocephalus cannatellai, O. festae, O. mutabor, O. verruciger, and O. vilmae. Osteocephalus cannatellai, circles; $O$. festae, diamonds; $O$. mutabor, squares; $O$. verruciger, crosses; and $O$. vilmae, triangles. Locality data from the literature (Duellman and Mendelson 1995; Jungfer 2010; Peracca 1904; Ron et al. 2010) and specimens deposited at Museo de Zoología of Pontificia Universidad Católica del Ecuador, the Herpetology Collection, Escuela Politécnica Nacional, and Centro de Ornitología y Biodiversidad CORBIDI.

cases, low genetic distances (e.g., San Francisco-Camñopite $p$-distance 0.3\%) separate populations across the valley indicating recent gene flow. Relatively high genetic distances separate populations south of the Marańón (up to 2.8\% between Catarata Ahuashiyacu and Camñopite). 


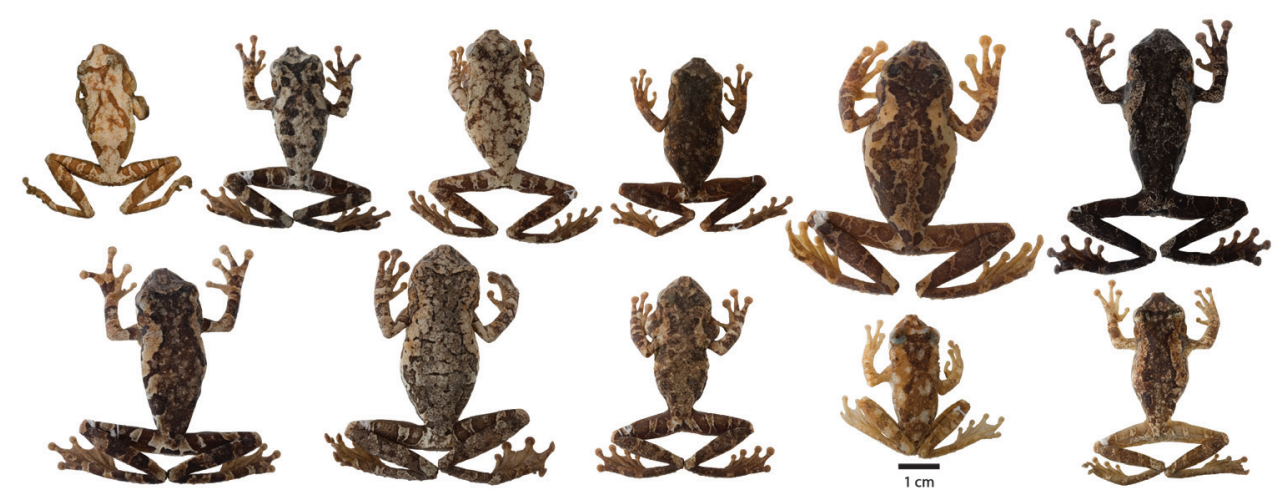

Figure 3. Variation in dorsal coloration of preserved specimens of adult Osteocephalus buckleyi. Left to right, upper row: BMNH 1947.2.13.44 (Lectotype), QCAZ 38704, EPN-H 6374, 11718 (males), QCAZ 2876, 14948 (females); lower row: QCAZ 39799, 26552 (females), 26488, 26561, 39364 (males). Provincia Napo, Orellana, Pastaza and Sucumbíos, Ecuador (See Appendix I for locality data). All specimens are shown at the same scale.

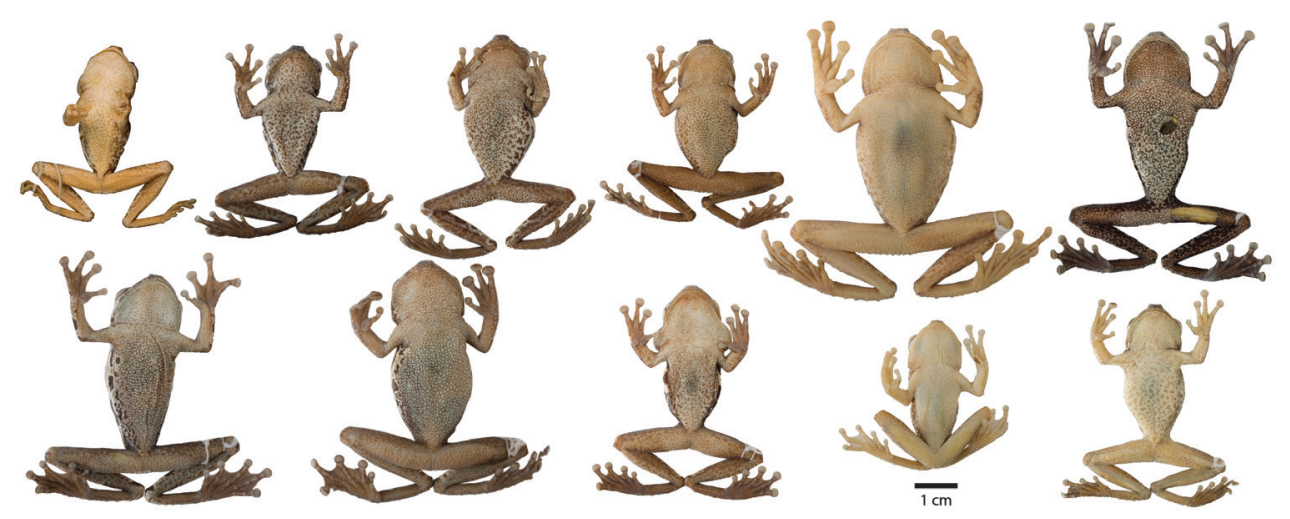

Figure 4. Variation in ventral coloration of preserved specimens of adult Osteocephalus buckleyi. Specimen identity and arrangement is the same as in Figure 3. All specimens are shown at the same scale.

Osteocephalus buckleyi-like individuals are grouped in four clades (A, B, C, and D in Fig. 1). Each clade has unique morphological features (see species descriptions) indicating that each represents a species. The external morphology of the lectotype of O. buckleyi (BMNH 1947.2.13.44, an adult male with nuptial excrescences, Figs 3-4) shows that it belongs to clade A because: (1) its body size $(37.90 \mathrm{~mm}$; Fig. 5) is within the range for adult males of Clade A (37.32-45.25 mm, $n=24)$ but below de range of clades B (48.23$51.85, n=4)$ and $\mathrm{C}(38.47-57.21 \mathrm{~mm}, n=24)$, (2) its relative tympanum size (tympanum diameter $/ \mathrm{SVL}=0.093$; Fig. 5) falls outside the range of Clade $\mathrm{C}(0.056-0.084$, $n=24$ males) but within the range of clade A (0.072-0.095, $n=24$ males), (3) it has conspicuous tarsal tubercles (absent in clade $\mathrm{D}$ ), and (4) clade $\mathrm{D}$ have a geographic range that, according to the available specimens, does not overlap with the type locality (Canelos, Provincia de Pastaza, Ecuador, $650 \mathrm{~m}$; Figs 2 and 6). Thus, we attach the binomial O. buckleyi to clade A. Clades B, C, and D cannot be assigned to any described species of 

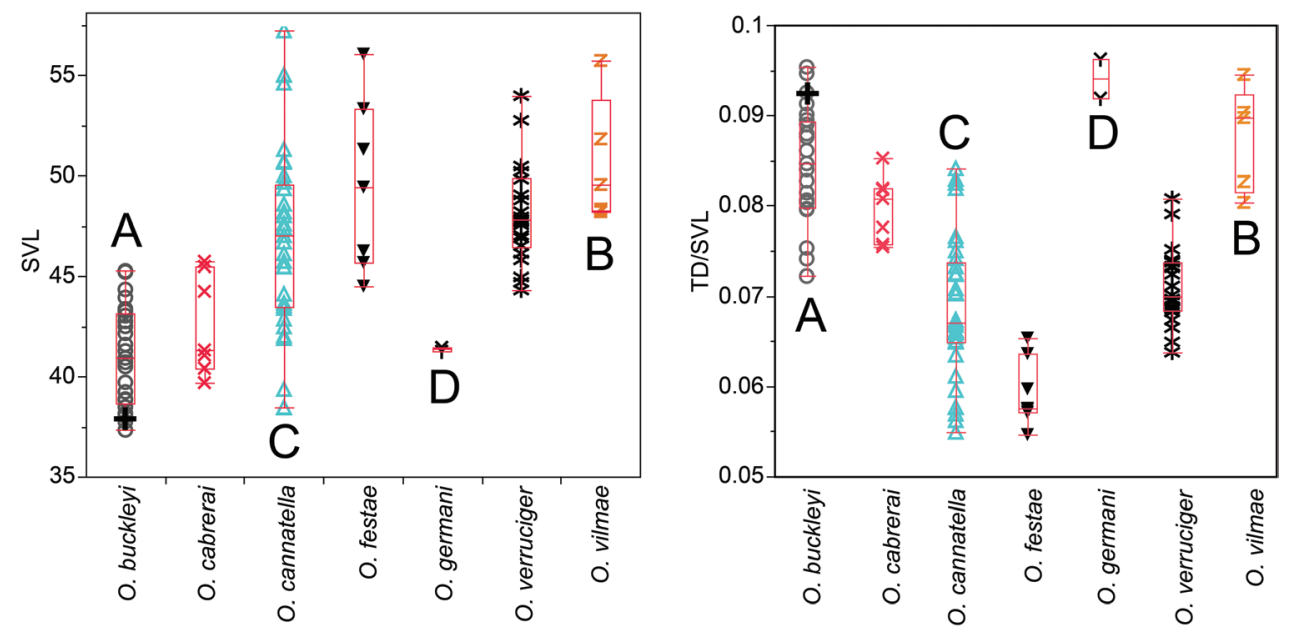

Figure 5. Boxplots for snout-vent length (SVL; left) and the ratio tympanum diameter/snout-vent length (TD/SVL; right). The line in the middle of the box represents the median, and the lower and upper ends of the box are the $25 \%$ and $75 \%$ quartiles respectively. Each individual is shown with a symbol; the cross in $O$. buckleyi represents the lectotype. Letters correspond to those of clades on Figure 1.

Osteocephalus and thus represent new species that we describe herein as $O$. cannatellai sp. n. (Clade C), O. germani sp. n. (part of Clade D), and O. vilmae sp. n. (Clade B).

Samples of $O$. buckleyi sensu stricto (clade A) have low genetic differentiation (uncorrected $p$ from 0 to $0.7 \%$ ) despite including localities separated by up to $450 \mathrm{~km}$. As in $O$. mutabor, the most divergent populations in the phylogeny were those north of the Napo and Aguarico rivers (Cuyabeno and Tarapoa).

Osteocephalus cannatellai sp. nov comprises eight populations with genetic distances ranging from 0 to $1.7 \%$. Populations group latitudinally forming a central and a southern clade. However, one of three samples (CORBIDI 9394) from the southern locality Pongo de Chinim (Kampankis) groups with the central localities.

Clade D comprises five samples from four populations. For two individuals (from Brazil and French Guyana) only GenBank sequences were available and thus we cannot determine if they belong to $O$. germani sp. $\mathrm{n}$. The three remaining samples (from Peru) are assigned to $O$. germani.

\section{Species accounts}

\section{Osteocephalus cannatellai sp. n.}

urn:lsid:zoobank.org:act:EDEC6BF4-F11C-4812-A06E-065C9035999D

http://species-id.net/wiki/Osteocephalus_cannatellai

Holotype. (Figs 7-10) QCAZ 49572 (field no. PUCE 18835), adult male from Ecuador, Provincia Pastaza, Cantón Santa Clara, Río Pucayacu, in the vicinities of the 
Zanjarajuno Reserve $\left(1.3578^{\circ} \mathrm{S}, 77.8477^{\circ} \mathrm{W}\right), 940 \mathrm{~m}$ above sea level, collected by $\mathrm{P}$. Peña-Loyola, N. Peñafiel, and R. Tarvin on 3 July 2010.

Paratopotypes. 20 adult males, 1 adult female. QCAZ 33256, adult male, collected by I. G. Tapia, D. Almeida-Reinoso and M. Páez on 30 March 2007; QCAZ 39579, 39586-87, adult males, collected by D. Salazar-Valenzuela and G. Diaz between 12 and 14 December 2008; QCAZ 40909-10, adult males, collected by I. G. Tapia, L. A. Coloma, and S. R. Ron on 31 March 2008; QCAZ 40252, 40258, adult males, collected by D. Salazar-Valenzuela, D. Acosta-López and C. Korfel between 23 February and 1 March 2009; QCAZ 45271-72, 45277, 45281, adult males, collected by D. AcostaLópez between 30 July and 2 August 2009; QCAZ 45907, 45909, adult males, collected by P. Peña-Loyola on 16 October 2009; QCAZ 49569-71, adult males, collected by N. Peñafiel between 26 June and 3 July 2010; QCAZ 49021-22, adult males, collected by R. Tarvin, and L. Bustamante on 3 August 2010; QCAZ 49439, adult female, collected by R. Tarvin and P. Aguilar on September 2010; QCAZ 48744 adult male, collected by S. R. Ron, L. Bustamante, I. G. Tapia, P. Peña-Loyola and R. Tarvin on 3 July 2010.

Paratypes. 42 adult males, 2 adult females. Ecuador: Provincia Morona Santiago: Bobonaza $\left(1.4980^{\circ} \mathrm{S}, 77.8793^{\circ} \mathrm{W}\right), 660 \mathrm{~m}$ above sea level, QCAZ 32506, 32508, 32512, adult males, collected by L. A. Coloma and I. G. Tapia on 18 August 2008; Nuevo Israel $\left(2.165^{\circ} \mathrm{S}\right.$, $\left.77.902919^{\circ} \mathrm{W}\right), 1289 \mathrm{~m}$ above sea level, QCAZ 46472, adult male, collected by J. BritoMolina on 2 January 2010; Provincia Napo: Reserva Yachana $\left(0.8458^{\circ} \mathrm{S}, 77.2287^{\circ} \mathrm{W}\right)$, 300-350 m above sea level, QCAZ 48790, 48797, 48803-04, 48811, 48814, adult males, collected by S. North, S. Topp and G. Estevez between 4 June and 18 August 2008; Huino, QCAZ 50198, adult female, collected by W. C. Funk on February 2003; Provincia Orellana: El Edén $\left(0.46147^{\circ} \mathrm{S}, 76.1252^{\circ} \mathrm{W}\right), 228 \mathrm{~m}$ above sea level, QCAZ 39633, adult male, collected by S. Aldás-Alarcón, Dayuma, Pozo Sunka $\left(1.7333^{\circ} \mathrm{S}, 76.7333^{\circ} \mathrm{W}\right), 279 \mathrm{~m}$ above sea level, EPN-H 2752, 2755-56, 6372; Provincia Pastaza: Fundación Hola Vida $\left(1.6285^{\circ} \mathrm{S}, 77.9072^{\circ} \mathrm{W}\right), 845 \mathrm{~m}$ above sea level, QCAZ 25607, 25469, adult males, collected by K. Elmer and I. G. Tapia on 27 June 2003, QCAZ 37175, adult male collected by I. G. Tapia, L. A. Coloma, P. Peña-Loyola and M. Páez on July 2007; Río Maderoyacu $\left(1.3917^{\circ} \mathrm{S}, 77.4139^{\circ} \mathrm{W}\right), 500-600 \mathrm{~m}$ above sea level, EPN-H 6373, 6385, adult males, collected by A. Almendáriz; Provincia Zamora Chinchipe: Centro Shuar Yawi $\left(4.4300^{\circ} \mathrm{S}\right.$, $\left.78.6316^{\circ} \mathrm{W}\right), 945 \mathrm{~m}$ above sea level, QCAZ 31016, 31032-33, 31047, 31053, adult males, QCAZ 31051, adult female, collected by D. Almeida-Reinoso and A. Armijos between 13 and 19 September 2003. Peru: Región Loreto: Provincia Datem del Marańón: Cordillera de Kampankis: Pongo de Chinim $\left(3.1130^{\circ} \mathrm{S}, 77.7762^{\circ} \mathrm{W}\right), 365 \mathrm{~m}$ above sea level, CORBIDI 09368, 09370, 09394, 9396, 10534, 10537, MUSM 28050, adult males collected by P. J. Venegas and A. Catenazzi on 3 August 2011; Quebrada Kampankis $\left(4.0431^{\circ} \mathrm{S}, 77.5412^{\circ} \mathrm{W}\right.$ ), $325 \mathrm{~m}$ above sea level, CORBIDI 09507, 10535, adult males collected by P. J. Venegas and A. Catenazzi on 13 August 2011; Quebrada Wee (4.2041 $\left.{ }^{\circ} \mathrm{S}, 77.5298^{\circ} \mathrm{W}\right), 310 \mathrm{~m}$ above sea level, CORBIDI 09545-46, 09553, 09569, 10532-33, 10535-36, MUSM 28051, adult males, collected by P. J. Venegas and A. Catenazzi on 18 August 2011.

Diagnosis. Throughout this section, coloration refers to preserved specimens unless otherwise noted. Osteocephalus cannatellai is a medium-sized species of Osteocepha- 


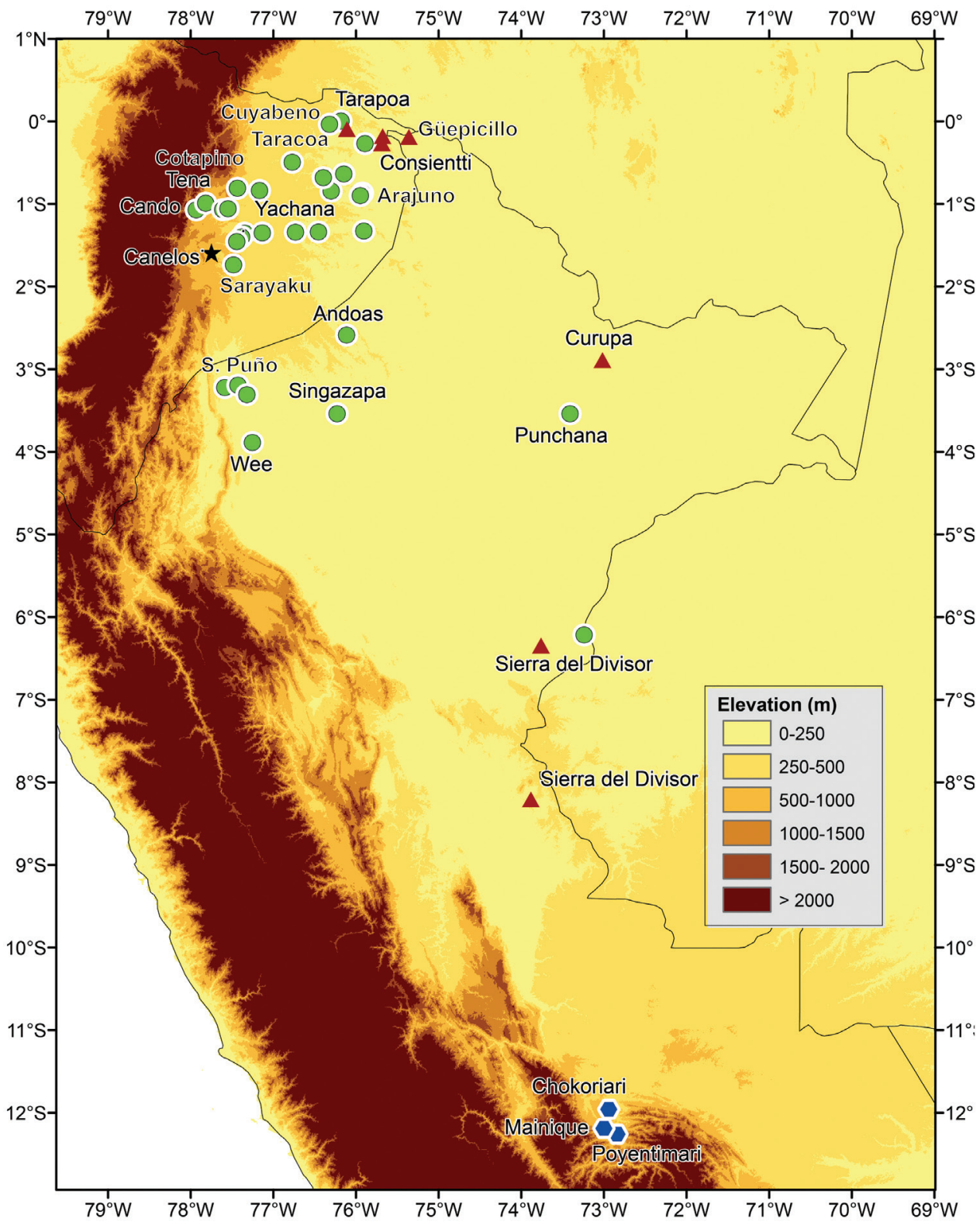

Figure 6. Records of Osteocephalus buckleyi, O. cabrerai, and O. germani. Osteocephalus buckleyi, circles; $O$. cabrerai, triangles, and $O$. germani, hexagons. The type locality of $O$. buckleyi is shown with a star. Locality data from the literature (Duellman and Mendelson 1995; Jungfer 2010; Peracca 1904; Ron et al. 2010) and specimens deposited at Museo de Zoología of Pontificia Universidad Católica del Ecuador, the Herpetology Collection, Escuela Politécnica Nacional, and Centro de Ornitología y Biodiversidad CORBIDI.

lus having the following combination of characters: (1) size sexually dimorphic; maximum SVL in males $57.21 \mathrm{~mm}(n=33)$, in females $70.88(n=3)$; (2) skin on dorsum bearing scattered tubercles in males, smooth in females; (3) skin on flanks areolate; (4) 

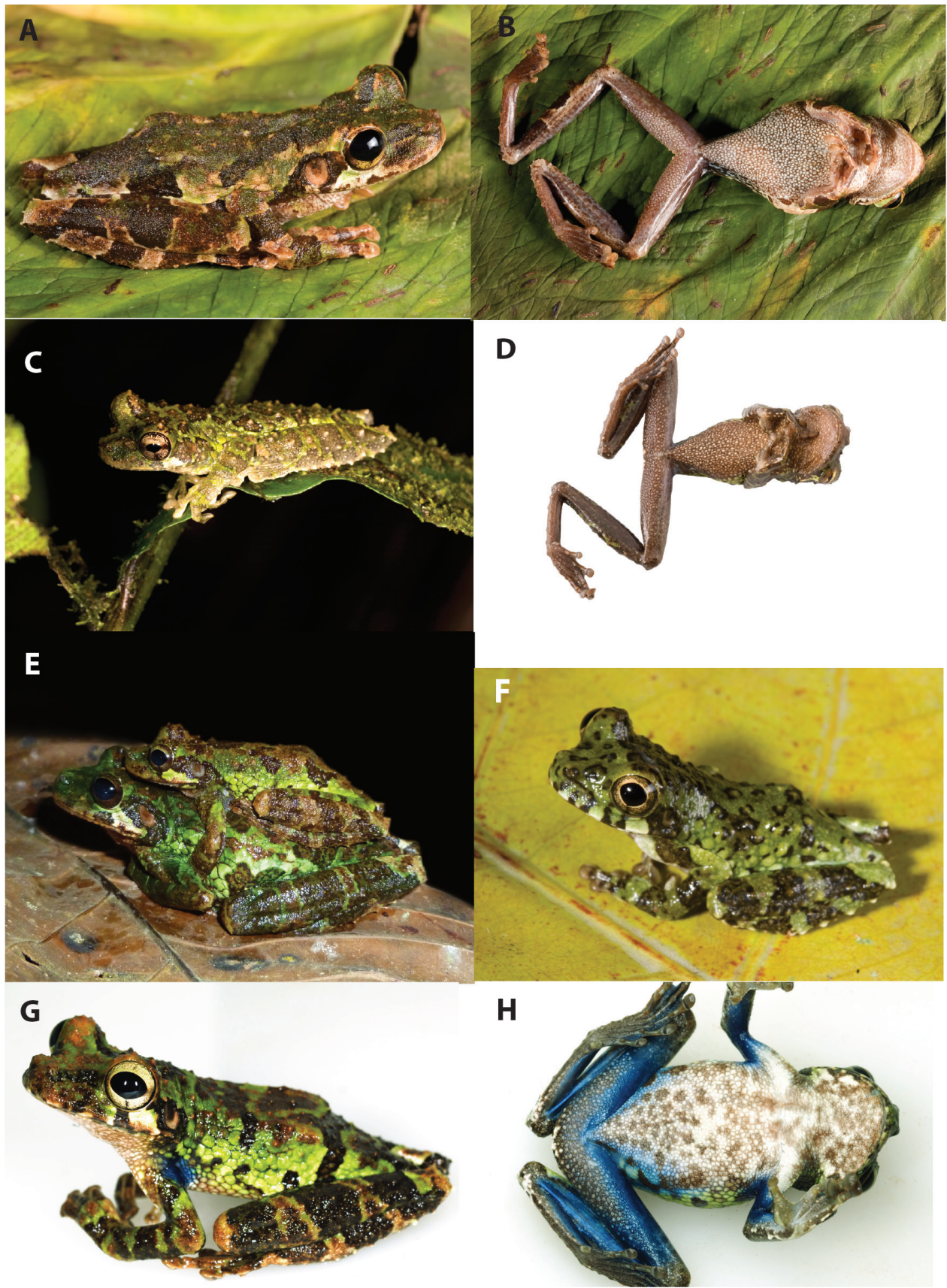

Figure 7. Dorsolateral and ventral views of Osteocephalus. A-B Osteocephalus buckleyi, QCAZ 43071, adult female, SVL $=50.95 \mathrm{~mm}$, Jatun Sacha, Ecuador C-D O. cannatellai QCAZ 48744, adult male, SVL $=51.96 \mathrm{~mm}$, Reserva Zanjarajuno, Río Pucayacu, Ecuador E O. cannatellai, amplectant pair QCAZ 49572 (holotype) adult male, SVL $=52.85 \mathrm{~mm}$, from Río Pucayacu, Provincia Pastaza, Ecuador F $O$. cannatellai QCAZ 40859, juvenile, SVL $=26.7 \mathrm{~mm}$ (type locality) G-H Osteocephalus cannatellai (CORBIDI 10534) from Cordillera Kampankis, Peru. Photographs E by R. Tarvin and G-H by A. Catenazzi. 
hand webbing formula varying from I basal II $1 \frac{1 / 2}{2}-2^{1 /} / \mathrm{III}^{+}-2 \mathrm{IV}$ to I basal II $2^{-}-3^{-}$ $\mathrm{III} 2^{2} /{ }_{3}-2^{1 / 2 \mathrm{IV}}$; foot webbing formula varying from I1 $-2 \frac{1}{2} \mathrm{II} 1-2 \mathrm{III} 1^{+}-2 \mathrm{IV} 2^{1 / 2}-$ $1^{+} \mathrm{V}$ to $\mathrm{I}^{+}-1^{-} \mathrm{II} 0^{+}-1^{+} \mathrm{III} 0^{+}-1 \frac{1}{2} / 2 \mathrm{IV}^{-}-0^{+} \mathrm{V}$; (5) dorsum varying from dark brown with light gray marks to cream with brown marks; (6) venter varying from light gray to dark brown with lighter dots and/or dark brown blotches; (7) cream suborbital mark present, clear labial stripe absent; (8) flanks cream with darker reticulations anteriorly and dark marks; (9) dermal roofing bones of the skull weakly exostosed; (10) in life, bones green; (11) in life, iris bronze with irregular reticulations; (12) paired vocal sacs small, located laterally, behind jaw articulation, (13) in life, juveniles with bronze iris, without pale elbows, knees, and heels; (14) larvae unknown.

Osteocephalus cannatellai is most similar to O. buckleyi and O. vilmae sp. n. The three species differ from other Osteocephalus by the combination of a bronze iris with irregular black reticulations (in life), areolate skin on the flanks, prominent tubercles in the tarsus and absence of a row of conspicuous tubercles in the lower jaw. Osteocephalus cannatellai differs from O. buckleyi in having: (1) scattered and weakly keratinized dorsal tubercles (more abundant and keratinized in $O$. buckleyi), (2) smaller tympanum (1/5 of head length in $O$. cannatellai vs. $1 / 4$ in $O$. buckleyi; Fig. 5), (3) larger size (O. cannatellai mean male $\mathrm{SVL}=46.83, \mathrm{SD}=4.31, n=33$; O. buckleyi mean male SVL $=41.12, \mathrm{SD}=2.45, n=24$; differences are significant: $t=5.82, P<0.001$; Fig. 5), (4) darker venter (cream with brown speckling in most O. buckleyi; Figs 4 and 9.), (5) more extensive areolate area on flanks (from axillary region to groin in O. cannatellai, restricted to anterior one third of flank in O. buckleyi), (6) contrasting coloration between flanks and venter (change in coloration is gradual in O. buckleyi), and (7) advertisement call (Fig. 11). Our phylogenetic analyses show that $O$. cannatellai and O. buckleyi are not sister species (Fig. 1).

Osteocephalus cannatellai differs from $O$. vilmae in having a narrower head (relative to SVL, mean male $\mathrm{HW} / \mathrm{SVL}=0.323, \mathrm{SD}=0.034, n=33$; $O$. vilmae mean male $\mathrm{HW} / \mathrm{SVL}=0.355, \mathrm{SD}=0.012, n=5$; differences are significant: $t=2.06, P=0.046$ ) and a smaller tympanum (relative to SVL, mean male TD/SVL $=0.069, \mathrm{SD}=0.007$, $n=33$; $O$. vilmae mean male $\mathrm{TD} / \mathrm{SVL}=0.087, \mathrm{SD}=0.006, n=5$; differences are significant: $t=5.17, P<0.001)$. According to the phylogeny, $O$. cannatellai and $O$. vilmae are not sister species (Fig. 1). Osteocephalus cannatellai differs from $O$. cabrerai in (1) lacking prominent tubercles on the lower jaw, (2) having smooth to tuberculate outer edge of Finger IV (outer edge with fringe in O. cabrerai), and (3) having less webbing in the hands (in $O$. cannatellai webbing reaches two thirds of the distance between the ultimate and penultimate tubercle of Finger IV, in O. cabrerai it reaches the proximal border of the ultimate tubercle; Fig. 12). Osteocephalus cannatellai differs from other species of Osteocephalus (except O. buckleyi, O. cabrerai, and O. vilmae) in having a combination of prominent tubercles in the tarsus and areolate skin in the flanks. A bronze iris with black reticulations further distinguishes $O$. cannatellai from $O$. deridens, $O$. oophagus, $O$. planiceps, and $O$. taurinus which have black straight lines radiating from the pupil; iris coloration also differs in $O$. carri, O. festae, O. heyeri, O. subtilis, and $O$. verruciger which have predominantly dark irises (Jungfer 2010; Jungfer 
and Lehr 2001; Lynch 2002). Osteocephalus cannatellai is larger than O. exophthalmus (maximum male SVL in O. cannatellai 57.21, $n=33$; in O. exophthalmus $32.7 \mathrm{~mm}, n$ = 3; Smith and Noonan 2001) and O. fuscifacies (maximum SVL 44.17, $n=21$ ). Skin texture in the flanks distinguishes O. cannatellai (areolate) from O. mutabor (smooth). Osteocephalus inframaculatus differs from O. cannatellai in coloration of the ventral surfaces of hindlimbs (bold brown blotches in O. inframaculatus are absent in O. cannatellai; Jungfer 2010).

Description of holotype. Adult male, $52.85 \mathrm{~mm} \mathrm{SVL}$, head length 18.61, head width 18.53 , eye diameter 5.08, tympanum diameter 3.31, femur length 25.84 , tibia length 30.05, foot length 22.73. Head narrower than body, slightly longer than wide; snout truncate in lateral and dorsal views; distance from nostril to eye longer than diameter of eye; canthus rostralis distinct and rounded; loreal region concave; internarial area depressed; nostrils moderately protuberant, directed laterally; interorbital area flat, lateral margins of the frontoparietals inconspicuous through skin; eye large, strongly protuberant; tympanic membrane clearly evident, large, slightly wider than high, about two thirds of eye diameter, separated from eye by ca. $85 \%$ of its diameter; tympanic annulus distinct except dorsally where it is covered by supratympanic fold; posterior end of supratympanic fold reaches arm insertion. Arm slender, axillary membrane present, reaching one third of arm length; four small low tubercles present along ventrolateral edge of forearm; relative length of fingers $\mathrm{I}<\mathrm{II}<\mathrm{IV}<\mathrm{III}$; fingers bearing large, oval discs, that of third finger about three fourths of tympanum diameter; subarticular tubercles prominent, round to ovoid, single; supernumerary tubercles present; palmar tubercle small, elongated; prepollical tubercle large, flat, elliptical; prepollex enlarged; large dark keratinous nuptial excrescences covering inner surface of prepollex up to half the distance between subarticular tubercle and proximal border of disk of thumb; webbing formula of fingers I basal II $12 / 3-2{ }_{3}^{2}$ III $2 \frac{1}{2}-2+\mathrm{IV}$. Medium sized to small tubercles on tibiotarsal articulation; scattered tubercles on tarsus, more abundant on outer edge; small tubercles scattered along ventrolateral edge of foot; toes bearing discs slightly wider than long, smaller than those of fingers; relative length of toes $\mathrm{I}<\mathrm{II}<\mathrm{V}<\mathrm{III}<\mathrm{IV}$; outer metatarsal tubercle ill defined, small, round; inner metatarsal tubercle large, ovoid; subarticular tubercles single, round, protuberant; supernumerary tubercles restricted to the soles; webbing formula of toes I1 $-2 \mathrm{II} 1-2^{+} \mathrm{III} 1^{+}-2+\mathrm{IV} 2^{-}-1 \mathrm{~V}$. Skin on dorsum, head, and dorsal surfaces of limbs smooth, with scattered tubercles; skin on flanks areolate; skin on venter coarsely granular; skin on ventral surfaces of head and thighs granular, those of shanks smooth. Cloacal opening directed posteriorly at upper level of thighs; short simple cloacal sheath covering cloacal opening; round tubercles below vent; two conspicuous white tubercles ventrolateral to vent. Tongue cordiform, widely attached to floor of mouth; dentigerous processes of the vomer angular, adjacent medially, posteromedial to choanae, bearing 12 and 9 (left/right) vomerine teeth; choanae trapezoidal, oblique; vocal sac barely distinct above the arm and below the ear.

Color of holotype in preservative. Dorsum brown with light gray to cream peripheral marks; dark brown, ill defined, transversal bar between orbits (Fig. 8); cream middorsal line from tip of snout to end of sacrum; dorsal surfaces of forearms brown with light 


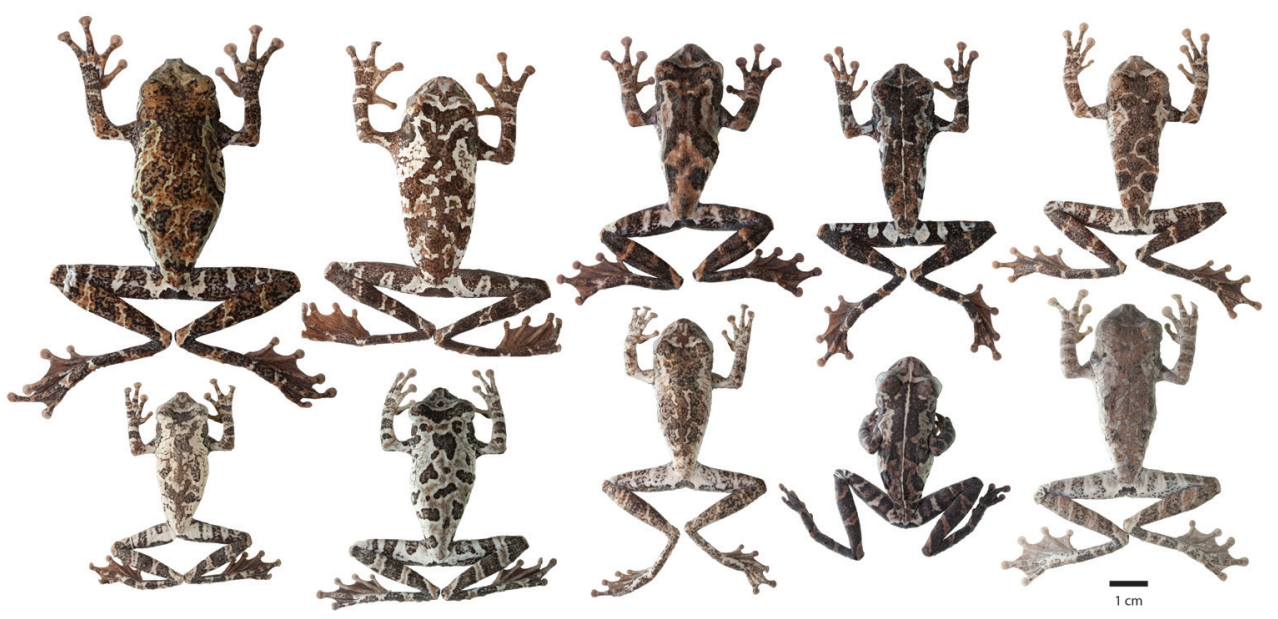

Figure 8. Adult Osteocephalus cannatellai showing variation in dorsal coloration of preserved specimens. Left to right, upper row (dark morphs): QCAZ 49439, 31051 (females), 40258, 49022, 45271 (males); lower row (light morphs): QCAZ 37175, 487444, 48797, 39633, 46472 (males). Ecuador, Provincia Morona Santiago, Napo, Orellana, Pastaza and Zamora Chinchipe. All specimens are shown at the same scale.

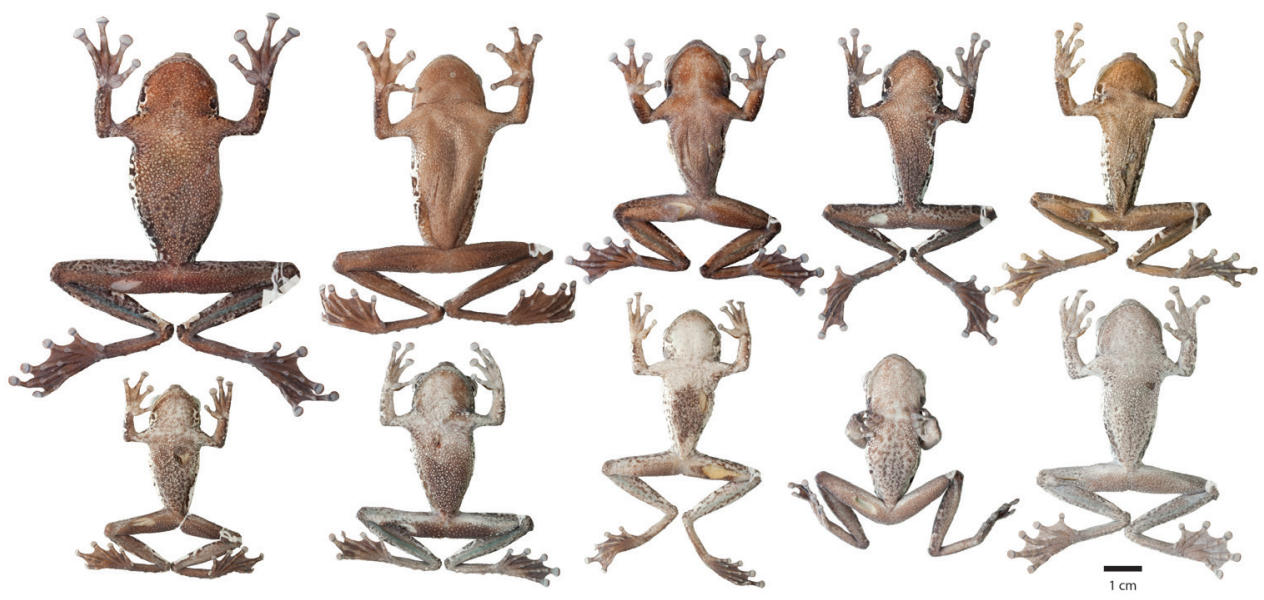

Figure 9. Adult Osteocephalus cannatellai showing variation in ventral coloration of preserved specimens. Specimen identity and arrangement is the same as in Figure 8. All specimens are shown at the same scale.

gray and dark gray marks, dorsal surfaces of thighs light gray with dark gray transversal bands, dorsal surfaces of shanks and feet brown with dark gray marks. Venter brown with light cream yellowish spots, more abundant on posterior half of the body (Fig. 9); ventral surfaces of hindlimbs and forelimbs brown with dark brown marks and conspicuous white tubercles on forearms; outer half of ventral surfaces of forearms dark brown; sides of head brown with oblique white bar from posteroventral border of orbit to border of jaw, below tympanum (Fig. 10); vertical dark brown bar below eye, anterior to white bar; area behind white bar and eye dark brown except for brown 


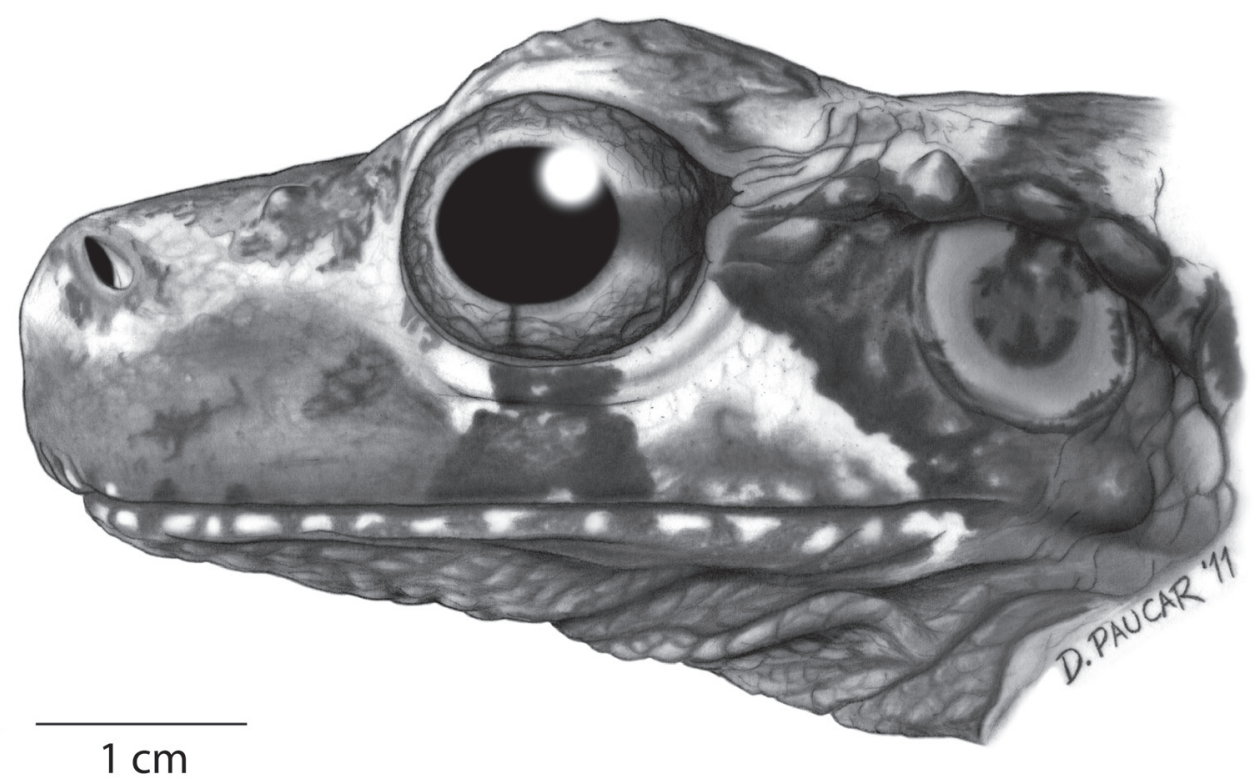

Figure 10. Lateral view of the head of the holotype of Osteocephalus cannatellai (QCAZ 49572).

periphery of tympanum; iris light gray with black reticulations; flanks light gray anteriorly, cream posteriorly, areolate region with gray reticulation.

Color of holotype in life. Based on digital photographs. Dorsum brown with green peripheral marks; dark brown, ill defined, transversal bar between orbits (Fig. 7E); dorsal surfaces of forearms brown with green and dark brown marks, dorsal surfaces of thighs dark green with dark brown transversal bands, dorsal surfaces of shanks and feet brown with dark brown marks. Sides of head brown with oblique lime bar from posteroventral border of orbit to border of jaw, below tympanum; vertical dark brown bar below eye, anterior to lime bar; area posterior to lime bar and eye dark brown except for brown periphery of tympanum; flanks light green, areolate region with dark reticulation.

Etymology. The specific name cannatellai is a noun in the genitive case and is a patronym for David C. Cannatella, who with his research has enriched the understanding of the evolution of Neotropical amphibians. He has also contributed to amphibian studies in Ecuador by providing funding and training to local scientists.

Variation. Variation in dorsal and ventral coloration of preserved specimens is shown in Figures 8 and 9. Dorsal background coloration varies from cream to light gray or brown; irregular dark brown or dark gray marks are always present (Fig. 8). Some specimens have a cream middorsal line from the tip of the snout to the mid sacrum (QCAZ 49570) or the vent (QCAZ 39633). In females, the dorsum always lacks tubercles while in males it varies between lacking tubercles (QCAZ 32508) and having scant and ill-defined non-keratinized tubercles (e.g., QCAZ 48814 and 49569). The prominence of the tubercles seems to decrease in preserved specimens: when collected, QCAZ 48744 had large conspicuous dorsal tubercles (Fig. 7), in preservative tubercles are barely noticeable. 


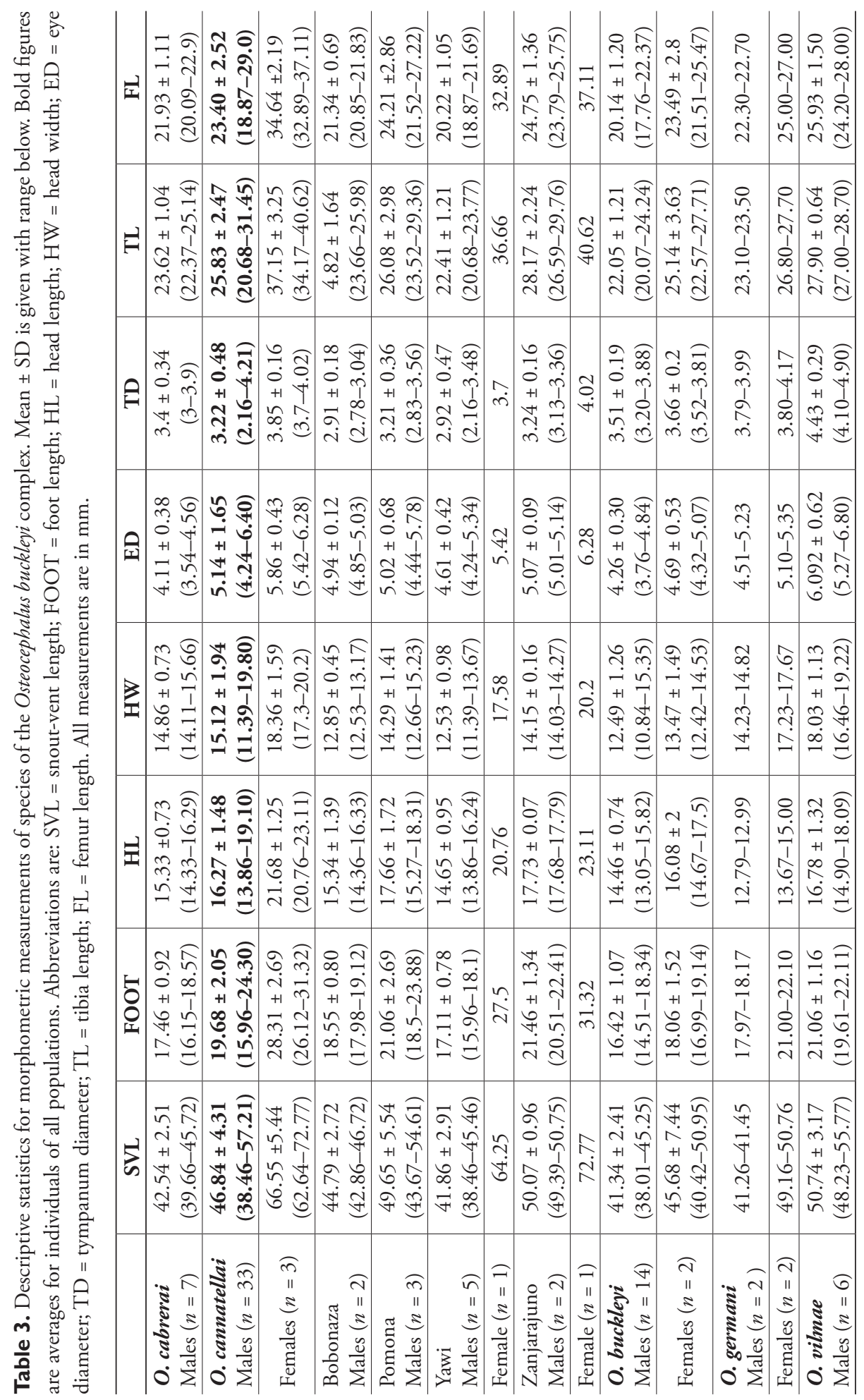


Ventral surfaces of preserved specimens (Fig. 9) vary from light gray (QCAZ 40909) to brown (QCAZ 31031). In most specimens, there are dark brown or dark gray spots, more abundant posteriorly (e.g., QCAZ 49439); QCAZ 39633 has brown blotches on the chest and venter; QCAZ 48804 has similar marks that also reach the gular region. In two Peruvian specimens ventral surfaces are light gray with few brown spots posteriorly (CORBIDI 09553) or with light brown spots, slightly visible, on gular region and belly (MUSM 28050). The gular regions in some Peruvian specimens are brown (e.g., CORBIDI 09507, 10532). Ventrally, limbs vary from light gray or light brown to dark brown; in QCAZ 33256 and 39587 black dots are present on limbs; scant cream tubercles can be present in the external edge of the forearm (e.g., QCAZ 32512). The skin of the anterior and posterior surfaces of thighs and the concealed surfaces of shanks are pale in the Peruvian specimens. The vent region is light gray to dark brown with dark brown dots. Flanks are cream to light gray, areolate in the anterior two-thirds and smooth posteriorly. In specimens from Peru the flanks are completely areolate. The areolate portion has a dark brown reticulation.

Head shape is truncate in dorsal and lateral view (e.g., QCAZ 39579). Lateral head coloration varies between dark brown (QCAZ 49569) to cream (QCAZ 32506). There is a cream subocular mark. The tympanic annulus is concealed dorsally and has lighter color than the background. The distal subarticular tubercle on Finger IV is single (e.g., QCAZ 40909) or bifid (e.g., QCAZ 45272).

Morphometric data pertain to adults and are summarized in Table 3. In the examined series, the largest male has a SVL of $57.21 \mathrm{~mm}$ and the largest female 72.77 $\mathrm{mm}$; mean male SVL $=46.84 \mathrm{~mm}(n=33, \mathrm{SD}=4.31)$, mean female $S V L=66.55 \mathrm{~mm}$ $(n=3, \mathrm{SD}=5.44)$. Females are significantly larger than males $(t=7.66, \mathrm{df}=33, P<$ 0.001). A MANOVA on the residuals of the regressions between SVL and the other measured variables indicates lack of significant differences between sexes in size-free morphometry $(F=0.239, \mathrm{df}=17, P=0.060)$.

Color in life. Based on digital photograph of adult male QCAZ 48744 (Fig. 7 $\mathrm{C}-\mathrm{D})$ : dorsum green with irregular light and dark brown marks; canthal region green with cream subocular mark and olive green diffuse band along the posterior half of upper lip; tympanum light brown; flanks light green with dark brown reticulation anteriorly and irregular dark brown blotches posteriorly; dorsal surfaces of thighs, shanks and forelimbs green with transversal brown bands; venter brown with irregular dark brown and cream marks; iris bronze with diffuse brown mid-horizontal line and black reticulations.

Based on digital photograph of juvenile QCAZ 40859 (Fig. 7 F): dorsum green with dark brown marks; upper lip cream with transversal brown bars; flanks light green with brown marks; dorsal surfaces of arms, thighs and shanks green with brown transversal bars; external edge of tarsus with white tubercles; iris bronze with black reticulations and diffuse mid-horizontal dark band between the pupil and posterior border of iris.

In life the Peruvian specimens have extensive blue coloration in the groins, concealed surfaces of thighs and tibia, dorsal surfaces of tarsus, armpits and posterior surfaces of arms (e.g., CORBIDI 10534; Fig. 7 G-H). The iris is highly variable from 

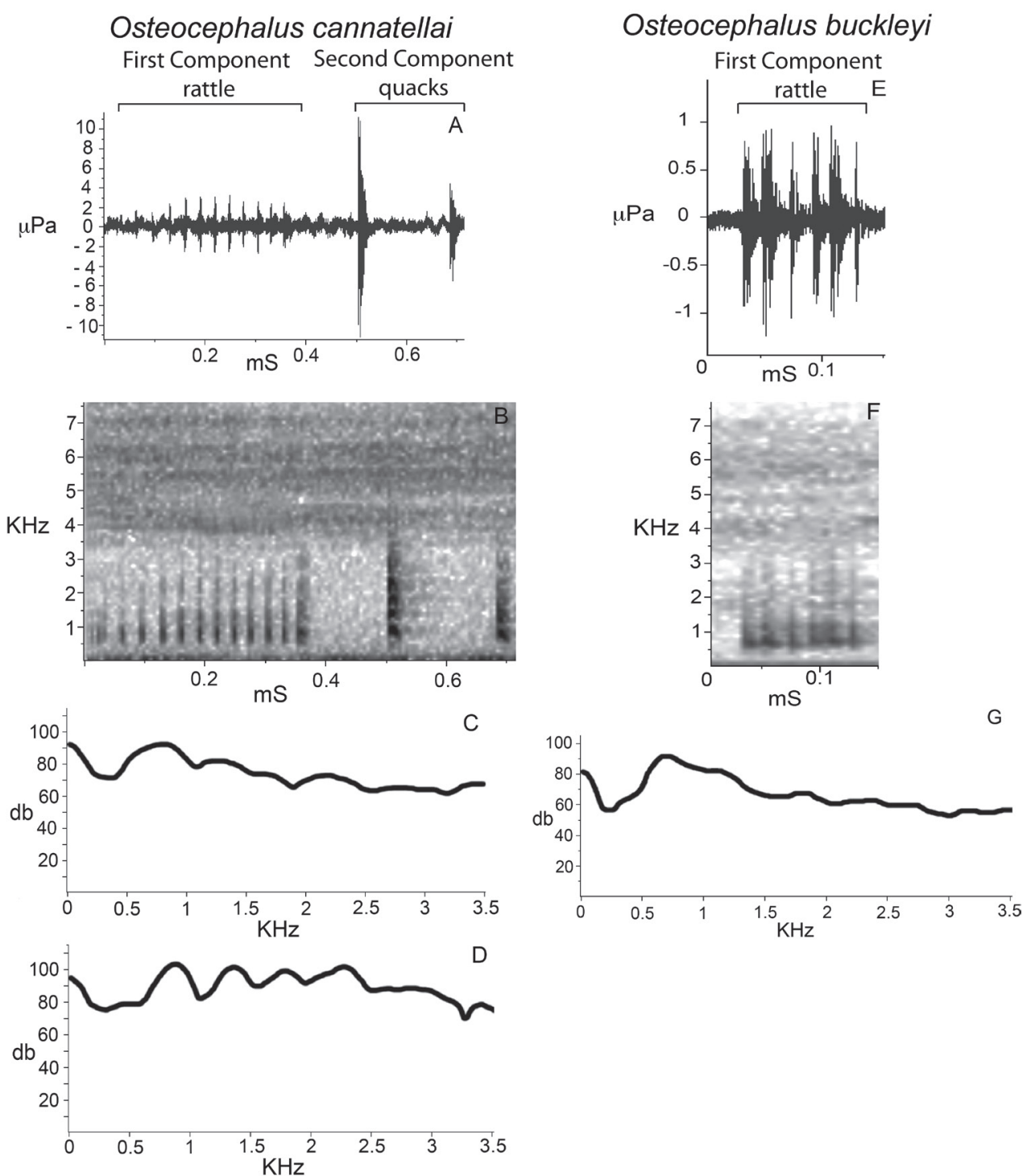

Figure II. Advertisement calls of Osteocephalus. A-D O. cannatellai (QCAZ8322) from Río Piraña, Provincia Orellana, Ecuador; E-G O. buckleyi, from Jatun Sacha, Provincia Napo, Ecuador. A and E are oscillograms, $\mathbf{B}$ and $\mathbf{F}$ spectrograms, $\mathbf{C}$ and $\mathbf{G}$ power spectra of complete call, and $\mathbf{D}$ power spectra of quacks (second component).

light cream to brownish cream and dark brown (CORBIDI 09394); there are always black reticulations and a diffuse mid-horizontal dark band. Some individuals have a diffuse vertical dark band below the pupil.

Green coloration in life changes to cream in preserved specimens.

Call. Males call from vegetation next to rivers or streams. Acoustic parameters of the advertisement call of $O$. cannatellai are shown in Table 4. The call consists of two 
Table 4. Descriptive statistics for call parameters of Osteocephalus buckleyi and O. cannatellai sp. n. Mean $\pm \mathrm{SD}$ is given with range below. The calls of both species have an obligatory first component consistent of a rattle-like note. Osteocephalus cannatellai has a facultative second component consistent of one to three quack notes. Sample sizes are number of males. Temporal characters are shown in seconds; spectral characters in Hertz.

\begin{tabular}{|c|c|c|c|c|}
\hline & \multicolumn{3}{|c|}{ O. cannatellai } & \multirow{2}{*}{$\begin{array}{c}\text { O. buckleyi } \\
\text { Jatun Sacha } \\
(\mathrm{n}=2)\end{array}$} \\
\hline & $\begin{array}{l}\text { Combined } \\
(\mathbf{n}=5)\end{array}$ & $\begin{array}{l}\text { Río Pirańa } \\
(\mathbf{n}=\mathbf{1})\end{array}$ & $\begin{array}{c}\text { Río Pucayacu } \\
(\mathrm{n}=4)\end{array}$ & \\
\hline $\begin{array}{l}\text { Duration of first } \\
\text { component note }\end{array}$ & $\begin{array}{l}0.425 \pm 0.053 \\
(0.356-0.489)\end{array}$ & 0.356 & $\begin{array}{l}0.442 \pm 0.042 \\
(0.389-0.489)\end{array}$ & $\begin{array}{l}0.059 \pm 0.004 \\
(0.056-0.063)\end{array}$ \\
\hline Call Rate & $\begin{array}{c}0.3066 \pm 0.113 \\
(0.208-0.454)\end{array}$ & 0.454 & $\begin{array}{l}0.269 \pm 0.090 \\
(0.208-0.402)\end{array}$ & $\begin{array}{l}1.524 \pm 0.151 \\
(1.417-1.631) \\
\end{array}$ \\
\hline $\begin{array}{l}\text { First component } \\
\text { interval }\end{array}$ & $\begin{array}{l}4.114 \pm 1.722 \\
(2.142-6.004)\end{array}$ & 2.142 & $\begin{array}{l}4.607 \pm 1.528 \\
(2.568-6.004)\end{array}$ & $\begin{array}{l}0.725 \pm 0.140 \\
(0.625-0.824)\end{array}$ \\
\hline $\begin{array}{l}\text { Dominant } \\
\text { Frequency }\end{array}$ & $\begin{array}{c}1049.54 \pm 247.18 \\
(771.6-1412.6)\end{array}$ & 771.616 & \begin{tabular}{|l|}
$1119.02 \pm 221.99$ \\
$(765.68-1472.26)$ \\
\end{tabular} & $\begin{array}{c}745.66 \pm 0.87 \\
(745.04-746.28)\end{array}$ \\
\hline Number of pulses & $\begin{array}{c}12.213 \pm 1.585 \\
(9.8-14.2)\end{array}$ & 12 & $\begin{array}{c}12.266 \pm 1.825 \\
(9.8-14.2)\end{array}$ & $\begin{array}{c}3.328 \pm 0.181 \\
(3.2-3.457) \\
\end{array}$ \\
\hline Pulse rate & $\begin{array}{c}28.932 \pm 4.095 \\
(23.847-34.016) \\
\end{array}$ & 33.661 & \begin{tabular}{|c|}
$27.749 \pm 3.610$ \\
$(22.004-33.495)$ \\
\end{tabular} & $\begin{array}{c}55.833 \pm 1.565 \\
(41.772-69.893) \\
\end{array}$ \\
\hline $\begin{array}{l}\text { Duration of second } \\
\text { component }\end{array}$ & $\begin{array}{l}0.307 \pm 0.106 \\
(0.216-0.488)\end{array}$ & 0.216 & $\begin{array}{c}0.329 \pm 0.108 \\
(0.25-0.488)\end{array}$ & NA \\
\hline $\begin{array}{l}\text { Duration of second } \\
\text { component note }\end{array}$ & $\begin{array}{l}0.032 \pm 0.004 \\
(0.027-0.037)\end{array}$ & 0.027 & $\begin{array}{l}0.033 \pm 0.004 \\
(0.027-0.037)\end{array}$ & NA \\
\hline $\begin{array}{l}\text { Number of second } \\
\text { component notes }\end{array}$ & $\begin{array}{l}0.866 \pm 0.339 \\
(0.445-1.287)\end{array}$ & 1 & $\begin{array}{l}0.832 \pm 0.381 \\
(0.225-1.439)\end{array}$ & NA \\
\hline Quack rate & $\begin{array}{l}0.140 \pm 0.026 \\
(0.108-0.177)\end{array}$ & 0.108 & $\begin{array}{l}0.148 \pm 0.022 \\
(0.125-0.177)\end{array}$ & NA \\
\hline
\end{tabular}

components. The first is obligatory and consists of one to five rattle-like notes. The second component is facultative and consists of one to three quacks. The first component is pulsed and lacks harmonic structure; the second component has visible harmonics and reaches higher amplitude than the first component (Fig. 11).

The advertisement calls of $O$. cannatellai differ markedly from those of $O$. buckleyi. Calls of $O$. buckleyi (Fig. 11) consist of a pulsed rattle-like note repeated at irregular intervals and without a second component. Those calls have a shorter duration, higher repetition rate, and fewer pulses than calls of $O$. cannatellai.

Distribution and ecology. Osteocephalus cannatellai has been recorded at twelve localities, all of them south of the Napo river, in the Ecuadorian and Peruvian Amazon basin (Provincias Morona Santiago, Napo, Orellana, Pastaza, Zamora-Chinchipe, and Datem del Marañón; Fig. 2). Localities with known elevation (El Edén, Huino, Yachana, Zanjarajuno, Río Maderoyacu, Hola Vida, Bobonaza, Nuevo Israel, Yawi, and Kampankis) have a range between 200 and $1290 \mathrm{~m}$ above sea level. Maximum airline distance between localities is $531 \mathrm{~km}$. Osteocephalus cannatellai occurs sympatrically with $O$. buckleyi at Reserva Yachana and with $O$. fuscifacies and $O$. mutabor at Río Pucayacu, Nuevo Israel and Hola Vida. 


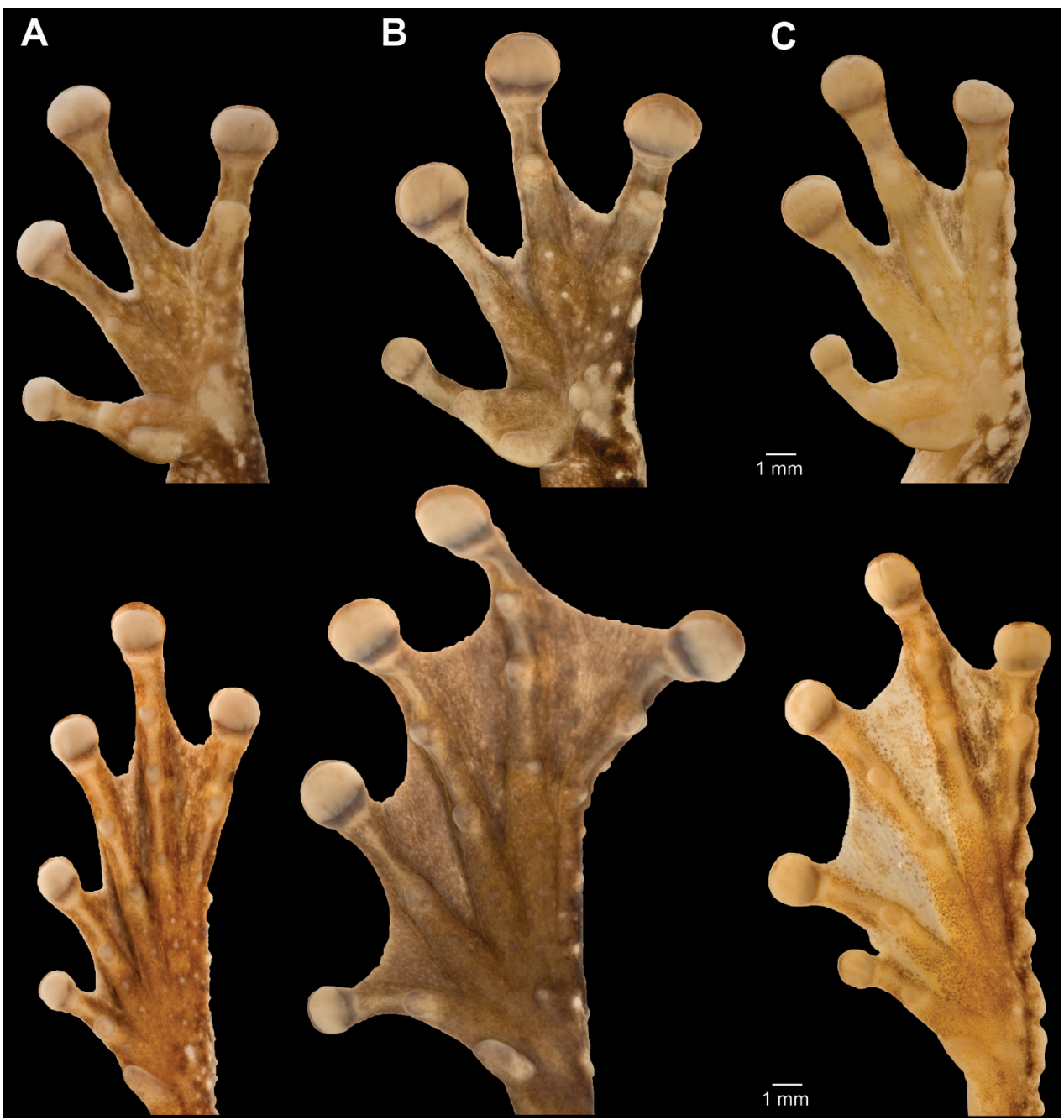

Figure I2. Ventral views of the left hand and foot of Osteocephalus. A Osteocephalus buckleyi (Tarangaro, Ecuador, SVL = $39.8 \mathrm{~mm}$, QCAZ 39191) B O. cannatellai (Zanjarajuno, Ecuador, SVL = $45.32 \mathrm{~mm}$, QCAZ 45907) and C O. cabrerai (Cuyabeno, Ecuador, SVL = $41.62 \mathrm{~mm}$, EPN-H 7204). Hand and foot are shown at the same scale.

Most specimens were collected at Río Pucayacu, a river surrounded by a mixture of primary and secondary forest. Frogs were found next to the river, perching over broad leaves or on tree branches 50 to $230 \mathrm{~cm}$ above the ground. At the collection site, the river has an average width of approximately $10 \mathrm{~m}$, fast running water, and a rocky bottom. Males were calling next to the river between June 26 and July 32010 . Several adults and a juvenile were found on a small stream, tributary of Río Rivadeneira, surrounded by secondary forest, near Río Pucayacu, in March 2008.

Vegetation types (according to the classification of Sierra et al. 1999) are: (1) Amazonian Mountain Range Evergreen Foothill Forest, characterized by a mixture of 

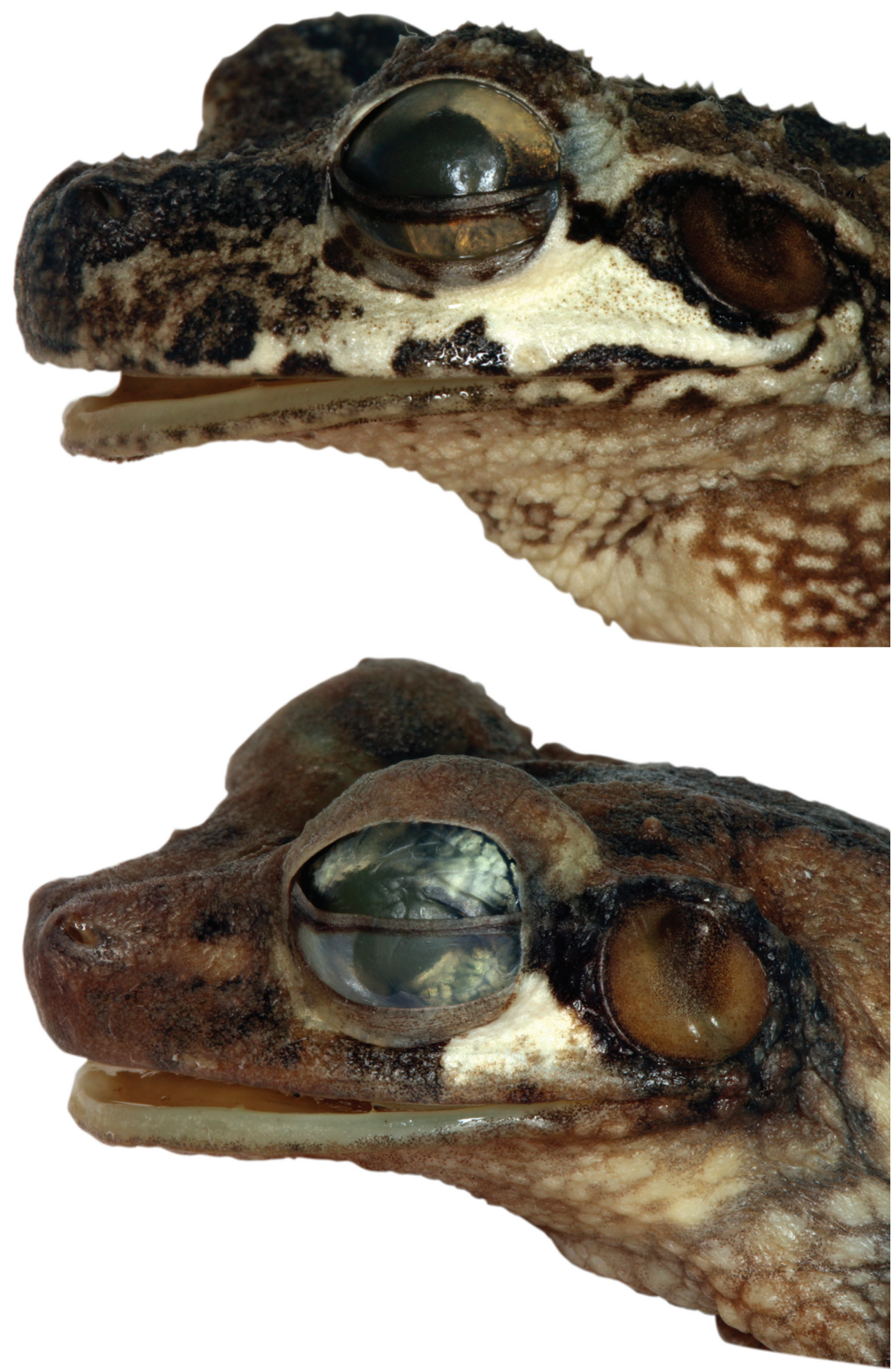

Figure 13. Lateral view of the head of the holotypes of Osteocephalus germani (above CORBIDI 05462) and O. vilmae (below CORBIDI 04773). 
Amazonian and Andean vegetation with a canopy of $30 \mathrm{~m}$ (Río Pucayacu, Bobonaza, and Yawi), (2) Amazonian Lowland Evergreen Forests, characterized by high plant alpha-diversity and a canopy of $30 \mathrm{~m}$ with emergent trees that reach $40 \mathrm{~m}$ (Huino, Río Maderoyacu, Reserva Yachana), and (3) Amazonian Lower Montane Evergreen Forest, with an elevational range of 1300 to $2000 \mathrm{~m}$ above sea level, its canopy can reach 25 to $30 \mathrm{~m}$ (Nuevo Israel; Sierra et al. 1999, Cerón et al. 1999).

Specimens from Peru were collected in Cordillera de Kampankis within an elevational range of 300 to $365 \mathrm{~m}$ above sea level in tall, closed-canopy forest on low hills with well-drained soils at the base of the mountains. The soils have variable proportions of silt, clay and sand, but there are some small patches of sandy soil and limestone outcrops. The forest canopy is about $30 \mathrm{~m}$ tall, with emergent trees reaching $45 \mathrm{~m}$. All individuals were collected in riparian vegetation of low-velocity and low-volume streams with rounded slate rocks lining the stream bed. Some individual were found on leafs of dense populations of rheophytic plants or shrubby Pitcairnia aphelandriflora (Bromeliaceae). Other individuals were found on branches of bushes between 50 and $200 \mathrm{~cm}$ above the ground. Other arboreal frogs at the site were O. mutabor, Hypsiboas cinerascens, and Gastrotheca longipes.

\section{Osteocephalus germani sp. n.}

urn:lsid:zoobank.org:act:556B14DE-AA7D-4112-9C1B-D2814A7D6351

http://species-id.net/wiki/Osteocephalus_germani

Holotype. (Fig. 13, 14) CORBIDI 05462, adult male from Peru, Región Cusco, Provincia La Convención, near Pongo de Mainique in the vicinity of Santuario Natural Megantoni $\left(12.2581^{\circ} \mathrm{S}, 72.8425^{\circ} \mathrm{W}\right), 670 \mathrm{~m}$ above sea level, collected by G. Chavez on 23 April 2010.

Paratopotypes. (Fig. 15 A, C) CORBIDI 06633, adult female, and CORBIDI 06660, adult male, collected with the holotype; CORBIDI 05505, adult female, collected by G. Chavez on 8 November 2009.

Paratypes. (Fig. 15 B, D) Peru: Provincia La Convención, Comunidad Nativa Poyentimari $\left(12.18853^{\circ} \mathrm{S}, 73.00092^{\circ} \mathrm{W}\right), 725 \mathrm{~m}$ above sea level, CORBIDI 08267, 08284, adult females, collected by G. Chavez and D. Vasquez on 28 November 2010; Comunidad Nativa Chokoriari $\left(11.9569^{\circ} \mathrm{S}, 72.9410^{\circ} \mathrm{W}\right), 434 \mathrm{~m}$ above sea level, CORBIDI 08059, adult female, collected by D. Vasquez on 8 December 2010.

Diagnosis. Throughout this section, coloration refers to preserved specimens unless otherwise noted. Osteocephalus germani is a medium-sized species of Osteocephalus having the following combination of characters: (1) size sexually dimorphic; maximum SVL in males $41.45 \mathrm{~mm}(n=2)$, in females $50.76(n=2)$; (2) skin on dorsum bearing tubercles in males, smooth in females; (3) skin on flanks areolate; (4) hand webbing formula varying from I basal II2- $-3^{-}$III $2 \frac{1}{2} 2-2$ IV to I basal II2-3III3- $-3^{-}$IV; foot

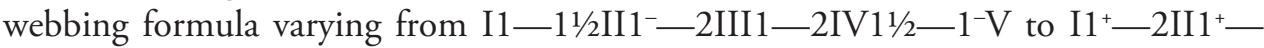
$2 \mathrm{III}^{+}+2{ }^{+} \mathrm{IV} 2-1 \mathrm{~V} ;(5)$ dorsum varying from brown with dark brown marks to light 

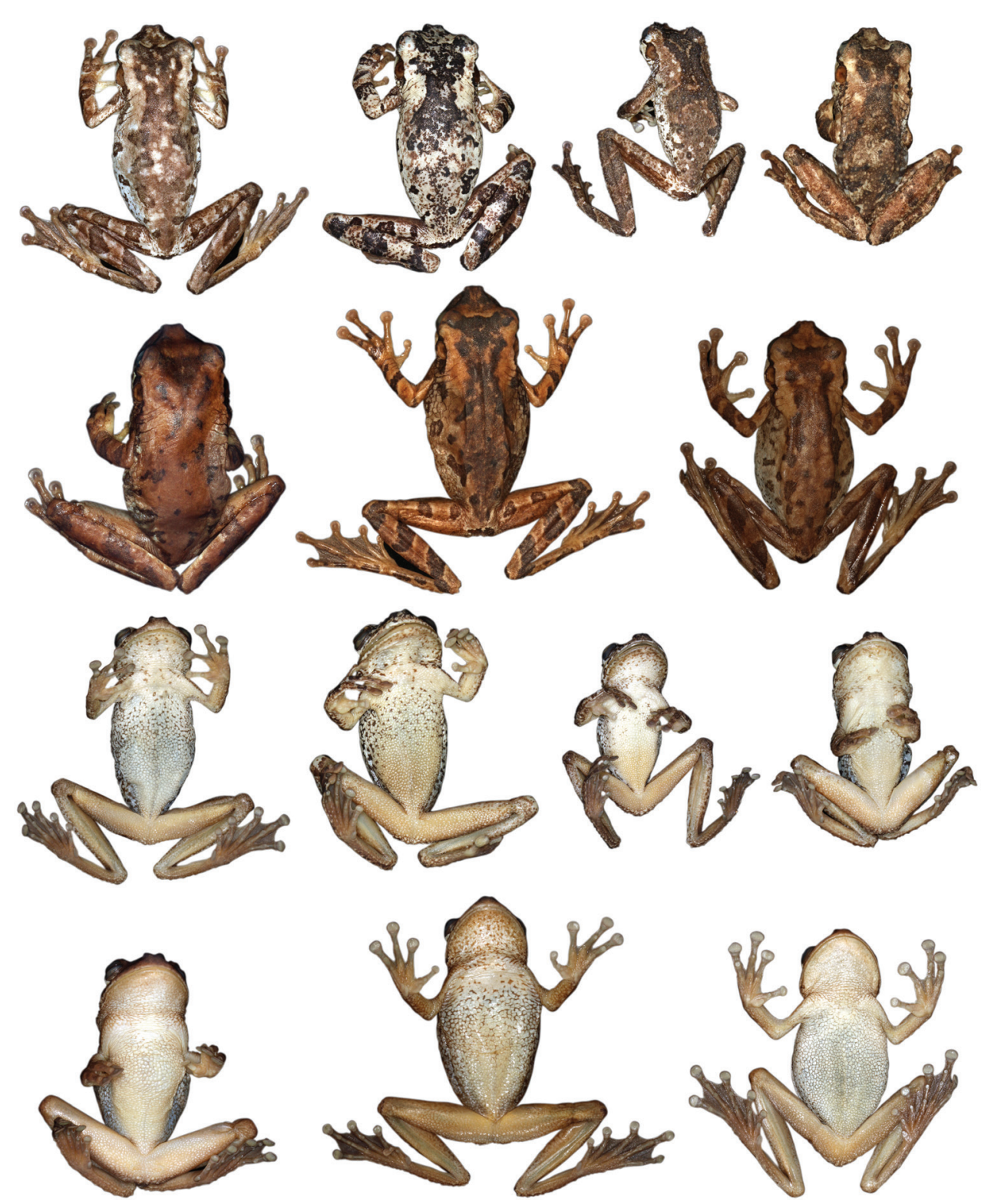

Figure 14. Adult Osteocephalus germani showing variation in dorsal and ventral coloration of preserved specimens. Left to right, upper row: CORBIDI 08267 (female), 05505 (female), 05462 (male, holotype), 06660 (male), CORBIDI 06663 (female), 08284 (female), 08059 (female); third and fourth rows show ventral views of the same specimens, in the same order as in the first two rows. Peru, Region Cusco.

gray with dark brown marks; (6) venter light cream with or without dark brown flecks; (7) cream suborbital mark present, clear labial stripe absent; (8) flanks cream to brownish cream with dark brown blotches and flecks; (9) dermal roofing bones of the skull weakly exostosed; (10) bones green in life; (11) in life, iris golden to reddish golden 
with fine dark reticulation; (12) paired vocal sacs, located laterally, behind jaw articulation, (13) juvenile coloration unknown; (14) larvae unknown.

Osteocephalus germani is most similar to O. buckleyi, O. cabrerai, O. cannatellai sp. n., and $O$. vilmae sp. n. It differs from all of them in lacking prominent tarsal tubercles (tubercles are indistinct in $O$. germani). It further differs from O. buckleyi, $O$. cannatellai, and $O$. vilmae in having a white to light cream venter with or without dark brown flecks (cream with brown speckling in most $O$. buckleyi and O. vilmae, light gray to dark brown in O. cannatellai). Osteocephalus germani also differs from $O$. cannatellai and $O$. vilmae in having more abundant and keratinized dorsal tubercles (dorsal tubercles are scattered and weakly keratinized in O. cannatellai and O. vilmae) and smaller size $(O$. germani male SVL range $=41.26-41.45, n=2$; O. cannatellai male SVL range $=38.46-57.21, n=33$; $O$. vilmae male $S V L$ range $=48.23-55.77, n=5$ ). Mitochondrial DNA sequences show that $O$. germani is the sister species of $O$. cabrerai (Fig. 1). Osteocephalus germani can be easily distinguished from $O$. cabrerai by $(O$. cabrerai in parenthesis): (1) absence of prominent tubercles on the lower jaw (present), (2) smooth outer edge of Finger IV (outer edge with fringe), (3) row of inconspicuous tubercles in the outer edge of tarsus (conspicuous), and (4) less webbing in the hands (in O. germani web reaches the antepenultimate tubercle of Finger IV, in O. cabrerai it reaches the proximal border of the ultimate tubercle; Figs 12 and 16). Osteocephalus germani differs from other species of Osteocephalus in having a combination of a dark golden to tan golden iris, a row of indistinct tubercles in the tarsus, and areolate skin in the flanks. A golden iris with black reticulations further distinguishes $O$. germani from $O$. deridens, O. oophagus, $O$. planiceps, and $O$. taurinus which have bronze to golden irises with black lines radiating from the pupil; iris coloration also differs in $O$. carri, $O$. festae, O. heyeri, O. subtilis, and O. verruciger which have predominantly dark irises, and in O. leoniae which have a bicolor iris (Jungfer 2010; Jungfer and Lehr 2001; Lynch 2002). Osteocephalus germani differs from O. exophthalmus, O. fuscifacies and $O$. leoniae in having abundant keratinized dorsal tubercles in males (tubercles are absent in the three last species). Skin texture in the flanks distinguishes $O$. germani (areolate) from O. mutabor (smooth). Osteocephalus inframaculatus differs from O. germani in coloration of the ventral surfaces of hindlimbs (bold brown blotches in $O$. inframaculatus are absent in O. germani; Jungfer 2010).

Description of holotype. Adult male, $41.26 \mathrm{~mm} \mathrm{SVL}$, head length 12.79, head width 14.23 , eye diameter 5.23 , tympanum diameter 3.79 , femur length 22.3 , tibia length 23.1, foot length 17.97. Head narrower than body, slightly wider than long; snout rounded in dorsal view and truncate in lateral view; distance from nostril to eye longer than diameter of eye; canthus rostralis distinct and straight; loreal region concave; internarial area depressed; nostrils moderately protuberant, directed laterally; interorbital area with tiny keratinized conical tubercles, lateral margins of frontoparietals inconspicuous through skin; eye large, strongly protuberant; tympanic membrane clearly evident, large, slightly wider than high, about two thirds of eye diameter, separated from eye by ca. $85 \%$ of its diameter; tympanic annulus distinct except dorsally where it is covered by supratympanic fold; posterior end of supratympanic fold reaches 


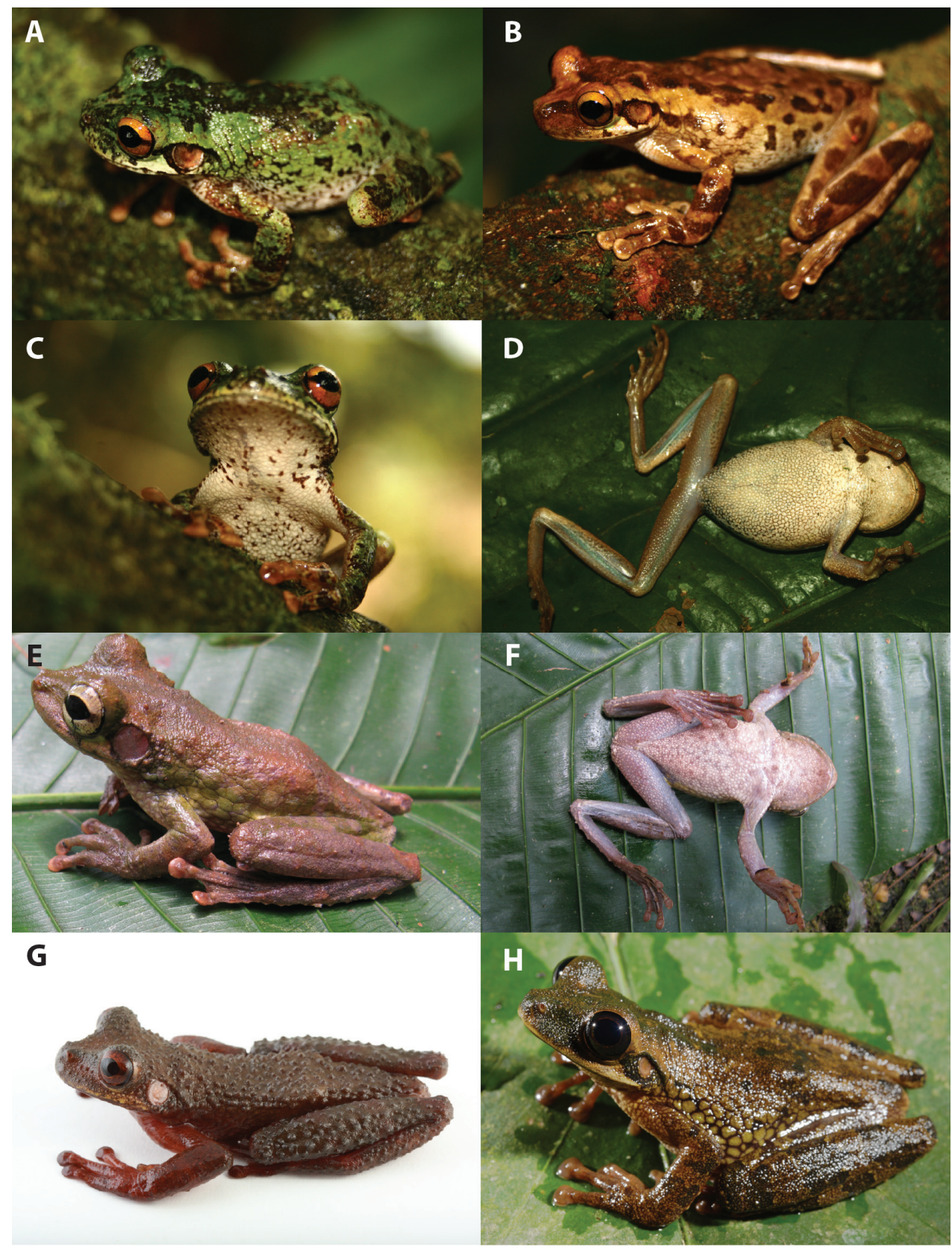

Figure 15. Dorsolateral, frontal, and ventral views of Osteocephalus. A, C Osteocephalus germani, CORBIDI 05505, adult female, SVL $=49.16 \mathrm{~mm}$, Pongo de Mainique, Peru B, D O. germani, CORBIDI 08284, adult female, SVL $=49.00 \mathrm{~mm}$, Comunidad Nativa Poyentimari, Peru E-F O. vilmae CORBIDI 04773 (holotype), adult male, SVL $=51.85 \mathrm{~mm}$, Pampa Hermosa, Peru G Osteocephalus verruciger, QCAZ 41115, adult male, $52.37 \mathrm{~mm}$, Pacto Sumaco, Ecuador H Osteocephalus festae, QCAZ 39801, adult female, SVL $=51.54 \mathrm{~mm}$, Río Napinaza, Ecuador. Photographs A-D by G. Chavez, and E-F by V. Durán. 
arm insertion. Arm slender, axillary membrane present, reaching less than one third of arm length; four small low tubercles present along ventrolateral edge of forearm; relative length of fingers $\mathrm{I}<\mathrm{II}<\mathrm{IV}<\mathrm{III}$; fingers bearing large, oval discs, that of third finger about three fourths of tympanum diameter; subarticular tubercles prominent, round to ovoid except for bifid distal subarticular tubercle of Finger IV; supernumerary tubercles present; palmar tubercle small, elongated; prepollical tubercle large, flat, elliptical; prepollex enlarged; large dark keratinous nuptial excrescences covering inner surface of prepollex up to two thirds the distance between subarticular tubercle and proximal border of disk of thumb; webbing absent between fingers I and II; webbing formula of fingers II2- -3 III $2^{1 / 2}-3^{-}$IV. Small tubercles on tibiotarsal articulation; dorsal surface of tarsus covered by tiny keratinized conical tubercles, more abundant on outer edge; minute tubercles scattered along ventrolateral edge of foot; toes bearing discs slightly wider than long, smaller than those of fingers; relative length of toes $\mathrm{I}<\mathrm{II}<\mathrm{V}<\mathrm{III}<\mathrm{IV}$; outer metatarsal tubercle ill defined, small, round; inner metatarsal tubercle low, ovoid; subarticular tubercles single, round, protuberant; supernumerary tubercles restricted to the soles; webbing formula of toes I1-2II $1-2 \mathrm{III}^{+}-2 \mathrm{IV} 2^{-}-1 \mathrm{~V}$. Skin on dorsum, head, and dorsal surfaces of limbs shagreen covered by conical tubercles with keratinized tips, tiny on head and limbs; skin on flanks areolate; skin on venter coarsely granular; skin on ventral surfaces of head and thighs granular, those of shanks smooth. Cloacal opening directed posteriorly at upper level of thighs; short simple cloacal sheath covering cloacal opening; round tubercles below vent; two conspicuous white tubercles ventrolateral to vent at lower level of thighs. Tongue cordiform, widely attached to floor of mouth; dentigerous processes of the vomers angular, adjacent medially, posteromedial to choanae, bearing 5 and 6 (left/right) vomerine teeth; choanae trapezoidal, oblique; vocal slits moderately long, extending diagonally from lateral end of tongue toward to the angle of snout; vocal sac indistinct above the arm and below the ear.

Color of holotype in preservative. Dorsum light brown with dark brown peripheral marks; dark brown transversal bar between orbits with fine pale borders; dorsal surfaces of forearms grayish brown with dark brown marks, dorsal surfaces of thighs, shanks, and feet grayish brown with diffuse brown transversal bars. Venter light cream with dark brown flecks on the throat and thoracic region and absent on posterior half of the body; ventral surfaces of hindlimbs and forelimbs dirty cream with dark brown flecks on the lateral borders of shanks; outer half of ventral surfaces of forearms dirty cream; sides of head brown with white subocular band extending, below tympanum, two little brown blotches below the eye; tympanic membrane dark brown and area in the periphery of tympanum light brown dorsally and grayish brown behind the tympanum; flanks grayish white, areolate region with dark brown reticulation and flecks. Iris silver with dark brown mid-horizontal line and thin black reticulations.

Color in life. Dorsum brown with irregular dark brown marks; flanks brownish cream with dark brown spots and flecks; dorsal surfaces of thighs, shanks, and forelimbs brown with transversal dark brown bands. Venter whitish cream with brown flecks in throat; ventral surfaces of thighs tan. Iris bronze with thin black reticulations (G. Chávez field notes April 2010). 
Etymology. The new species is dedicated to our colleague German Chávez (CORBIDI), one of the best friends of PJV, for his contributions to Peruvian herpetology and collecting the type series and tissues of this new species.

Variation. Variation in dorsal and ventral coloration of preserved specimens is shown in Figure 14. Dorsal background coloration varies from light brown to light gray; irregular dark brown marks are always present. In females, the dorsum lacks tubercles while in males tubercles are present. The single male paratype (CORBIDI 06660) differs from the holotype in having non-keratinized tubercles.

Ventral surfaces of preserved specimens (Fig. 14) are whitish cream. All the specimens have scattered dark brown flecks on the anterior half of the venter. Ventrally, limbs vary from whitish cream to tan; scant white tubercles can be present in the external edge of the forearm of males (e.g., CORBIDI 06660). The vent region is light brown or dark. Flanks are whitish cream to light gray, areolate in the anterior half and nearly smooth posteriorly. The areolate portion is completely covered by dark brown reticulation and flecks.

Snout is truncate in lateral view except for a female with rounded snout (CORBIDI 06633). Lateral head coloration varies from dull brown (CORBIDI 06633) to cream with dark brown blotches (CORBIDI 05505). The tympanic annulus is concealed dorsally and has lighter color than the background. The distal subarticular tubercle on Finger IV is bifid in all the specimens.

Adult morphometric data are summarized in Table 3. In the examined series, the largest male has a SVL of $41.45 \mathrm{~mm}$ and the largest female $50.76 \mathrm{~mm}$; mean male SVL $=41.35 \mathrm{~mm}(n=2, \mathrm{SD}=0.13)$, mean female $\mathrm{SVL}=49.96 \mathrm{~mm}(n=2, \mathrm{SD}=1.13)$.

Color in life. Based on digital photograph of adult male CORBIDI 06660: dorsum brown with irregular dark brown marks and some scattered light green blotches; canthal region greenish brown with greenish cream subocular mark and dark labial bars; tympanum light brown; flanks light green with dark brown reticulation and dark brown blotches posteriorly; dorsal surfaces of thighs, shanks, and forelimbs brown with transversal dark brown bands and scattered light green blotches. Iris bronze with brown horizontal midline and thin black reticulations.

Based on digital photograph of adult female CORBIDI 06633: dorsum brown with few scattered irregular dark brown marks; canthal region dark brown with greenish cream subocular mark speckled by three small dark brown blotches; tympanum light brown; flanks light brown with dark brown blotches; ventrolateral region cream with fine dark reticulation; dorsal surfaces of thighs, shanks, and forelimbs brown with transversal dark brown bands. Anterior half of venter whitish cream with fine brown reticulation in throat and chest; posterior half of venter and ventral surfaces of thighs tan; iris bronze with diffuse brown mid-horizontal line and thin black reticulations.

Based on digital photograph of adult female CORBIDI 05505 (Fig. 15): dorsum green with irregular dark brown marks; canthal region green with brown mottling and white subocular mark extending to the lips as a white labial stripe along posterior half of the jaw; tympanum light brown; flanks white with dark brown reticulation and small dark brown blotches posteriorly; dorsal surfaces of thighs, shanks, and forelimbs green with transversal dark brown bands and flecks. Venter white with scattered brown 
flecks on throat and chest. Iris reddish gold with diffuse brown mid-horizontal line and thin black reticulations. Based on digital photograph of adult female CORBIDI 08284 (Fig. 15): dorsum light brown with irregular dark brown marks; canthal region brown and greenish white subocular mark; tympanum light brown; flanks light brown with small dark brown blotches; dorsal surfaces of thighs, shanks, and forelimbs light brown with transversal dark brown bands. Venter dull cream. Iris bronze with diffuse brown mid-horizontal line and thin black reticulations.

Distribution and ecology. Osteocephalus germani is known from three localities in southern Peru (Fig. 6). Pongo de Manique and Comunidad Nativa de Poyentimari are in premontane forest on the Upper Urubamba River basin (vegetation types according to ONERN 1976) in the Amazonian foothills of the southern Peruvian Andes, at elevations of 670-725 m; Comunidad Nativa de Chokoriari is Terra Firme Amazonian lowland forests on the lower Urubamba River basin in the southern Peruvian Amazon lowlands, at elevation of $434 \mathrm{~m}$. In Pongo de Mainique the new species was found close to rocky streams in low-hill primary forest with arboreal ferns and abundant epiphytes. At this locality, O. germani was sympatric with $O$. castaneicola and $O$. mimeticus. In Comunidad Nativa de Poyentimari, $O$. german $i$ was found close to rocky streams in a step area of very wet high-hill primary forest with abundant ferns (including arboreal), epiphytes, lichens and mosses. At this locality the new species was sympatric with O. mimeticus. In Comunidad Nativa de Chokoriari, O. germani was found close to a black-water slow-running creek in a patch of secondary forest, surrounded by pastures for cattle and plantations. The forest was dominated by bamboo and Cecropia spp. and the creek had sandy soils covered by leaf litter. No other species of Osteocephalus were found in this locality.

All specimens were collected next to temporary pools, perching over broad leaves or on tree branches 100 to $200 \mathrm{~cm}$ above the ground. Many streams surround the collection sites.

Remarks. In the phylogeny (Fig. 1), two specimens from gen bank (EF376030 from French Guiana and AY843705 from Río Jurua, Brazil) are grouped with O. germani in a strongly supported clade $(\mathrm{PP}=0.96)$ and are likely conspecific or represent one or two closely related species. The specimen from French Guiana was reported as "O. oophagus" by Salducci et al. (2002, 2005); the specimen from Brazil was reported as "O. cabrerai" by Faivovich et al. (2005). Both individuals appear to be misidentified.

\section{Osteocephalus vilmae sp. $\mathrm{n}$.}

urn:lsid:zoobank.org:act:681AAC6A-8710-4276-AA79-BD1F5C58C1DC http://species-id.net/wiki/Osteocephalus_vilmae

Holotype. (Figs 13 and 15) CORBIDI 04773, adult male from Peru, Region Loreto, Provincia Datem del Marañón, Pampa Hermosa (3.0650 $0^{\circ}$, $\left.75.8264^{\circ} \mathrm{W}\right), 200 \mathrm{~m}$ above sea level, collected by V. Duran on 28 March 2008.

Paratypes. Five adult males: Ecuador: Provincia de Orellana: Pompeya-Iro road, km 80, Río Beye, QCAZ 51205, collected by E. Toral, I. G. Tapia, T. Camacho, and S. 


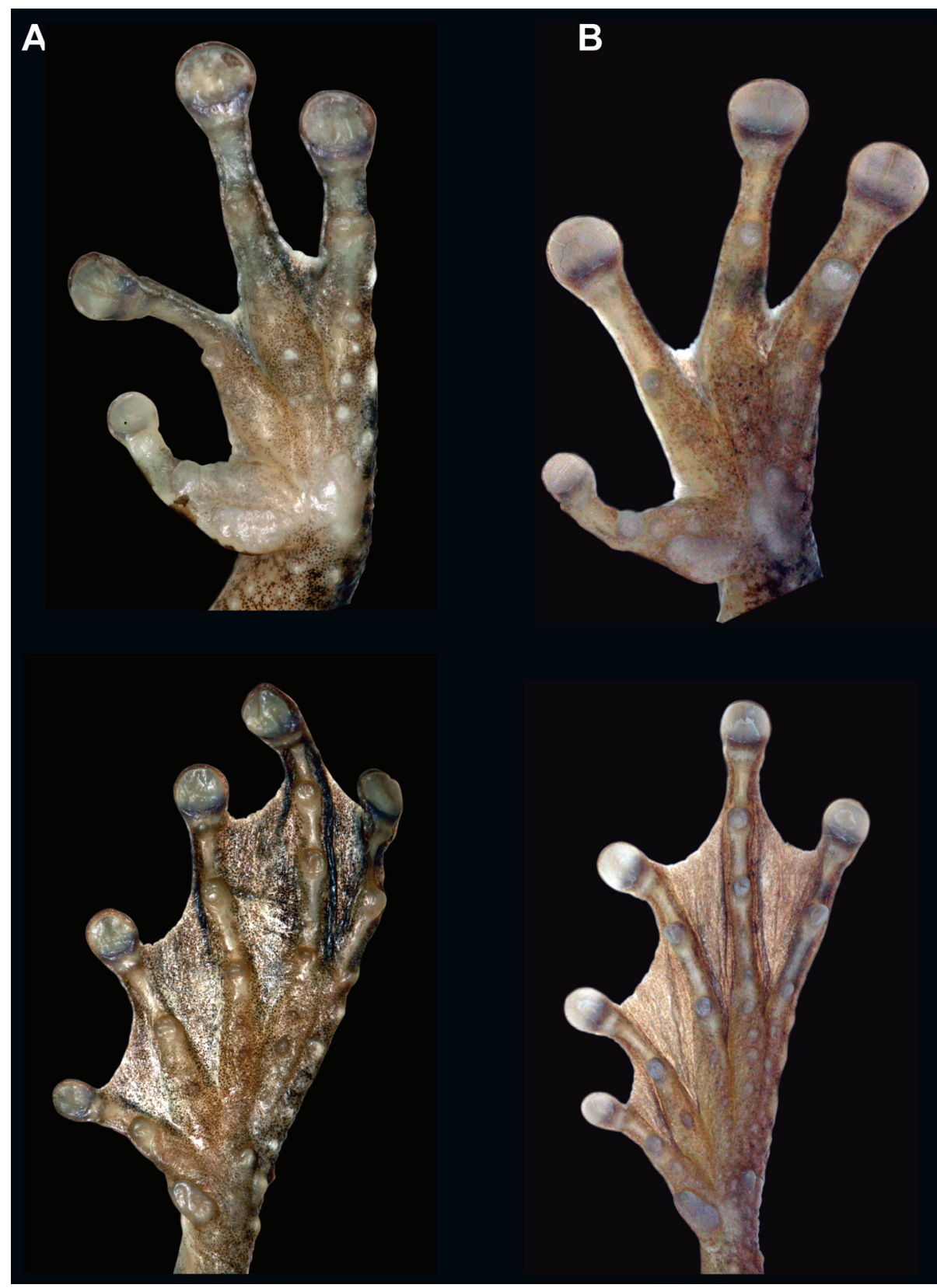

Figure 16. Ventral views of left hand and foot of Osteocephalus vilmae and O. germani. A O. vilmae (Jibarito, Peru, SVL = $48.31 \mathrm{~mm}$, CORBIDI 06469), and B O. germani (Comunidad Nativa Poyentimari, Peru, SVL $=49.00 \mathrm{~mm}$, COBIDI 08284).

R. Ron on 31 May 2011; Provincia Pastaza: Nuevo Corrientes, $250 \mathrm{~m}$ above sea level, QCAZ 14947, collected by F. Villamarín on August 2000. Peru: Provincia Datem del Marañón: Andoas $\left(2.6516^{\circ} \mathrm{S}, 76.5137^{\circ} \mathrm{W}\right), 151 \mathrm{~m}$ above sea level, CORBIDI 01086, 
collected by A. Delgado on September 2008; Jibarito (2.7356 ${ }^{\circ}$ S, $\left.76.0318^{\circ} \mathrm{W}\right), 197$ $\mathrm{m}$ above sea level, CORBIDI 06469, collected by A. Delgado on 14 July; Capihuari Norte $\left(2.6642^{\circ} \mathrm{S}, 76.5012^{\circ} \mathrm{W}\right), 270 \mathrm{~m}$ above sea level, CORBIDI 05031 , collected by J. C. Chaparro on March 2008.

Diagnosis. Throughout this section, coloration refers to preserved specimens unless otherwise noted. Osteocephalus vilmae is a medium-sized species of Osteocephalus having the following combination of characters in males (females are unknown): (1) maximum SVL in males $55.77 \mathrm{~mm}(n=6)$; (2) skin on dorsum bearing few scattered to abundant tubercles; (3) skin on flanks areolate with big flattened warts; (4) hand webbing formula varying from I basal II basal III2- 2 IV to I basal III $1^{2} / 2_{3}{ }^{2}{ }_{3} \mathrm{III} 2^{2} /{ }_{3}-2 \frac{1}{2} \mathrm{IV}$; foot webbing formula varying from $\mathrm{I} 1-1 \frac{1}{1} 2 \mathrm{II} 1-2^{-} \mathrm{III}^{-}-2 \mathrm{IV}^{-}-1^{-} \mathrm{V}$ to I1 ${ }^{+}-2$ III $1-2 \mathrm{III} 1^{+}-$ $2^{+}$IV2- $1^{+} \mathrm{V}$ (Fig. 16); (5) dorsum varying from light brown with dark brown marks to light gray with dark brown marks; (6) venter varying from light gray to tan with lighter dots and/or dark brown blotches; (7) cream suborbital mark present, clear labial stripe absent; (8) flanks cream with darker reticulations and dark marks; (9) dermal roofing bones of the skull weakly exostosed; (10) in life, bones green; (11) in life, iris light cream to dirty cream with irregular reticulations; (12) paired vocal sacs small, located laterally, behind jaw articulation, (13) juveniles unknown; (14) larvae unknown.

Osteocephalus vilmae is most similar to O. buckleyi and O. cannatellai. It differs from $O$. buckleyi in having (1) scattered and weakly keratinized dorsal tubercles (abundant and keratinized in O. buckleyi), (2) larger size (O. vilmae mean male $S V L=55.77$, $\mathrm{SD}=3.17, n=5 ;$ O. buckleyi mean male $\mathrm{SVL}=41.12, \mathrm{SD}=2.49, n=24$; differences are significant: $t=6.50, P<0.001$; Fig. 5), and (3) more extensive and conspicuous areolate area on flanks (from axillary region to groin, with big flattened warts, in $O$. vilmae; restricted to anterior one half of flank in O. buckleyi). The range of genetic distances (uncorrected $p$ for gen $12 \mathrm{~S}$ ) between $O$. vilmae and $O$ buckleyi is 0.9 to $1.6 \%$. Both species are sympatric at $\mathrm{km} 80$ Pompeya-Iro road indicating the existence of reproductive barriers between them.

Osteocephalus vilmae differs from $O$. cannatellai in having a larger tympanum $(-1 / 4$ of head length in $O$. vilmae vs. $-1 / 5$ in $O$. cannatellai), and areolate flanks with big flattened warts (areolate with small flattened warts in $O$. cannatellai). Mitochondrial DNA sequences show that $O$. vilmae and $O$. cannatellai are not sister species (Fig. 1). Osteocephalus vilmae differs from O. cabrerai in (1) lacking prominent tubercles on the lower jaw, (2) having smooth outer edge of Finger IV (outer edge with fringe in O. cabrerai), (3) having less webbing in the hands (in O. vilmae webbing reaches two thirds of the distance between the ultimate and penultimate tubercle of Finger IV, in O. cabrerai it reaches the proximal border of the ultimate tubercle; Figs 12 and 16), and (4) low to indistinct tubercles in the tarsus (prominent in O. cabrerai).

A cream to bronze iris with black reticulations distinguishes $O$. vilmae from $O$. deridens, $O$. oophagus, $O$. planiceps, and $O$. taurinus which have bronze to golden irises with black lines radiating from the pupil; iris coloration also differs from O. carri, O. festae, $O$. heyeri, $O$. subtilis, and $O$. verruciger which have predominantly dark irises, and from $O$. leoniae which have a bicolor iris (Jungfer 2010; Jungfer and Lehr 2001; Lynch 2002). 
Osteocephalus vilmae is larger than O. exophthalmus (maximum male SVL in O. vilmae $55.77 \mathrm{~mm}, n=5$; in O. exophthalmus $32.7 \mathrm{~mm}, n=3$; Smith and Noonan 2001) and O. fuscifacies (maximum SVL $=44.17, n=21$ ). Skin texture in the flanks distinguishes $O$. vilmae (coarsely areolate) from $O$. mutabor and O. yasuni (smooth). Osteocephalus inframaculatus differs from $O$. vilmae in coloration of the ventral surfaces of hindlimbs (bold brown blotches in O. inframaculatus are absent in O. vilmae; Jungfer 2010).

Description of holotype. Adult male, $51.85 \mathrm{~mm}$ SVL, head length 18.9, head width 19.0, eye diameter 6.8, tympanum diameter 4.9, femur length 28.0, tibia length 28.7, foot length 22.1. Head narrower than body, nearly as wide as long; snout truncate in lateral and dorsal views; distance from nostril to eye longer than diameter of eye; canthus rostralis distinct and straight; loreal region concave; internarial area depressed; nostrils moderately protuberant, directed laterally; interorbital area flat, lateral margins of frontoparietals distinct through skin; eye large, strongly protuberant; tympanic membrane clearly evident, slightly wider than high, about two thirds of eye length, separated from eye by ca. $85 \%$ of its diameter; tympanic annulus distinct except dorsally where it is covered by supratympanic fold; posterior end of supratympanic fold reaches mid arm insertion. Arm slender, axillary membrane present, reaching one third of arm length; three small low tubercles present along ventrolateral edge of forearm; relative length of fingers $\mathbf{I}<\mathbf{I I}<\mathbf{I V}<\mathbf{I I I}$; fingers bearing large, oval discs, that of third finger about three fourths of tympanum diameter; subarticular tubercles prominent, round to ovoid, bifid

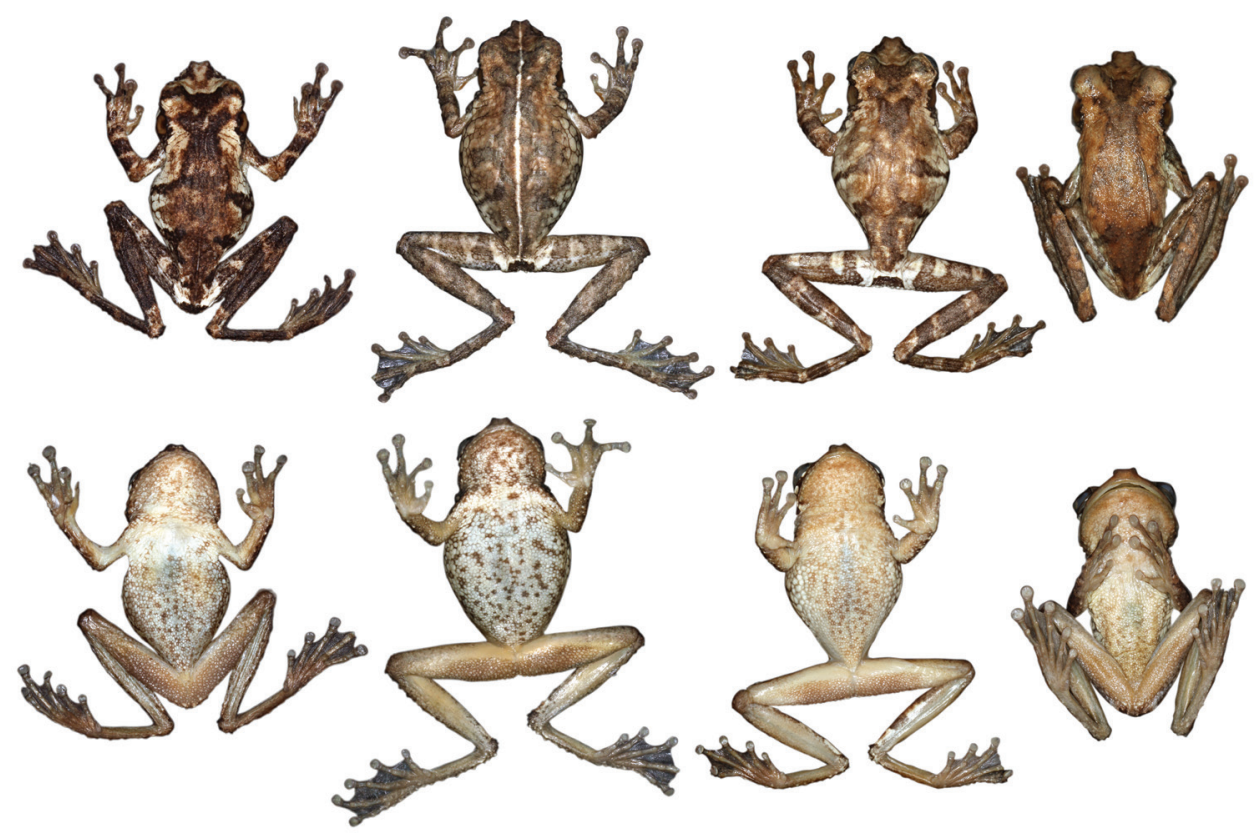

Figure 17. Adult male Osteocephalus vilmae showing variation in dorsal and ventral coloration of preserved specimens. Upper row, from left to right: CORBIDI 5031, CORBIDI 6469, CORBIDI 1086, CORBIDI 4773 (holotype), Peru, Región Loreto, Provincia Datem del Marañón, Jibarito, Capihuari Norte, Andoas, Pampa Hermosa. 
in distal subarticular tubercle of Finger IV; supernumerary tubercles present; palmar tubercle small, elongated; prepollical tubercle large, flat, elliptical; prepollex enlarged; large dark keratinous nuptial excrescences covering inner surface of prepollex almost reaching the proximal border of disk of thumb; webbing basal between fingers I and II; webbing

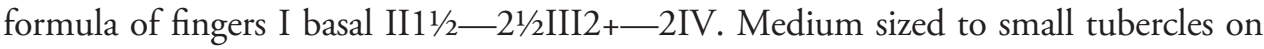
tibiotarsal articulation; scattered low tubercles on tarsus, more abundant on outer edge; small tubercles scattered along ventrolateral edge of foot; toes bearing discs slightly wider than long, smaller than those of fingers; relative length of toes $\mathrm{I}<\mathrm{II}<\mathrm{V}<\mathrm{III}<\mathrm{IV}$; outer metatarsal tubercle ill defined, small, round; inner metatarsal tubercle large, ovoid; subarticular tubercles single, round, protuberant; supernumerary tubercles restricted to the soles; webbing formula of toes I1-2-II1-2III1-2IV2-1-V. Skin on dorsum, head, and dorsal surfaces of limbs shagreen, with scattered tubercles; minute keratinized conical tubercles present on the eyelids and dorsal surface of head; skin on flanks areolate with big flattened warts; skin on venter coarsely granular; skin on ventral surfaces of head and thighs granular, that on shanks smooth. Cloacal opening directed posteriorly at upper level of thighs; short simple cloacal sheath covering cloacal opening; round tubercles below vent; two distinct white tubercles ventrolateral to vent. Tongue cordiform, widely attached to floor of mouth; dentigerous processes of the vomers angular, adjacent medially, posteromedial to choanae, bearing 9 and 6 (left/right) vomerine teeth; choanae trapezoidal, oblique; vocal slits short and curved posteroventral to the angle of snout at the base of tongue; vocal sac barely distinct above the arm and below the ear.

Color of holotype in preservative. Dorsum brown with a single diffuse interorbital mark; dorsal surfaces of forearms brown with diffuse brown bands; dorsal surfaces of hindlimbs brown with diffuse dark brown marks on shanks and feet. Venter dirty cream with light brown spots, more abundant on posterior half of the body; ventral surfaces of hindlimbs and forelimbs dirty cream without marks but with distinct white tubercles on forearms; outer half of ventral surfaces of forearms dark brown; sides of head light brown with oblique white bar from posteroventral border of orbit to border of jaw, below tympanum; vertical diffuse brown bar below eye, anterior to white bar; area behind white bar and eye dark brown including periphery of tympanum; flanks dirty cream, areolate region with brown reticulation. Iris silver with a brown mid-horizontal line and thin black reticulations.

Color of holotype in life. Based on digital photograph (Fig. 15). Dorsum pale brown without marks; canthal region pale brown with diffuse pale green subocular mark and dark stripe along the posterior half of upper lip; tympanum pink; flanks light green without marks; dorsal surfaces of thighs and tarsus pale brown with greenish brown transversal bands, forearms greenish brown; tibia pale brown without marks; anterior and posterior surfaces of thighs, concealed surfaces of tibia, and metatarsus pale blue. Venter dirty cream with light brown spots, more abundant on posterior half of the body; ventral surfaces of hindlimbs and forelimbs dirty cream. Iris dirty cream with brown transversal midline and black reticulations.

Etymology. The specific name is a patronym for Vilma Duran, in recognition of her continued work and efforts toward the improvement of the herpetological collection of CORBIDI and also for collecting the holotype and tissue of this new species. 
Variation. Dorsal and ventral coloration of preserved specimens is shown in Figure 17. Dorsal background coloration varies from light brown to brown; irregular dark brown or dark gray marks are always present (Fig. 17). Flanks are always cream to grayish cream. Two specimens have a cream middorsal line from the tip of the snout to the vent (CORBIDI 06469, QCAZ 51205). The prominence of the tubercles can decrease in preserved specimens: when collected, CORBIDI 01086 had large conspicuous dorsal tubercle, in preservative tubercles are barely noticeable.

Ventral surfaces of preserved specimens (Fig. 17) vary from cream to vanilla. In most specimens, there are dark brown spots, more distinct posteriorly or in the throat (e.g., CORBIDI 06469); ventrally, limbs vary from dirty cream to light brown; all specimens have small white tubercles in the external edge of the forearm. The vent region is gray to brown with dark brown flecks or dots. Flanks are cream to gray, areolate, with dark brown reticulations, dots, and blotches along the entire flank or restricted to the posterior half (e.g. CORBIDI 05031).

Head shape is truncate in dorsal view and truncate in lateral view. Lateral head coloration varies from light brown with dark mottling (CORBIDI 01086) to grayish white with dark brown canthus rostralis and preocular stripes (CORBIDI 05031). All specimens have a white to cream subocular mark. The tympanic annulus is concealed dorsally and has lighter color than the background. The distal subarticular tubercle on Finger IV is bifid in all specimens.

Adult morphometric data are summarized in Table 3. In the examined series, the largest male has a SVL of $55.77 \mathrm{~mm}$; mean male SVL $=50.74 \mathrm{~mm}(n=6, \mathrm{SD}=3.17)$.

Color in life. Based on a digital photograph of adult male CORBIDI 01086: dorsum light brown with irregular dark brown and light green marks; canthal region greenish brown with white subocular mark and dark brown band along posterior half of upper lip; tympanum pink contrasting with dark brown tympanic annulus; flanks light green with dark brown reticulation anteriorly and few irregular dark brown blotches posteriorly; dorsal surfaces of thighs, shanks and forelimbs brown with dark brown transversal bands; posterior surfaces of thighs light green; venter white speckled with light brown blotches; iris light cream with brown mid-horizontal line and fine black reticulations.

Distribution and ecology. Osteocephalus vilmae is know from seven localities in the Peruvian and Ecuadorian Amazon basin (northern Loreto region), four at Río Corrientes (Jibarito, Nuevo Corrientes, Pampa Hermosa, and Shiviyacu), two near Rio Pastaza in the border Ecuador-Peru (Andoas and Capahuari Norte) and one at Provincia de Orellana, Pompeya-Iro road (Fig. 2). The elevations of these localities are between 150 to $270 \mathrm{~m}$ above sea level. Maximum airline distance between localities is $158 \mathrm{~km}$. The Peruvian localities are dominated by Terra Firme forest. Specimens collected in Capahuari Norte were found in a stream surrounded by a mixture of primary and secondary forest. In Jibarito, Pampa Hermosa, and Shiviyacu the frogs were found in primary forest in a swamp close to a stream. All specimens were next to the streams, perching on tree branches 100 to 200 $\mathrm{cm}$ above the ground. Osteocephalus vilmae occurs sympatrically with $O$. buckleyi at $\mathrm{km} 80$ Pompeya-Iro road. At the Peruvian localities it co-occurs with O. mutabor and O. planiceps. 
Table 5. Character loadings and eigenvalues for Principal Components (PC) I-III. The analysis was based on seven morphometric variables of adult Osteocephalus buckleyi, O. cabrerai, O. cannatellai sp. n., $O$. festae, O. germani sp. n., O. verruciger and O. vilmae sp. n. Bold figures indicate highest loadings.

\begin{tabular}{l|c|c|c|c|c|c}
\hline \multirow{2}{*}{ Variable } & \multicolumn{3}{|c|}{ PCA Males } & \multicolumn{3}{c}{ PCA Females } \\
\cline { 2 - 7 } & PC I & PC II & PC III & PC I & PC II & PC III \\
\hline Femur length & $\mathbf{0 . 5 5 7}$ & 0.126 & 0.052 & $\mathbf{0 . 5 5 3}$ & 0.030 & 0.193 \\
\hline Foot length & 0.425 & -0.400 & 0.046 & 0.451 & 0.374 & -0.106 \\
\hline Head length & 0.106 & 0.459 & $\mathbf{0 . 7 1 5}$ & -0.004 & -0.326 & $\mathbf{0 . 6 4 9}$ \\
\hline Head width & 0.433 & -0.053 & -0.480 & 0.161 & $\mathbf{0 . 6 5 2}$ & -0.083 \\
\hline Eye diameter & 0.173 & $\mathbf{0 . 5 5 4}$ & -0.332 & -0.018 & 0.349 & $\mathbf{0 . 6 9 0}$ \\
\hline Tympanum diameter & 0.066 & $\mathbf{0 . 5 4 0}$ & -0.233 & -0.376 & 0.421 & 0.201 \\
\hline Tibia length & $\mathbf{0 . 5 2 5}$ & -0.108 & 0.298 & $\mathbf{0 . 5 6 8}$ & -0.164 & 0.080 \\
\hline Eigenvalue & 2.451 & 1.432 & 1.083 & 2.311 & 1.768 & 1.222 \\
\hline
\end{tabular}
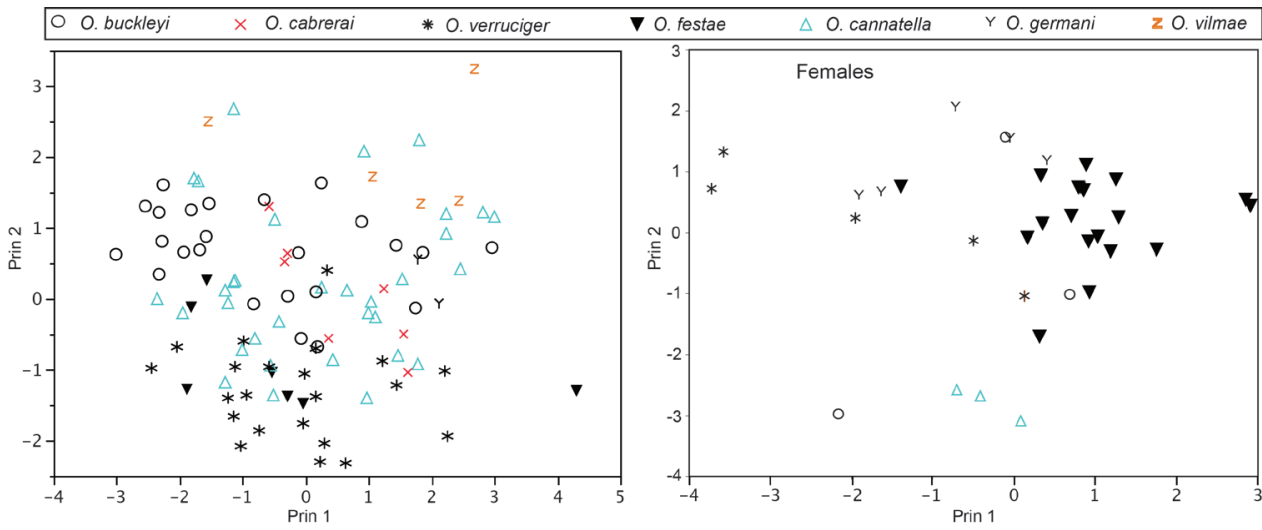

Figure 18. Principal components from analysis of seven size-corrected morphological variables. See Table 5 for character loadings on each component.

\section{Morphometric comparisons among species}

Three components with eigenvalues $>1.0$, accounting for $70.9 \%$ of the total variation, were extracted from the PCA for males (Table 5). The highest loadings were femur length and tibia length for PC I, eye diameter and tympanum diameter for PC II, and head length for PC III (Table 5). Some species pairs have at least partly segregating morphometric spaces: O. festae-O. buckleyi, O. festae-O. cabrerai, O. vilmae-O.buckleyi, and $O$. vilmae-O. cabrerai (Fig. 18). Osteocephalus germani does not overlap with $O$. vilmae, $O$. festae and $O$. cabrerai but this differentiation requires to be verified with larger sample sizes for $O$. germani (currently $n=2$ ). Principal Component I mainly describes hindlimb length (Table 5). There is low interspecies differentiation along PC I. Species with low scores on PC II are O. festae and O. verruciger; O. vilmae has high scores. Pairwise comparisons between $O$. festae and all other species (except $O$. 
verruciger) are significant (all $P$ values for $t$ tests $<0.04$ ); $O$. verruciger also shows significant differences with all the remaining species (all $P$ values $<0.004$ ).

Three components with eigenvalues $>1.0$ were extracted from the PCA for females (Table 5). The three components accounted for $75.7 \%$ of the total variation. The highest loadings for the PCA for females were tibia length and femur length for PC I, head width for PC II, and eye diameter and head length for PC III (Table 5). As in the PCA for males, there is wide overlap in morphometric space among species (Fig. 18). The only exception is $O$. cannatellai, which segregates from the other species along PC II . However, larger sample sizes are required to confirm this differentiation.

In the DFA classification on males, all O. cabrerai, O. festae, O. germani, O. verruciger, and $O$. vilmae were correctly classified $(n=7,7,2,22$, and 5 respectively). In $O$. buckleyi, 17 out of 24 specimens were correctly classified ( 4 were misclassified as $O$. cabrerai, 2 as $O$. germani, and 1 as $O$. vilmae); in O. cannatellai, only 4 out of 33 specimens were incorrectly classified, 2 as $O$. buckleyi and 2 as $O$. vilmae. Overall, the DFA show morphometric differentiation among the analyzed species. The DFA on females shows even better discrimination because all individuals were correctly assigned to their own species.

\section{Discussion}

Similarly to previous studies on Amazonian amphibians (e.g., Elmer et al. 2007; Fouquet et al. 2007; Funk et al. 2011) our results document a large proportion (300\% increase) of hidden diversity within a set of populations that were previously treated as a single widely distributed species. Moreover, because most of our sampling was restricted to Ecuador and Peru, it is likely that there are even more species than found in our study. These results highlight the need to carry out large-scale genetic surveys of Amazonian amphibians to achieve a more realistic understanding of their diversity and evolution.

Genetic evidence is a valuable taxonomic tool but, in most cases, is insufficient to define species boundaries without reference to other sets of characters like advertisement calls or external morphology. Taxonomic reviews of Amazonian amphibians suggest that morphological characters are too conservative to define species boundaries because closely related species share similar morphology (e.g., Elmer et al. 2007; Fouquet et al. 2012; Funk et al. 2011; Lougheed et al. 2006; Padial et al. 2009). Our results, however, indicate that in some groups, like the $O$. buckleyi species complex, this is not necessarily the case. The three new species described here are diagnosable with morphological evidence alone and are distinctive from the other species of the complex. Morphological differences are also evident between O. buckleyi, O. cabrerai, O. carri, $O$. festae, and $O$. mutabor. Thus, none of the species of the complex are strictly cryptic (i.e., all of them can be identified using morphological characters) although their diagnosis based on morphology is challenging. Other groups of Amazonian amphibians on which phylogenetic analyses of DNA have led to the discovery of species that turned out to be morphologically distinct are the Hypsiboas fasciatus-calcaratus complex (Funk et al. 2011) and the Pristimantis "ockendeni" complex (Elmer and Cannatella 2008). 
We suspect that the difficulty in defining species boundaries based on morphology arises from the high intraspecific polymorphism in coloration characteristic of most groups of dull-colored Amazonian amphibians like Osteocephalus and Pristimantis (see for example Fig. 2 in Elmer and Cannatella 2008 and Figs 3, 8, 14, 17 herein). If this is the case, understanding the evolutionary processes that generate and maintain polymorphism in coloration could help to predict which Amazonian taxa are more likely to contain "cryptic" diversity. One plausible process is frequency dependent predation which, occurs when the probability of predation is inversely correlated to the frequency of a given prey type in the population (for a review see Punzalan et al. 2005). Under this scenario, predators use search images to find preys and are better at detecting previously seen prey types because they have learned to find them. Although other processes could also explain polymorphisms (e.g., deferential selection associated with spatial variation in backgrounds), the available evidence suggests that some form of frequency dependent selection is the most likely explanation for color polymorphism in anurans (Milstead et al. 1974; Wells 2007, pp. 715).

Most Osteocephalus have a predominantly and highly polymorphic brown coloration and are cryptic against the background where they are found by day (Deichmann 2008; Deichmann and Williamson 2007; SRR pers. obs.) If polymorphisms are an adaptation to avoid falling into search categories of visually oriented predators, the difficulties of species delimitation based on morphological characters could be a byproduct of this selective pressure. This hypothesis needs to be tested empirically because if verified it could help to understand why several groups of Neotropical amphibians contain a large proportion of cryptic species.

\section{Biogeography and speciation}

Examination of the geographic ranges of sister species can provide insights into modes of speciation. Our phylogeny of the $O$. buckleyi species complex recovered four sister species pairs of which one is sympatric (O. buckleyi-O. vilmae) and three are allopatric. Among the allopatric pairs, two involve a lowland species sister to a highland species. Osteocephalus mutabor occurs at lower altitudes (range 230-1240 m) than its sister species, O. festae (860$2383 \mathrm{~m})$. Similarly, O. cannatellai has a lower distribution $(200-1290 \mathrm{~m})$ than its sister species, O. verruciger (950-2120 m). Because most species of Osteocephalus are restricted to elevations below $1000 \mathrm{~m}$, the distributions of $O$. festae and $O$. verruciger probably represent parallel and recent colonization events from the lowlands. This geographic pattern suggests that speciation has been a result of ecological mediated selection along an altitudinal gradient. Interestingly, both highland species resemble each other closely in external morphology (Figs 15 and 18) suggesting convergence as a byproduct of adaptation to similar environments. Speciation associated with ecological divergence along altitudinal gradients was also reported by Graham et al. (2004) in dendrobatid frogs and more recently by Salerno et al. (2012) between Tepuihyla and its sister lowland species, "Osteocephalus" exophthalmus.

At the intraspecific level, we found low genetic divergence with the only exception of Osteocephalus festae (up to $2.8 \%$ of uncorrected $p$ distance in gene $12 \mathrm{~S}$ ). We also 
found a concordant geographic pattern of divergence in O. buckleyi and O. mutabor because in both the most divergent population was the most northern of them, in the Cuyabeno region. High divergence of samples from Cuyabeno relative to others to the south was also reported for Pristimantis kichwarum (Elmer and Cannatella 2008). Samples of O. mutabor, O. buckleyi (sensu stricto) and O. cannatellai show genetic structure generally congruent with geography (i.e., geographically close localities tend to be genetically similar). Overall, our intraspecific sampling reveals low levels of genetic differentiation and genetic variation geographically structured.

\section{Acknowledgments}

This study was supported by grants from the Secretaría Nacional de Educación Superior, Ciencia, Tecnología e Innovación de Ecuador SENESCYT (PI-C08-0000470) and Dirección General Académica of Pontificia Universidad Católica del Ecuador. For the loan of specimens and access to collections we are indebted to A. Almendáriz and Barry Clarke. Santiago Castroviejo y J. M. Padial provided tissues of Colombian $O$. cabrerai. William E. Duellman shared information on type material. Ministerio de Ambiente of Ecuador and the Dirección General Forestal y Fauna Silvestre (DGFFS) of Peru issued collection permits. PJVs fieldwork in northern Loreto was part of the Rapid Biological Inventories led by the Field Museum, Chicago. PJV is grateful to Programa de Monitoreo Biológico COGA, Transportadora de Gas del Peru (TGP), and Knight Piesold Consultores S.A. for support of the fieldwork where Osteocephalus germani were collected. We are indebted to A. Catenazzi, A. Delgado, C. Landauro, G. Chavez, J. C. Chaparro, M. Cuyos, V. Duran, J. Delia, D. Acosta-López, S. AldásAlarcón, A. Almendáriz, L. Bustamante, L. A. Coloma, P. Peña-Loyola, N. Peñafiel, D. Almeida-Reinoso, J. Brito-Molina, K. Elmer, S. North, D. Salarzar, I. G. Tapia, and R. Tarvin for providing specimens and photos. Centro Científico Zanjarajuno and Estación Científica Yasuní of Universidad Católica del Ecuador provided accommodation during fieldwork. Daniel Moen kindly gave advice on laboratory protocols. Rebecca Tarvin and P. Peña-Loyola provided audio recordings of O. cannatellai. Diego Paucar made a drawing of the holotype and P. Santiana helped to prepare illustrations. Open access to this paper was supported by the Encyclopedia of Life (EOL) Open Access Support Project (EOASP).

\section{References}

Bass MS, Finer M, Jenkins CN, Kreft H, Cisneros-Heredia DF, McCracken SF, Pitman NC, English PH, Swing K, Villa G, Di Fiore A, Voigt CC, Kunz TH (2010) Global conservation significance of Ecuador's Yasuni National Park. Plos One 5: e8767. doi: 10.1371/journal.pone.0008767 Boulenger GA (1882) Catalogue of the Batrachia Salientia. Ecaudata in the Collection of the British Museum. British Museum, London. 
Brandley MC, Schmitz A, Reeder TW (2005) Partitioned Bayesian analyses, partition choice, and the phylogenetic relationships of scincid lizards. Systematic Biology 54: 373-390. doi: 10.1080/10635150590946808

Cerón C, Palacios W, Valencia R, Sierra R (1999) Las formaciones naturales de la Costa del Ecuador. In: Sierra R (Ed) Propuesta preliminar de un sistema de clasificación de vegetación para el Ecuador continental. Proyecto INEFAN/GERF-BIRF y Ecociencia, Quito.

Charif RA, Clark CW, Fristrup KM (2004) Raven 1.2 User's Manual. Cornell Laboratory of Ornitology, Ithaca, NY, USA.

Cochran DM, Goin CJ (1970) Frogs of Colombia. United States National Museum Bulletin 288: 1-655. doi: 10.5479/si.03629236.288.1

Crump ML (1974) Reproductive strategies in a Tropical Anuran Community. Miscellaneous Publications Museum of Natural History University of Kansas 61: 1-68.

Darst CR, Cannatella DC (2004) Novel relationships among hyloid frogs inferred from 12S and 16S mitochondrial DNA sequences. Molecular Phylogenetics and Evolution 31: 462-475. doi: 10.1016/j.ympev.2003.09.003

Deichmann JL (2008) Osteocephalus oophagus (NCN). Terrestrial behavior. Herpetological Review 39: 338-339.

Deichmann JL, Williamson GB (2007) Osteocephalus yasuni (NCN) and Osteocephalus planiceps (NCN). Terrestrial behavior. Herpetological Review 38: 189.

Duellman WE (1970) Hylid frogs of Middle America. Monograph of the Museum of Natural History University of Kansas 1: 1-753. doi: 10.5962/bhl.title.2835

Duellman WE, Mendelson JR (1995) Amphibians and reptiles from nortern Departamento Loreto, Peru: taxonomy and biogeography. University of Kansas Science Bulletin 55: 329-376.

Edwards SV, Liu L, Pearl DK (2007) High-resolution species trees without concatenation. Proceedings of the National Academy of Sciences of the United States of America 104: 5936-4941. doi: 10.1073/pnas.0607004104

Elmer KR, Cannatella DC (2008) Three new species of leaflitter frogs from the upper Amazon forests: cryptic diversity within Pristimantis “ockendeni” (Anura: Strabomantidae) in Ecuador. Zootaxa 1784: 11-38.

Elmer KR, Davila JA, Lougheed SC (2007) Cryptic diversity and deep divergence in an upper Amazonian leaflitter frog, Eleutherodactylus ockendeni. BMC Evolutionary Biology 7: 247. doi: 10.1186/1471-2148-7-247

Faivovich J, Haddad CFB, Garcia PCA, Frost DR, Campbell JA, Wheeler WC (2005) Systematic review of the frog family Hylidae, with special reference to Hylinae: phylogenetic analysis and taxonomic revision. Bulletin of the American Museum of Natural History 294: 6-228. doi: 10.1206/0003-0090(2005)294[0001:SROTFF]2.0.CO;2

Fouquet A, Gilles A, Vences M, Marty C, Blanc M, Gemmell NJ (2007) Underestimation of species richness in neotropical frogs revealed by mtDNA analyses. Plos Biology 2: e1109. doi: 10.1371/journal.pone.0001109

Fouquet A, Recoder R, Teixeira M, Cassimiro J, Amaro RC, Camacho A, Damasceno R, Carnaval AC, Moritz C, Rodriguez MT (2012) Molecular phylogeny and morphometric analyses reveal deep divergence between Amazonia and Atlantic forest species of Dendrophryniscus. Molecular Phylogenetics and Evolution 62: 826-838. doi: 10.1016/j.ympev.2011.11.023 
Frost DR (2010) Amphibian Species of the World: an Online Reference v. 5.4. http://research. amnh.org/vz/herpetology/amphibia/ [accessed 8-04-2010]

Funk WC, Caminer M, Ron SR (2011) High levels of cryptic species diversiy uncovered in Amazonian frogs. Proceedings of the Royal Society B-Biological Sciences 279: 1806-1814 doi: $10.1098 / \mathrm{rspb} .2011 .1653$

Gaige HT (1929) Three new tree-frogs from Panama and Bolivia. Occasional Papers of the Museum of Zoology, University of Michigan 207: 1-6.

Goebel AM, Donnelly MA, Atz M (1999) PCR primers and amplification methods for 12S ribosomal DNA, the control region, cytochrome oxidase I, and cytochrome b in bufonids and other frogs, and an overview of PCR rimers which have amplified DNA in amphibians successfully. Molecular Phylogenetics and Evolution 11: 163-199. doi: 10.1006/ mpev. 1998.0538

Graham CH, Ron SR, Santos JC, Schneider CJ, Moritz C (2004) Integrating phylogenetics and environmental niche models to explore speciation mechanisms in dendrobatid frogs. Evolution 58: 1781-1793. doi: 10.1554/03-274

Jungfer KH (2010) The taxonomic status of some spiny-backed treefrogs, genus Osteocephalus (Amphibia: Anura: Hylidae). Zootaxa 2407: 28-50.

Jungfer KH (2011) A new tree frog of the genus Osteocephalus from high altitudes in the Cordillera del Cóndor, Ecuador (Amphibia: Anura: Hylidae). Herpetological Journal 21: 247-253.

Jungfer KH, Hödl W (2002) A new species of Osteocephalus from Ecuador and a redescription of O. leprieurii (Duméril \& Bibron, 1841) (Anura: Hylidae). Amphibia-Reptilia 23: 21-46. doi: $10.1163 / 156853802320877609$

Jungfer KH, Lehr E (2001) A new species of Osteocephalus with bicoloured iris from Pozuzo (Peru: Departamento de Pasco) (Amphibia: Anura: Hylidae). Zoologische Abhandlungen Staatliches Museum für Tierkunde Dresden 19: 321-329.

Jungfer KH, Weygoldt P (1999) Biparental care in the tadpole-feeding Amazonian treefrog Osteocephalus oophagus. Amphibia-Reptilia 20: 235-249. doi: 10.1163/156853899507040

Katoh K, Misawa K, Kuma K, Miyata T (2002) MAFFT: a novel method for rapid multiple sequence alignment based on fast Fourier transform. Nucleic Acids Research 30: 3059-3066. doi: 10.1093/nar/gkf436

Lougheed SC, Austin JD, Bogart JP, Boag PT, Chek AA (2006) Multi-character perspectives on the evolution of intraspecific differentiation in a neotropical hylid frog. BMC Evolutionary Biology 6: 23. doi: 10.1186/1471-2148-6-23

Lynch JD (2002) A new species of the genus Osteocephalus (Hylidae: Anura) from the Western Amazon. Revista de la Academia Colombiana de Ciencias Exactas, Físicas, y Naturales 26: 289-292. Lynch JD (2006) The amphibian fauna in the Villavicencio region of eastern Colombia. Caldasia 28: 135-155.

Lynch JD, Duellman WE (1997) Frogs of the genus Eleutherodactylus in Western Ecuador. Special Publication The University of Kansas Natural History Museum 23: 1-236. doi: 10.5962/bhl.title.7951 
Maddison WP, Maddison DR (2009) Mesquite: a modular system for evolutionary analysis. Version 2.72. http://mesquiteproject.org

Milstead WW, Rand AS, Stewart MM (1974) Polymorphism in cricket frogs: an hypothesis. Evolution 28: 489-491. doi: 10.2307/2407175

Moen DS, Wiens JJ (2009) Phylogenetic evidence for competitively driven divergence: bodysize evolution in Caribbean treefrogs (Hylidae: Osteopilus). Evolution 63: 195-214. doi: 10.1111/j.1558-5646.2008.00538.x

Moravec J, Aparicio J, Guerrero-Reinhard M, Calderón G, Jungfer KH, Gvozdík V (2009) A new species of Osteocephalus (Anura: Hylidae) from Amazonian Bolivia: first evidence of tree frog breeding in fruit capsules of the Brazil nut tree. Zootaxa 2215: 37-54.

Myers CW, Duellman WE (1982) A new species of Hyla from Cerro Colorado, and other tree frog records and geographical notes from Western Panama. American Museum Novitates 2752: 1-32.

ONERN (1976) Mapa Ecológico del Perú. Guía Explicativa. Oficina Nacional de Evaluación de Recursos Naturales (ONERN), Lima.

Padial JM, Castroviejo-Fisher S, Köhler J, Vilá C, Chaparro JC, De la Riva I (2009) Deciphering the products of evolution at the species level: the need for an integrative taxonomy. Zoologica Scripta 38: 431-447. doi: 10.1111/j.1463-6409.2008.00381.x

Padial JM, De la Riva I (2009) Integrative taxonomy reveals cryptic Amazonian species of Pristimantis (Anura: Strabomantidae). Zoological Journal of the Linnean Society 155: 97122. doi: 10.1111/j.1096-3642.2008.00424.x

Peracca MG (1904) Viaggio del Dr. Enrico Festa nell'Ecuador e regioni vicine. Bollettino dei Musei di Zoologia ed Anatomia comparata, Università di Torino 465: 1-41.

Posada D (2008) Phylogenetic model averaging. Molecular Biology and Evolution 25: 12531256. doi: $10.1093 / \mathrm{molbev} / \mathrm{msn} 083$

Punzalan D, Rodd FH, Hughes KA (2005) Perceptual processes and the maintenance of polymorphism through frequency-dependent predation. Evolutionary Ecology 19: 303-320. doi: $10.1007 /$ s10682-005-2777-z

Rambaut A, Drummond AJ (2007) Tracer v1.4. University of Edinburgh. Available from http://beast.bio.ed.ac.uk/Tracer

Ron SR, Santos JC, Cannatella DC (2006) Phylogeny of the túngara frog genus Engystomops (= Physalaemus pustulosus species group; Anura; Leptodactylidae). Molecular Phylogenetics and Evolution 39: 392-403. doi: 10.1016/j.ympev.2005.11.022

Ron SR, Toral E, Venegas PJ, Barnes CW (2010) Taxonomic revision and phylogenetic position of Osteocephalus festae (Anura: Hylidae) with description of its larva. ZooKeys 70: 67-92. doi: 10.3897/zookeys.70.765

Ronquist F, Teslenko M, van der Mark P, Ayres DL, Darling A, Höhna S, Larget B, Liu L, Suchard MA, Huelsenbeck JP (2012) MrBayes 3.2: efficient bayesian phylogentic inference and model choice across a large model space. Systematic Biology 61(3): 539-542. doi: $10.1093 /$ sysbio/sys 1029

Salducci MD, Marty C, Chappaz R, Gilles A (2002) Molecular phylogeny of French Guiana Hylinae: implications for the systematic and biodiversity of the Neotropical frogs. Comptes Rendus Biologies 325: 141-153. doi: 10.1016/S1631-0691(02)01423-3 
Salducci MD, Marty C, Fouquet A, Gilles A (2005) Phylogenetic relationships and biodiversity in Hylids (Anura: Hylidae) from French Guiana. Comptes Rendus Biologies 328: 1009-1024. doi: 10.1016/j.crvi.2005.07.005

Salerno PE, Ron SR, Seńaris JC, Rojas-Runjaic FJM, Noonan BP, Cannatella DC (2012) Ancient Tepui summits harbor young rather than old lineages of endemic frogs. Evolution 66: 3000-3013. doi: 10.1111/j.1558-5646.2012.01666.x

Sambrook J, Fritsch EF, Maniatis T (1989) Molecular Cloning: a Laboratory Manual. Cold Spring Harbor Laboratory Press, New York, USA.

SAS Institute (2008) User guide. Version 8.01. SAS Institute, Cary.

Sierra R, Cerón C, Palacios W, Valencia R (1999) Mapa de vegetación del Ecuador Continental 1:1'000.000. Proyecto INEFAN/GEF-BIRF, Wildlife Conservation Society y Ecociencia, Quito.

Simmons JE (2002) Herpetological collecting and collection management. Herpetological Circular 31: 1-153.

Smith EN, Noonan BP (2001) A new species of Osteocephalus (Anura: Hylidae) from Guyana. Revista de Biologia Tropical 49: 347-357.

Trueb L, Duellman WE (1971) A synopsis of Neotropical hylid frogs, genus Osteocephalus. Occasional Papers of the Museum of Natural History University of Kansas 1: 1-47.

Wells KD (2007) The Ecology and Behavior of Amphibians. The University of Chicago Press, London.

Werner F (1901) Über Reptilien und Batrachier aus Ecuador und Neu-Ginea. Verhandlungen des Zoologisch-Botanischen Vereins in Wien 50: 593-614.

Wiens JJ, Morrill MC (2011) Missing data in phylogenetic analysis: reconciling results from simulations and empirical data. Systematic Biology 60(5): 719-731. doi: 10.1093/sysbio/ syr025

Wiens JJ, Fetzner JW, Parkinson CL, ReederTW (2005) Hylid frog phylogeny and sampling strategies for speciose clades. Systematic Biology 54:778-807. doi: 10.1080/10635150500234625

Wiens JJ, Graham CH, Moen DS, Smith SA, Reeder TW (2006) Evolutionary and ecological causes of the latitudinal diversity gradient in hylid frogs: treefrog trees unearth the roots of high tropical diversity. American Naturalist 168: 579-596. doi: 10.1086/507882

Wiens JJ, Kuczynski CA, Hua X, Moen DS (2010) An expanded phylogeny of treefrogs (Hylidae) based on nuclear and mitochondrial sequence data. Molecular Phylogenetics and Evolution 55: 871-882. doi: 10.1016/j.ympev.2010.03.013 


\section{Appendix I}

Osteocephalus alboguttatus. ECUADOR: PROVINCIA PASTAZA: Canelos (QCAZ 15981).

Osteocephalus buckleyi. ECUADOR: PROVINCIA NAPO: Cando, 700 m (QCAZ 24446-48); Cabañas Pimpilalo, 600 m (QCAZ 23043); Ahuano, 410 m (QCAZ 36703); Jatun Sacha, 420 m (QCAZ 2876, 48093, 48827); Serena, Río Jatunyacu, 520 m (QCAZ 25321); Tena, 550 m (QCAZ 8809-10); Juan Pablo II, Río Punino, 360 m (EPN-H 5476); Santa Rosa de Arapino, 570 m (EPN-H 6209); Nuevo Rocafuerte, Bloque 3, Pozo PCSA 1, 210 m (EPN-H 6516); PROVINCIA ORELLANA: El Edén, 235 m (QCAZ 33148); Río Yasuní (QCAZ 7360); Puente del Río Beque, 228 m (QCAZ 43071); Río Rumiyacu, Parque Nacional Yasuní, 250 m (QCAZ 16007); Taracoa, 251 m (QCAZ 34963); Aguarico, Parque Nacional Yasuní , Pozo Ewa, 320 m (EPN-H 2785-87); Pozo Sunka, 279 m (EPNH 2757-58); Dayuano, Cotapino, 500 m (EPN-H 7471); Guiyero, 248 m (EPNH 10909); Comuna Huatarco, Pozo Chonta, 300 m (EPN-H 11710, 11717-18); PROVINCIA PASTAZA: Canelos, 650 m, (BMNH 1947.2.13.44; lectotype); Tarangaro, 338 m (QCAZ 39073-74, 39081-82, 39146, 39172, 39191); Villano, 380 m (QCAZ 38503, 38704-05, 38713, 39285); Kurintza, 380 m (QCAZ 39033); PROVINCIA SUCUMBÍOS: Tarapoa (QCAZ 14948); Puerto Bolívar, 240 m (QCAZ 28231); Playas de Cuyabeno, 230 m (QCAZ 28277, 28280, 28395); Tarapoa-Puerto Carmen road, bridge over Río Cuyabeno, 290 m (QCAZ 28427); Comuna Shuar Chari, 280 m (EPN-H 4875); Cascales, Pozo Diamante, 410 m (EPN-H 5418, 5475); La Barquilla (EPN-H 7524-26); PERU: REGION LORETO: PROVINCIA DE REQUENA: Sierra del Divisor, $500 \mathrm{~m}$ (CORBIDI 03747); PROVINCIA DATEM DEL MARAÑÓN: Singasapa, 186 m (CORBIDI 06522); Sargento Puño, 206 m (CORBIDI 07458-59, 07462, 07473, 07516, 07558, 07560, 08675, 08714); PROVINCIA DE MAYNAS: Río Momon-San Luis de Vista Alegre (Puchana), 108 m (CORBIDI 2980-81).

Osteocephalus cabrerai. ECUADOR: PROVINCIA SUCUMBÍOS: Cuyabeno, Campamento Concienti, $260 \mathrm{~m}$ (EPN-H 7201-5); Puerto Bolívar, $240 \mathrm{~m}$ (QCAZ 27923, 28231); Campamento Guepicillo, 220 m (CORBIDI 00119-22, 00200); PERU: REGION LORETO: PROVINCIA DE REQUENA: Sierra del Divisor, $500 \mathrm{~m}$ (CORBIDI 02452, 02632, 05064); PROVINCIA DE MAYNAS: Gueppi, 220 m (CORBIDI 00199); Rio Yanayacu, Campamento Curupa, 125 m (CORBIDI 05819-21, 05831).

Osteocephalus deridens. ECUADOR: PROVINCIA ORELLANA: Estación Científica Yasuní, Universidad Católica del Ecuador, Parque Nacional Yasuní (QCAZ 12556).

Osteocephalus festae. ECUADOR: PROVINCIA LOJA: San Francisco, Arco Iris Reserve, Parque Nacional Podocarpus $\left(3.9884^{\circ} \mathrm{S}, 79.0930^{\circ} \mathrm{W}\right), 2200 \mathrm{~m}(\mathrm{QCAZ}$ 39364); PROVINCIA MORONA SANTIAGO: Río Napinaza, $6.6 \mathrm{~km}$ N from General Leonidas Plaza (Limón) in the road to Mendez $\left(2.9266^{\circ} \mathrm{S}, 78.4070^{\circ} \mathrm{W}\right)$, 1010 m (QCAZ 26283, 26304, 26488, 26552, 26561, 32835, 38081, 38420, 
39799, 39804-6, 39798-803, 39808-12); San Carlos, San Miguel and Río Oro, 600-1200 m (QCAZ 11624-26); PROVINCIA ZAMORA CHINCHIPE: Miasí Alto ( $\left.4.2502^{\circ} \mathrm{S}, 78.6174^{\circ} \mathrm{W}\right), 1250-1300 \mathrm{~m}$ (QCAZ 41039); Reserva Tapichalaca $\left(4.5500^{\circ} \mathrm{S}, 79.1291^{\circ} \mathrm{W}\right), 1637 \mathrm{~m}$ (QCAZ 45674); PERU: REGIÓN DE AMAZONAS: PROVINCIA DE BAGUA: Cataratas de Paraiso-Chonza Alta $\left(5.6026^{\circ} \mathrm{S}, 78.398^{\circ} \mathrm{W}\right), 1342 \mathrm{~m}$ (CORBIDI 760-64, 758-59); Camñopite $\left(5.6147^{\circ} \mathrm{S}, 78.3319^{\circ} \mathrm{W}\right), 1650 \mathrm{~m}$ (CORBIDI 1962-65, 2992); PROVINCIA DE BONGARA: Quebrada Goca (on Yambrasbamba road) $\left(5.7641^{\circ} \mathrm{S}, 77.9129^{\circ} \mathrm{W}\right.$ ), 1711 m (CORBIDI 10461); PROVINCIA DE YAMBRASBAMBA: Quebrada Goca (on Yambrasbamba road) $\left(5.7641^{\circ} \mathrm{S}, 77.9129^{\circ} \mathrm{W}\right), 1711 \mathrm{~m}$ (CORBIDI 10461); REGIÓN DE SAN MARTÍN: PROVINCIA MARISCAL CACERES: Río Lejia $\left(6.8365^{\circ} \mathrm{S}, 77.4860^{\circ} \mathrm{W}\right), 1500 \mathrm{~m}$ (CORBIDI 623, 624); PROVINCIA RIOJA: Bajo Naranjillo (5.8157 $\left.{ }^{\circ}, 77.3367^{\circ} \mathrm{W}\right), 844 \mathrm{~m}$ (CORBIDI 3386) ); PROVINCIA LAMAS: Cataratas de Ahuashiyacu $\left(6.4174^{\circ} \mathrm{S}, 76.2893^{\circ} \mathrm{W}\right), 600 \mathrm{~m}$ (CORBIDI 09585, 09587).

Osteocephalus fuscifacies. ECUADOR: PROVINCIA NAPO: El Tena-Talag Road, 15 $\mathrm{km}$ from Tena, $550 \mathrm{~m}$ (QCAZ 8806); PROVINCIA ORELLANA: Pompeya-Iro Road, 38 km SE from Pompeya (QCAZ 8137); Estación Científica Yasuní, Universidad Católica del Ecuador, 240 m (QCAZ 20785).

Osteocephalus leoniae. - PERU: REGIÓN CUSCO: Provincia La Convención: Tangoshiari, 1135 m (CORBIDI 00306); REGIÓN SAN MARTIN: Provincia de Rioja: El Dorado, $844 \mathrm{~m}$ (CORBIDI 01415).

Osteocephalus mutabor. ECUADOR: PROVINCIA MORONA SANTIAGO: Cantón Morona, Nuevo Israel, 1290 m (QCAZ 46470-71); PROVINCIA NAPO: Chontapuntas, Comunidad Sumak Sacha-Pozo Yuralpa Centro 1 (QCAZ 28646-48); Huino, around the waterfall (QCAZ 30916-17, 30919-20, 30922-23, 30925-26); PROVINCIA ORELLANA: km 22 Pompeya-Iro Road, 287 m (QCAZ 42999); PROVINCIA PASTAZA: Pomona, Fundación Hola Vida, 846 m (QCAZ 25603, 25684); Cantón Santa Clara, Río Pucayacu, Colonia Mariscal Sucre (QCAZ 29430, 36935, 36946, 40253); Canelos (QCAZ 41030); PROVINCIA SUCUMBIOS: Puerto Bolívar, $240 \mathrm{~m}$ (QCAZ 28223). PERU: REGION LORETO, Provicia Loreto: Andoas, 187 m, (CORBIDI 04645); REGION AMAZONAS: Cordillera de Kampankis (CORBIDI 09369).

Osteocephalus planiceps. ECUADOR: PROVINCIA DE NAPO: Chontapuntas, Comunidad Sumak Sacha-Pozo Yuralpa Centro 1 (QCAZ 28648); PROVINCIA ORELLANA: Parque Nacional Yasuní, km 38 Pompeya-Iro Road, 280 m (QCAZ 5134, 14842); Estación Científica Yasuní, Universidad Católica del Ecuador, 240 m (QCAZ 14844, 20797-800); PROVINCIA SUCUMBIOS: La Selva lodge, $250 \mathrm{~m}$ (QCAZ 7408, 12093-95).

Osteocephalus taurinus. ECUADOR: PROVINCIA ORELLANA: Parque Nacional Yasuní, km 97 Pompeya-Iro road, 450 m (QCAZ 5301); Estación Científica Yasuní, Universidad Católica del Ecuador, 220 m (QCAZ 9007, 10604, 14804, 14954, 24449-50); PROVINCIA SUCUMBÍOS: Reserva de Producción Faunís- 
tica Cuyabeno, 220 m (QCAZ 5871-77); Puerto Bolívar, 240 m (QCAZ 27916, 27920); Zábalo, 220 m (QCAZ 27982, 28015); Chiritza-Puerto El Carmen road, bridge over Río Aguas Negras, 270 m (QCAZ 28485); Tarapoa-Puerto El Carmen road, bridge over Río Cuyabeno, 290 m (QCAZ 28435-36); PROVINCIA ZAMORA CHINCHIPE: Shaime, Nangaritza, 980 m (QCAZ 18230).

Osteocephalus verruciger. ECUADOR: PROVINCIA MORONA SANTIAGO: Nueve de Octubre (QCAZ 32266); Bosque Protector Abanico, Morona, 1647 m (EPNH 11444); Morona, 1530 m (EPN-H 11445); Río Sardinayacu, Palora, Parque Nacional Sangay, $1600 \mathrm{~m}$ (EPN-H 5940-42, 5947); PROVINCIA NAPO: near Santa Rosa de Quijos, 1661 m (QCAZ 45344); E of Volcán Sumaco, 1570 m (QCAZ 1560, 1562); Nueva Loja road between Cascabel 1 and 2, $1600 \mathrm{~m}$ (QCAZ 7783-84); Río Salado (QCAZ 17285); Sumaco, 1800-2100 m (QCAZ 8964); Pacto Sumaco (QCAZ 10907); km 13 Loreto-Coca road, 1324 m (QCAZ 22201); Río Hollín (QCAZ 1681, 2405); Coordillera de los Guacamayos, Cosanga-Archidona road, 1600 m (QCAZ 12206, 41108); El Reventador (QCAZ 29208); Cascada San Rafael, 1553 m (QCAZ 363, 13225,13247, 16954, 3203236); Cosanga, 339 m (QCAZ 15942); PROVINCIA SUCUMBIOS: Quito-Lago Agrio road, Río Azuela, 1680 m (QCAZ 15149, 15991-97, 16220, 16953, 22497 , EPN-H 6341, 11987, 12105-07, 12112, 12143); La Bonita (QCAZ 3175); Rosa Florida, 1185 m (QCAZ 20544); trail to Volcán Reventador, Gonzalo Pizarro, 1800 m (EPN-H 7052-53, 7059-60); PERU: REGIÓN DE AMAZONAS: Provincia Condorcanqui: Cabecera de la Quebrada Katerpiza, 1100 m (CORBIDI 09477); REGIÓN DE LORETO: Provincia Datem del Marañón: Cabecera de la Quebrada Wee, 1000 m (CORBIDI 09525).

Osteocephalus yasuni. ECUADOR: PROVINCIA SUCUMBIOS: Zábalo, $220 \mathrm{~m}$ (QCAZ 27998); Playas de Cuyabeno, 230 m (QCAZ 27816).

Trachycephalus jordani. ECUADOR: PROVINCIA EL ORO: Bosque Protector Puyango (QCAZ 35405).

Trachycephalus typhonius. ECUADOR: PROVINCIA PASTAZA: km 6 San RamónEl Triunfo road, Colonia Mariscal Sucre, trail to Río Pucayaku (QCAZ 38075). 